\title{
Safety Assessment of Graphene-Based Materials: Focus on Human Health and the Environment
}

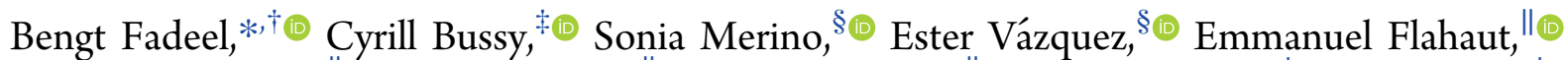

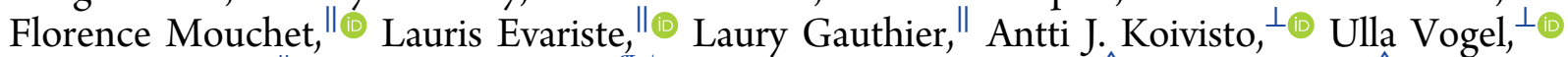

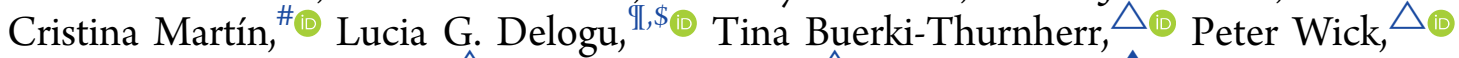

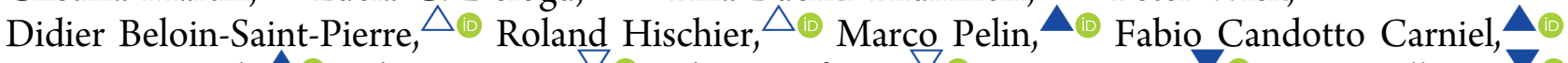

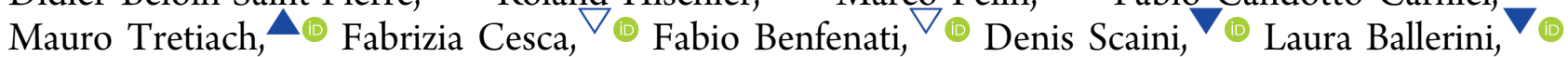
Kostas Kostarelos, ${ }^{\ddagger}$ Maurizio Prato, $*, \square, \square, \square$ and Alberto Bianco ${ }^{*}, \# \mathbb{C}$

${ }^{\dagger}$ Nanosafety \& Nanomedicine Laboratory, Institute of Environmental Medicine, Karolinska Institutet, 17777 Stockholm, Sweden

${ }^{\ddagger}$ Nanomedicine Laboratory, Faculty of Biology, Medicine \& Health, University of Manchester, Manchester M13 9PL,

United Kingdom

${ }^{\S}$ Faculty of Chemical Science and Technology, University of Castilla-La Mancha, 13071 Ciudad Real, Spain

"CNRS, Université Paul Sabatier, 31062 Toulouse, France

${ }^{\perp}$ National Research Centre for the Working Environment, 2100 Copenhagen, Denmark

${ }^{\#}$ University of Strasbourg, CNRS, Immunology, Immunopathology and Therapeutic Chemistry, 67000 Strasbourg, France

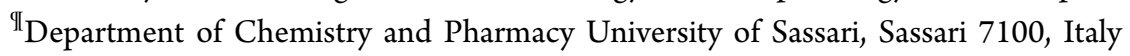

\$Istituto di Ricerca Pediatrica, Fondazione Città della Speranza, 35129 Padova, Italy

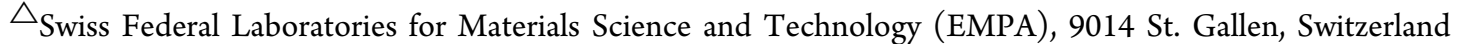

$\boldsymbol{\Delta}_{\text {Department of Life Sciences, University of Trieste, } 34127 \text { Trieste, Italy }}$

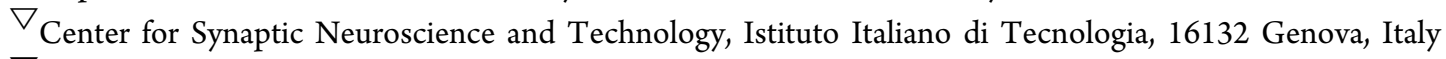

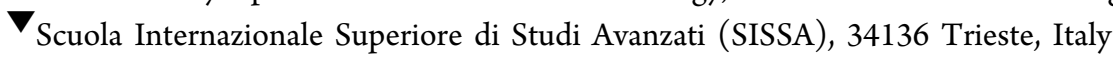

${ }^{\square}$ Department of Chemical and Pharmaceutical Sciences, University of Trieste, 34127 Trieste, Italy

- Carbon Nanobiotechnology Laboratory, CIC BiomaGUNE, 20009 San Sebastian, Spain

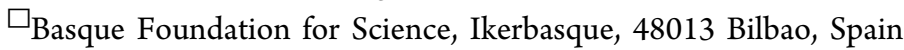

Supporting Information

ABSTRACT: Graphene and its derivatives are heralded as "miracle" materials with manifold applications in different sectors of society from electronics to energy storage to medicine. The increasing exploitation of graphene-based materials (GBMs) necessitates a comprehensive evaluation of the potential impact of these materials on human health and the environment. Here, we discuss synthesis and characterization of GBMs as well as human and environmental hazard assessment of GBMs using in vitro and in vivo model systems with the aim to understand the properties that underlie the biological effects of these materials; not all GBMs are alike, and it is essential that we disentangle the structure-activity relationships for this class of materials.

KEYWORDS: carbon nanomaterials, graphene, hazard, exposure, life cycle assessment, environment, safety, toxicity

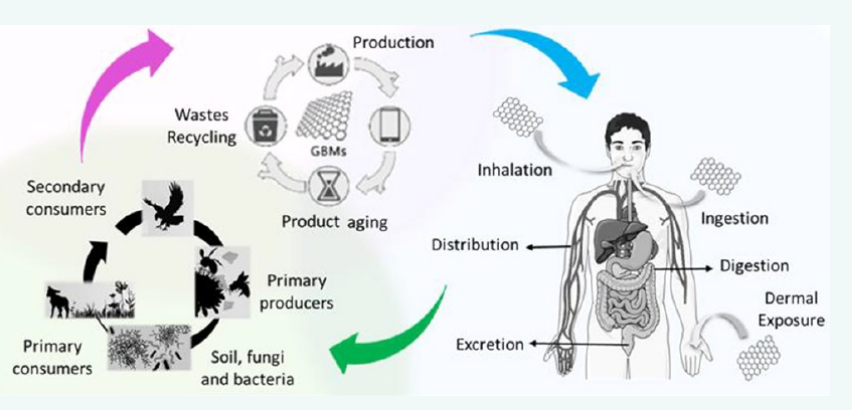

raphene is first-in-class-the first two-dimensional (2D) atomic crystal. ${ }^{1}$ The many extraordinary properties of this material, such as mechanical stiffness,
Received: June 22, 2018

Accepted: November 2, 2018

Published: November 2, 2018 
strength, and elasticity as well as the high electrical and thermal conductivity, have generated considerable excitement since the initial discovery, and graphene and its derivaties are currently being explored for a multitude of different applications. ${ }^{2}$ Safe and sustainable development of graphene-enabled technologies and products requires close attention to the potential impact of these materials on human health and the environment. ${ }^{3}$ Indeed, safety assessment is an integral part of the innovation process. $^{4}$

Material characterization, in turn, is a key element of hazard assessment. The toxicological evaluation of carbon nanotubes is a case in point. Ten years ago, carbon nanotubes were suggested to display "asbestos-like" pathogenicity in an animal model in the sense that long and rigid, but not short or tangled, carbon nanotubes elicited granuloma formation and inflammation following intraperitoneal injection in mice. ${ }^{5}$ We now understand that carbon nanotubes adhere to a certain extent to the so-called pathogenic fiber paradigm, and that some types of multiwalled carbon nanotubes may be considered as potentially carcinogenic to humans. ${ }^{6}$ However, other members of the same family of materials have been found to be nontoxic ${ }^{7,8}$ and may even undergo degradation, 9 suggesting that not all carbon nanotubes are asbestos-like (reviewed in ref 10). In fact, carbon nanotubes, if appropriately purified and surface-modified, hold promising prospects in nanomedicine, for instance, for drug or gene delivery and/or imaging. ${ }^{10}$

Thus, while it is evident that important lessons can be learned from previous studies of other engineered nanomaterials, it is equally important to avoid extrapolation from the study of one class of nanomaterials to another-if we acknowledge that new materials have new and useful properties, then we must also accept that such new materials could pose new or unanticipated risks. ${ }^{11}$ This is not to say that the biological or toxicological effects of a novel material are necessarily "novel". Indeed, the final common pathways of cellular or organ damage (e.g., oxidative stress, inflammation, carcinogenesis) may be conserved for different (nano)materials, ${ }^{12}$ but it is nevertheless of considerable importance to understand how those pathways are triggered: in essence, toxicology seeks to understand the structure-activity relationship of a chemical or a material. We need to understand the properties of the materials and how these are connected to the biological effects in order to make them both useful and safe. $^{13,14}$

The Graphene Flagship Project (www.graphene-flagship.eu) is, along with the Human Brain Project, the first of the European Commission's Future and Emerging Technology (FET) Flagship Projects, whose mission is to address major scientific and technological challenges through long-term, multidisciplinary research and development efforts. The Graphene Flagship was launched in 2013 and is expected to run for 10 years; the consortium consists of over 150 academic and industrial research groups in more than 20 countries. Safety assessment is an essential requirement that cannot be dissociated from the development of new technologies. Therefore, the Graphene Flagship has invested considerable efforts in evaluating the potential impact of graphene-based materials (GBMs) on human health and the environment. ${ }^{2}$ The aim of the present review is to provide a comprehensive view of human and environmental hazard assessment of GBMs, taking as a starting point the work that has been conducted during the first half of the Graphene Flagship, along with other relevant literature. We address the main exposure routes for GBMs and the key target organs including the immune system, the skin, the lungs, the cardiovascular system, the gastrointestinal system, the central nervous system, and the reproductive system, as well as a wide range of organisms including bacteria, algae, plants, invertebrates, and vertebrates in a variety of ecosystems. We also address the synthesis and characterization of GBMs as a thorough understanding of the material itself is crucial in any (nano)toxicological evaluation. ${ }^{15,16}$ We briefly discuss exposure and life cycle analysis of GBMs though the information is relatively sparse at this point. ${ }^{4}$ Overall, with this survey of recent research on the safety assessment of GBMs, we intend to emphasize the importance of knowing the material as "graphene" is not a single material but a class of materials, and it stands to reason that the biological effects of these materials may vary as a function of their intrinsic properties. ${ }^{17}$ Moreover, it is important to know the test system, as different tests may address different questions, but only within the applicability domain of the test. ${ }^{18}$ To understand the biological impact of GBMs, systematic studies using multiple test systems spanning from in vitro to in vivo models are required. Furthermore, close attention both to human health and the environment ${ }^{19,20}$ is needed in order to maximize the societal benefits of these novel materials.

\section{SYNTHESIS AND CHARACTERIZATION OF GRAPHENE-BASED MATERIALS}

One important concern in graphene research is that the term "graphene" is used in a generic manner to describe many different GBMs. ${ }^{17,21}$ In an attempt to remedy this situation, the Graphene Flagship proposed a classification scheme for GBMs that takes into account three key parameters: the number of graphene layers, the average lateral size, and the carbon-tooxygen $(\mathrm{C} / \mathrm{O})$ atomic ratio. ${ }^{22}$ The use of such a classification framework (Figure 1) (and see also Supporting Information) may facilitate the comparison between studies performed in different laboratories and may also enable the assignment of specific physicochemical properties with the safety profile of GBMs.

Synthesis of Graphene-Based Materials. There are numerous reports in the literature regarding different synthetic methodologies, ${ }^{23-25}$ but certain requirements need to be fulfilled when graphene materials are produced for biological applications including their toxicological evaluation. ${ }^{17}$ In general, for in vitro studies, the materials must be provided as stable aqueous solutions, and the amount of impurities should be carefully controlled. In addition to chemical contaminants arising from the synthesis, biological contaminants, i.e., microbes or parts of microbes (endotoxin), need to be considered, as production is often not performed under aseptic conditions or using sterile solvents. ${ }^{26}$ Some general approaches to obtain GBMs for biological applications are summarized below.

Graphene. Graphene dispersions can be produced via exfoliation of graphite using ultrasonication. ${ }^{27}$ For successful exfoliation in water, the assisted intercalation of small molecules or solvents between the layers is commonly used. For instance, insertion of chlorin-e ${ }_{6}(\mathrm{Ce} 6)$ showed a successful exfoliation of graphite in biocompatible media (water or phosphate buffer). ${ }^{28}$ Plant extracts have also been used for the exfoliation of graphite in water by sonication. ${ }^{29}$ Furthermore, the liquid phase exfoliation of graphite in different animal sera conducted to low toxicity graphene suspension using a regular kitchen blender. ${ }^{30}$ Using a different approach, Liu et al. devised an effective method to produce high-quality multilayer graphene in large amounts by the exfoliation of graphite under microwave irradiation through the intercalation of ammonium persulfate or hydrogen peroxide. ${ }^{31}$ 


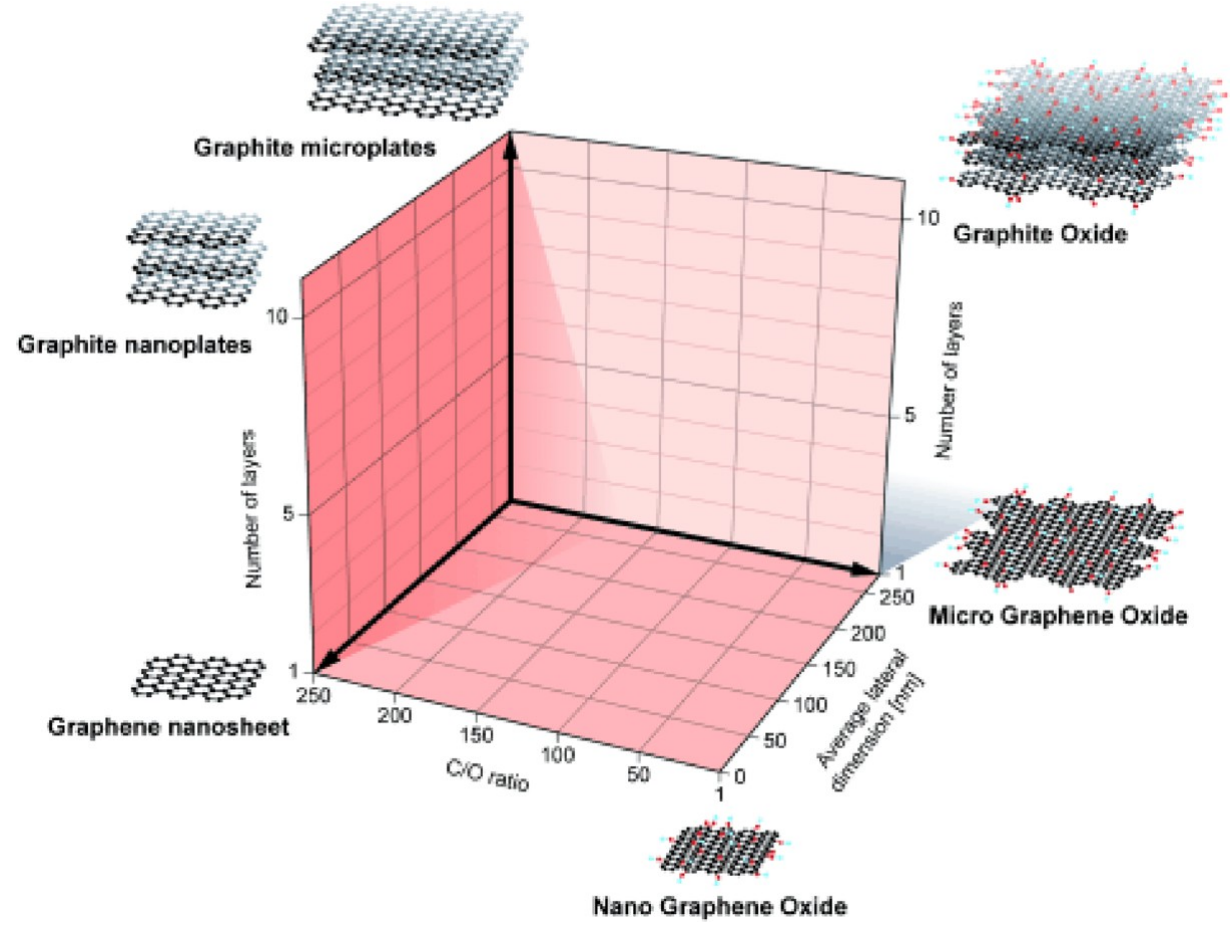

Figure 1. Classification framework for graphene-based materials. Reprinted with permission from ref 22. Copyright 2014 Wiley-VCH Verlag GmbH \& Co, KGaA, Weinheim.

The irradiation causes the decomposition of the oxidizing agents into gaseous oxygen, which gives rise to the exfoliation. Stable water dispersions of graphene can also be prepared by ball-milling treatments. In particular, the exfoliation of graphite through interactions with melamine allows the production of the material with low amount of defects. ${ }^{32}$ Excess melamine can subsequently be removed by dialysis. Moreover, after lyophilization of these aqueous graphene suspensions, a soft powder of few-layer (FLG) graphene is obtained, which can be easily dispersed in aqueous media. The process, developed in the Graphene Flagship, thus comprises four steps: (i) the mechanochemical intercalation of organic molecules (melamine) into graphite, followed by suspension in water; (ii) the washing of suspended graphene to eliminate most of the melamine; (iii) the isolation of stable graphene sheets; and (iv) freeze-drying to obtain graphene powder. ${ }^{33}$ Furthermore, a recent approach for scalable and environmentally friendly production of graphene via liquid phase exfoliation involved a wet ball-milling process in the presence of liquid nitrogen and several alcohols. ${ }^{34}$

Graphene Oxide (GO). Most of the methods proposed to synthesize GO are based on oxidation of graphite following a modified Hummers' protocol, which involves the use of oxidizing reagents and acids. However, this method yields $\mathrm{GO}$ with different degrees of oxidation and impurities. Additional purification steps are necessary to enhance the purity of the material. ${ }^{35}$ It has been reported that the starting graphitic materials play an important role not only in terms of general yields but also for the structural properties of the resulting GO sheets. ${ }^{36}$ Coleman et al. have recently prepared GO sheets with different sizes by sonication in aqueous dispersions and explored the relationship between the total sonication energy and the average size of the GO sheets. ${ }^{37}$ In order to avoid some of the pitfalls encountered with commercial preparations, GO suspensions were produced specifically in the Graphene Flagship from graphite flakes. These water-based suspensions were produced following a modified Hummers' method, ${ }^{35}$ further improved to ensure endotoxin-free suspensions of single- to few-layer GO sheets of high chemical purity. ${ }^{26,36}$ These materials now exist in a range of different lateral dimensions or thicknesses ${ }^{38,39}$ in order to assess the role of these physicochemical characteristics with respect to biological impact. ${ }^{40,41}$ Furthermore, a suite of characterization techniques has been established in order to confirm the quality, reproducibility, and low batch-to-batch variability of each synthesis. ${ }^{42}$

Reduced Graphene Oxide ( $r G O$ ). Typical methods to obtain rGO include the chemical, thermal, and electro/photochemical reduction of GO. Chemical reduction prevails over nonchemical reduction approaches because of the improved quality, efficiency, and the fact that stable dispersions of rGO can be obtained. The most effective chemical reductant is hydrazine; however, this reagent is not very popular because of its toxicity to humans and the environment. During the past decade, hydrazine and other toxic chemicals have been replaced by more biocompatible and environmentally friendly reductants, known as "green" reducing agents. ${ }^{43}$ Some examples include vitamin C, ${ }^{44}$ starch-based materials, ${ }^{29}$ sugars, ${ }^{45}$ plant extracts, ${ }^{46}$ or microorganisms. ${ }^{47}$ Nevertheless, even these reductants have shortcomings related to purification processes and the difficulty of large-scale production. ${ }^{48}$ In a recent study, GO was reduced to rGO by ultrasonic irradiation at $50{ }^{\circ} \mathrm{C}$ in the absence of a reducing agent. ${ }^{49}$ It is often shown that a simple heat treatment is enough to perform the reduction of GO even in air. ${ }^{50}$ This is attractive because it is fast, and one avoids contamination of the material with exogenous elements (such as nitrogen when hydrazine is used). When heating is performed in a protective atmosphere (vacuum, inert gas), a rather high $\mathrm{C} / \mathrm{O}$ ratio is obtained. ${ }^{51}$

Although most of the synthetic methodologies discussed above yield high-quality graphene, the impossibility of scaling these methods limits their industrial applications. Therefore, obtaining a large amount of biocompatible graphene in a simple and low-cost manner remains a considerable challenge. Methods 
such as the liquid phase exfoliation of graphite, ${ }^{52,53}$ including "green" reducing reagents (e.g., honey), ${ }^{44}$ or exfoliating agents (e.g., sucrose, glucose, or silk proteins), ${ }^{55-57}$ and techniques such as microwave ${ }^{58}$ or ball-milling ${ }^{59,60}$ are the most recent approaches employed to increase the concentration of graphene dispersions in an economically feasible and environmentally friendly way. Recently, some companies have developed the electrochemical exfoliation of graphite. ${ }^{61}$ This method has great potential; however, the quality of graphene is still low as defects are generated during the process. ${ }^{61} \mathrm{GO}$ can be more easily produced, and companies are already able to produce hundreds of kilograms of GO.

Characterization of Graphene-Based Materials. For proper hazard assessment, the materials need to be well-characterized using standardized and validated characterization techniques. ${ }^{62}$ Given the variety of available GBMs, a description of the physicochemical properties must be provided in all toxicological and pharmacological studies. ${ }^{63}$ For chemical characterization, the most commonly used techniques are X-ray photoelectron spectroscopy (XPS), Fourier transform infrared (FTIR) spectroscopy, Raman spectroscopy, X-ray diffraction (XRD), thermogravimetric analysis (TGA), and elemental analysis. Transmission electron microscopy (TEM), scanning electron microscopy (SEM), and atomic force microscopy (AFM) provide information on the morphology and dimensions of the material (Table 1). The limulus amoebocyte lysate (LAL) assay

Table 1. Characterization of Graphene-Based Materials

\begin{tabular}{|c|c|}
\hline property & technique \\
\hline lateral dimensions & $\begin{array}{l}\text { electron microscopy (TEM, SEM) } \\
\text { atomic force microscopy (AFM) } \\
\text { dynamic light scattering (DLS) }\end{array}$ \\
\hline number of layers & $\begin{array}{l}\text { electron microscopy (TEM) } \\
\text { atomic force microscopy (AFM) } \\
\text { Raman spectroscopy }\end{array}$ \\
\hline surface charge & $\zeta$-potential \\
\hline $\mathrm{C} / \mathrm{O}$ atomic ratio & $\begin{array}{l}\text { X-ray photoelectron spectroscopy (XPS) } \\
\text { elemental analysis }\end{array}$ \\
\hline $\begin{array}{l}\text { chemical structure/ } \\
\text { functionalization }\end{array}$ & $\begin{array}{l}\text { X-ray photoelectron spectroscopy (XPS) } \\
\text { elemental analysis } \\
\text { Raman spectroscopy } \\
\text { thermogravimetric analysis (TGA) } \\
\zeta \text {-potential } \\
\text { Fourier transform infrared spectroscopy } \\
\text { (FTIR) }\end{array}$ \\
\hline metal impurities & $\begin{array}{l}\text { X-ray electron diffraction (XRD) } \\
\text { total reflection X-ray fluorescence (TXRF) } \\
\text { atomic adsorption spectroscopy } \\
\text { inductively coupled plasma-mass spectrometry } \\
\text { (ICP-MS) }\end{array}$ \\
\hline endotoxin content & $\begin{array}{l}\text { limulus amoebocyte lysate (LAL) assay } \\
\text { macrophage-based TNF expression test } \\
(\text { TET) }\end{array}$ \\
\hline
\end{tabular}

is commonly used to check for endotoxin content of nano- and biomaterials, but recent studies have shown that GBMs cause interferences with the assay, and an alternative macrophagebased assay was proposed. ${ }^{26}$ Endotoxin contamination may mask or lead to the misinterpretation of the biological effects of biomaterials and precludes their medical use. ${ }^{64}$

It is important to note that even when using a synthetic methodology that selectively leads to a certain graphene material (e.g., GO) the final product is often not homogeneous, but a broad distribution of components with different properties. Hence, whereas apparently contradictory results have appeared in the literature, for example, in terms of bioaccumulation in different organs (discussed below), it is important to point out that these disparities may be due to the existence of different graphene morphologies as well as the use of different biological model systems. In general, the toxicity (or safety) of GBMs depends on physicochemical properties such as size, number of layers, and surface chemistry ${ }^{22}$ (supporting text and Figure S1). Moreover, the presence of impurities and the graphene synthetic methodology used may also influence the toxicological responses. ${ }^{65}$ The lateral dimension of the material is one important parameter, as we shall discuss below. The number of graphene layers is also important as this will determine specific surface area, absorptive capacity, and bending stiffness. As the surface area is inversely proportional to the number of layers, it is expected that the adsorptive capacity for biological molecules (biocorona formation $)^{10}$ increases as this number decreases. GBMs may possess a wide variety of chemical surfaces. Hence, the surface of pristine graphene is hydrophobic, and the surface of GO with extensive oxygenated functions like carboxyl, epoxy, and hydroxyl groups is highly hydrophilic, whereas rGO presents intermediate characteristics. The different degree of oxidation $(\mathrm{C} / \mathrm{O}$ ratio) may determine the interactions with proteins and other biomolecules. ${ }^{66}$ Furthermore, functionalization with poly(ethylene glycol) (PEG), poly(vinyl alcohol), chitosan, or pluronic modulates the biocompatibility of GBMs. ${ }^{67-69}$ Functionalization can affect the surface charge of the material, which may impact cellular internalization and other biological interactions. In conclusion, the way in which GBMs are prepared is of key importance for the potential impact on biological systems. Furthermore, for specific applications, quality and adherence to regulatory standards will be critical. For such applications, heterogeneity in the produced materials (batch-to-batch variability for a single producer or variability between different producers) and lack of international and regulatory standards are important issues, perhaps not for their initial translation to the market but for a wider acceptance and penetration of the market. Moreover, in the context of clinical translation of GBMs, one of the issues that one will have to overcome is the current medium-to-high variability observed between different synthesis batches. Production methods will have to progress toward better GMP (good manufacturing practices) compliance (e.g., narrow polydispersity, homogeneous functionalization) to allow for approval and registration of GBMs.

Dissecting the Role of Material Properties: Reference Libraries. To dissect the role of (nano)material properties on the biological impact, access to appropriate reference material libraries should be considered. In 2009, $\mathrm{Nel}$ and co-workers proposed the creation of a standard nanomaterial library including the principal classes of nanomaterials and nanoparticles. ${ }^{70}$ The authors stated "it is important to link the library development to a nanomaterial classification that allows toxicological mechanisms to be interpreted in terms of intrinsic material properties". Since then, some examples of such material libraries have emerged. Hence, a custom-designed material library for testing the toxicity of metal oxides was developed taking into account well-established methods available to produce and characterize such nanoparticles. ${ }^{71}$ Walkey et al. ${ }^{72}$ characterized the serum protein corona "fingerprint" formed on a library of 105 surface-modified gold nanoparticles, thereby providing a rich source of information with which to develop quantitative relationships to predict the biological responses to such 
nanoparticles. Zhou et al., ${ }^{73}$ in turn, developed a combinatorial library of 80 functionalized carbon nanotubes to reveal structure-activity relationships with respect to cytotoxicity and immune responses. A systematic methodology relying on the combination of computational methods and the properties of nanomaterials to generate libraries that permit rapid screening of cells and biologically relevant organisms has been recently proposed. $^{74}$ The authors offered examples of libraries that can be constituted of nanomaterials and nanoparticles characterized by their chemical composition. Graphene was included in the family of nanocarbons along with carbon nanotubes and fullerenes. However, we believe that the chemical composition is not the only parameter that one should consider as the chemical structure of graphene differs remarkably from that of nanotubes and fullerenes. Furthermore, graphene constitutes a whole class of materials with different characteristics and, therefore, likely also different biological effects. ${ }^{22}$ Our recent attempts to classify GBMs according to certain well-defined parameters ${ }^{21,22}$ are important first steps toward the development of a reference library including graphene and its derivatives, enabling a detailed dissection of structure-activity relationships to assess biological effects. It is noted that GBMs have not yet been included, for instance, in the nanomaterial repository of the European Commission's Joint Research Centre (JRC), and this is likely related to the fact that the International Organisation for Standardisation (ISO) is still deliberating on the definition and material specifications of graphene and related materials. The inclusion of graphene as a representative industrial nanomaterial in the aforementioned repository and the creation of a specific reference library of wellcharacterized GBMs will be fundamental for benchmarking purposes in basic and regulatory research. However, in this moment, we need to rely mainly on the physicochemical characteristics of individual GBMs that are reported in the literature.

\section{BIODISTRIBUTION AND FATE OF GRAPHENE-BASED MATERIALS}

The fate of GBMs in the body of an exposed organism is governed both by their intrinsic physicochemical characteristics such as lateral dimensions, thickness, and $\mathrm{C} / \mathrm{O}$ ratio/ functionalization and by their extrinsic or acquired features upon contact with the biological environment, largely dictated by the biocorona. ${ }^{10}$ In addition, the portal of entry into the body is also a major determinant of the subsequent fate of the materials. The intrinsic characteristics of GBMs are expected to influence their biodistribution, translocation to secondary organs, accumulation, degradation, and clearance. However, these characteristics may be altered by the adsorption of proteins and other biomolecules present in the biological milieu. ${ }^{75}$ In addition, the local ion concentration may affect nanomaterials including GBMs. ${ }^{76}$ These interactions can modify the GBMs in such a way that the initial shape, surface charge, thickness (in turn affecting the hydrodynamic diameter), or colloidal stability is altered, which may impact biological behavior of the material. ${ }^{77}$ Notably, these acquired features may change dynamically over time and evolve as a function of the local environment as GBMs move from one biological compartment to another (for instance, from the lungs to the bloodstream). However, the intrinsic material properties are also subject to change, as a result of degradation by immune cells (discussed below) or other forms of biotransformation, ${ }^{78}$ thus underscoring the importance of characterizing the material not only in its pristine form but also in situ (in the test system during or after exposure) to the extent that this is possible. With this in mind, we discuss studies on the biodistribution and fate of GBMs upon various routes of administration relevant either for occupational or environmental exposures or routes that are relevant for biomedical applications of GBMs.

Multiple Routes of Exposure to Graphene-Based Materials. To investigate the impact of oral administration, Zhang et al. labeled small and large (both nanosized) rGO sheets with ${ }^{125}$ I to assess their biodistribution over 60 days after oral gavage. ${ }^{79}$ Both materials were found in blood, heart, lungs, liver, and kidneys, with a significantly higher amount in kidneys when compared to the control at day 1 , decreasing rapidly, but still remaining above control by days 15 and 60 . These results suggest that both materials were quickly absorbed in the gastrointestinal tract and reached secondary organs via the systemic circulation. In contrast, different outcomes were reported in a study on PEGylated GBMs. ${ }^{80}$ In the latter case, the in vivo biodistribution after oral gavage of PEGylated, nanosized GO, large rGO, and nanosized rGO was investigated using ${ }^{125} \mathrm{I}$ labeling. Radioactivity was detected in the stomach and intestine but not in any other major organs at $4 \mathrm{~h}$. The signal was no longer detectable at $24 \mathrm{~h}$, suggesting no intestinal adsorption of PEGylated GO. In line with these findings, it was demonstrated that GO (small and large lateral dimensions) does not penetrate across a fully differentiated enterocyte-like Caco- 2 cell monolayer. ${ }^{81}$

The inhalation route is of key relevance for human exposure. Li et al. studied nanosized GO (lateral dimension: $10-800 \mathrm{~nm}$, 1-2 layer) labeled with ${ }^{125} \mathrm{I}$ to test the biodistribution after intratracheal instillation. ${ }^{82}$ The vast majority of the GO sheets was found in the lungs, decreasing progressively from $10 \mathrm{~min}$ to $12 \mathrm{~h}$. Minor amounts were also detected in the blood, liver, and kidneys. These results could be ascribed to translocation to the blood either directly from the lungs or via intestinal adsorption as large amounts of the material were also detected in the stomach and intestines, potentially due to mucocilliary clearance, swallowing, and redistribution to the gastrointestinal tract. Macroscopic observation of the blackness of the lungs revealed that materials were long-lasting (black regions found for up to 3 months). However, there was a clear decrease of the blackness from day 1 to 90 , suggesting clearance from the lungs. Using the same route of administration, few-layer graphene platelets labeled with ${ }^{14} \mathrm{C}$ (lateral dimension: $60-590 \mathrm{~nm} ; 1-4 \mathrm{~nm}$; 4-6 layers, C/O ratio: 14.8 ) were tracked up to 28 days in mice. ${ }^{83}$ The material was found primarily in the lungs but also in the stomach and intestines in much lower amounts, suggesting a mucocilliary clearance mechanism followed by swallowing of the inhaled materials. The authors noted a time-dependent decrease in all investigated organs and a negligible amount of material in the liver and spleen, suggesting a very limited translocation to the bloodstream. The observed biodistribution of the graphene platelets is reminiscent of the biodistribution of ${ }^{14} \mathrm{C}$-labeled multiwalled carbon nanotubes following pharyngeal aspiration, with accumulation in the spleen of mice. ${ }^{84}$

The impact of subcutanous administration of PBS-based suspensions of GO (C/O ratio: 2.8) was compared to that of less oxidized GO (GO-R) (C/O ratio: 3.1 ) prepared by using less potassium permanganate during the modified Hummers' method. ${ }^{85}$ No trace metal impurities were detected in the samples. Unlike GO, there was evidence of early monocyte recruitment at the interface between GO-R and the subcutaneous tissue and also infiltration of monocytes within the GO-R macrostructure at day 3. At days 7 and 14, some infiltration of the GO macrostructure by macrophages and fibroblasts was noted, whereas these cells had completely infiltrated GO-R, with material-laden 
macrophages evidenced in both conditions. ${ }^{85}$ At day 14 , there was also early signs of collagen deposition (fibrosis) for GO-R. By day 30 , the GO macrostructure was fully infiltrated by macrophages, fibroblasts, and giant cells as expected for a typical foreign body reaction, whereas the GO-R macrostructure showed more advanced signs of healing, tissue repair processes, and extracellular matrix remodeling but without fibrosis. The authors suggested that the absence of fibrosis could be explained by the combination of macrophage uptake of the materials, a mild inflammatory response at the edges of the macrostructure, and indications of clearance from the site of injection. ${ }^{85}$

Following repeated intraperitoneal (i.p.) injections, graphite (average size: $3-4 \mathrm{~nm}$ ) and GO (average size: $8-25 \mathrm{~nm}$ ) powders resuspended in saline solution without surfactant were found to form macroscopic aggregates of up to $2 \mathrm{~mm}$ in the peritoneal cavity. ${ }^{86}$ These materials accumulated at the site of injection but also randomly throughout the peritoneal cavity without any sign of clearance or toxicity to the organs or the blood compartment. Furthermore, in order to assess the influence of GO oxidation degree (i.e., C/O ratio) on biodistribution after i.p. administration, PBS suspensions of GO were compared to those of GO-R. ${ }^{85}$ An increase in monocytes recruited to the peritoneal cavity was noticed at day 3 persisting over 2 weeks for GO in comparison to the less oxidized GO-R. Cells retrieved from the peritoneal cavity of treated animals and cultured for $12 \mathrm{~h}$ in vitro were more prone to secrete pro-inflammatory cytokines and chemokines in GO compared to cells retrieved from animals exposed to GO-R. In addition, GO-R seemed to be cleared more rapidly than GO, suggesting that persistence/clearance rate may be linked to monocytic cell recruitment and inflammogenicity of these materials. ${ }^{85}$

To study the biodistribution after i.p. administration of biomedically relevant materials, different types of GBMs were prepared from GO (lateral dimension: $300-700 \mathrm{~nm}$ ) to create PEGylated forms of nanoGO (lateral dimensions: $10-40 \mathrm{~nm}$ ) or rGO (lateral dimensions: $50-80 \mathrm{~nm}$ ) and nano-rGO (lateral dimensions: $10-30 \mathrm{~nm}$ ), and the materials were labeled with ${ }^{125} \mathrm{I} .{ }^{80}$ After 1 day, all the materials were accumulating primarily in the liver and spleen of exposed mice. However, after 7 days, while the nanoforms (nGO-PEG and nRGO-PEG) slightly decreased in the liver and slightly increased in the spleen, the larger-sized form (RGO-PEG) increased dramatically from day 1 to day 7 in these two organs. Interestingly, upon i.p. injection and unlike the PEGylated derivatives, nonPEGylated GO was found to form aggregates in the peritoneal cavity. However, this result was only based on macroscopic observation, as the authors did not perform radioactivity-based biodistribution analyses for the non-PEGylated materials. Black materials thought to be the injected materials were found in the histological sections up to 30 days postinjection. In another study, PEGylated rGO (lateral dimension: $\sim 1 \mu \mathrm{m}$, thickness: 4-9 $\mathrm{nm}, \mathrm{C} / \mathrm{O}$ ratio: 3.7 ) were prepared from graphite flakes following the Hummers' method to study their biodistribution, clearance, and toxicological profile in mice upon i.p. administration. ${ }^{87}$ Raman spectroscopy complemented by cluster analysis was used to map the distribution and revealed the presence of the PEGylated structures in brain, kidney, liver, and spleen. Following i.p. injection, most materials were found in the spleen after 3 days. However, whereas material content decreased over time in the spleen, it increased in the brain at 7 and 14 days, decreasing by day 21 , and increased dramatically in the liver by day 21 . The authors suggested that the materials crossed the blood-brain barrier, but direct evidence to support this notion was not presented. ${ }^{87}$
Bridging Nanosafety and Medicine: Intravenous Administration. One of the most common routes of administration for biomedical applications of nanomaterials is the intravenous (i.v.) route. Considerable research and development efforts in nanomedicine have established that shape, size, and surface charge are the most important physicochemical parameters determining biodistribution and fate of a nanomaterial after i.v. injection. ${ }^{88}$ Several studies have been published on the biodistribution of GBMs following i.v. injection. Qu et al. studied i.v. injection of GO suspended in PBS or GO dispersed in $1 \%$ Tween 80 -PBS and noted a higher accumulation in lungs for GO-PBS (lateral dimension: $300-1000 \mathrm{~nm}$ ). ${ }^{89}$ In contrast, accumulation in the liver was higher for GO-PBS-Tween 80 when compared to that for GO-PBS. Even though these conclusions were based only on the blackness of organs following treatment and observation of brown/black matter in histological sections, the results support the idea that better colloidal stability helps the GO sheets to pass through lung capillaries more easily. GO sheets functionalized with poly(sodium 4-styrenesulfonate) (lateral dimension: $300-700 \mathrm{~nm}$, thickness: $1-4 \mathrm{~nm}$ ) and labeled with the fluorescent $\mathrm{Cy} 7$ dye were used to assess biodistribution using whole-body live imaging. ${ }^{90}$ When organs were harvested at $24 \mathrm{~h}$, fluorescence was found only in the liver and bladder. Fourteen days after injection, there was obvious macroscopic presence of materials in the lungs, liver, and spleen, which all appeared black in comparison to corresponding organs from PBS-treated animals. Materials were still present in these organs after 180 days, as evidenced by black matter in histological sections. In a recent study, nonlabeled PEGylated rGO (lateral dimension: $\sim 1 \mu \mathrm{m}$, thickness: $4-9 \mathrm{~nm}, \mathrm{C} / \mathrm{O}$ ratio: 3.7$)$ was evaluated for its biodistribution after i.v. injection using Raman spectroscopy. ${ }^{87}$ Most materials were found in the liver and spleen at the earliest time point of 3 days, in agreement with other studies, but transiently increased in the brain at days 7 and 14 days before decreasing by day 21 . The biodistribution and degradation of oxidized FLG platelets (lateral dimension: $150-220 \mathrm{~nm}$ ) were studied over 90 days using Raman confocal imaging. ${ }^{91}$ The authors reported that the materials (dispersed in PBS) agglomerated and formed macrostructures of $0.5-10 \mu \mathrm{m}$ in the lungs, liver, kidneys, and spleen. The aggregates were still present after 90 days despite some signs of degradation at the material edges.

Yang et al. reported quantitative evaluation of the biodistribution of GO after i.v. administration using ${ }^{125}$ I-labeled nanosized GO further functionalized with PEG (lateral dimension: 5-50 nm, thickness: $1-2 \mathrm{~nm}) .{ }^{67}$ Biodistribution was followed over 60 days, revealing a higher accumulation in the spleen compared to that in the liver at all time points and a gradual decrease of the amount of materials in these reticuloendothelial system organs. The decrease could be explained by a continuous clearance as radioactivity was detected in both urine and feces over the 60 day period, with a higher amount in urine compared to feces, suggesting glomerular filtration of the material. In a related study, the biodistribution of nanosized GO functionalized with PEG was compared to the distribution of nanosized GO not functionalized with PEG. ${ }^{92}$ Labeling with ${ }^{125} \mathrm{I}$ was used to track and quantify the two materials. Both materials accumulated in the lungs without evidence of clearance during the tested period (from $10 \mathrm{~min}$ to $6 \mathrm{~h}$ ). Although a very small amount of the two materials could be found in the spleen, there was an extensive accumulation of both materials in the liver that decreased with time. The non-PEGylated GO accumulated twice as much in the liver when compared to PEGylated GO. Three months after injection, black matter was still found in 
both lungs and liver, with signs of tissue injury and remodeling, evidenced by collagen deposition (fibrosis). Hence, whereas PEGylation reduced the accumulation in reticuloendothelial system organs by extending the blood circulation, this only marginally alleviated the adverse outcomes upon accumulation of $\mathrm{GO}$ in these organs. ${ }^{92}$

Sasidharan et al. prepared FLG (lateral dimension: $100-200 \mathrm{~nm}$, thickness: $0.8 \mathrm{~nm}$ ) and two derivatives thereof (carboxylated FLG-COOH and PEGylated FLG-PEG) covalently labeled with the radioactive marker ${ }^{99} \mathrm{Tc}$ to study their biodistribution over $24 \mathrm{~h} .{ }^{93}$ FLG-COOH showed higher accumulation in the lungs over the $24 \mathrm{~h}$ period, whereas FLG-PEG accumulated initially in the lungs, but by $24 \mathrm{~h}$, the material had relocated to liver and spleen. Using histological sections, the authors confirmed these results, showing that FLG and FLG-COOH accumulated in lungs for a long period of time (up to 90 days) causing damage, whereas FLG-PEG gradually exited the lungs (no sign at 90 days) and did not cause adverse effects in the lungs. All three materials were also found in spleen, liver, and kidneys for periods of up to 90 days, and signs of tissue damage were noted for FLG and FLG-COOH but not for FLG-PEG. ${ }^{93}$ In another study, small (lateral dimension: 148-160 nm, 1-2 layers, $\mathrm{C} / \mathrm{O}$ ratio: 2.28) and large (lateral dimension: 556-780 nm, 1-2 layers, C/O ratio: 2.70) GO sheets labeled with ${ }^{125}$ I were injected i.v. in mice to study their biodistribution over 180 min..$^{94}$ A longer blood circulation time was observed for small GO when compared to large GO. Conversely, small GO sheets accumulated mainly in the liver (peaking at 5 min then progressively decreasing by $180 \mathrm{~min}$ ) with only a limited amount found in the lungs and spleen (rapidly disappearing from these organs). In contrast, large GO sheets were mostly detected in the lungs, with only a marginal decrease over the 180 min period. A small amount of large GO was also found in the liver. Finally, when increasing the dose of injected small GO by a factor of 10 , the authors reported that organ accumulation of GO clearly shifted from the liver to the lungs, suggesting congestion in the capillary bed of the lungs possibly due to the formation of agglomerates of GO sheets. ${ }^{94}$ These two comparisons (i.e., large versus small GO and low versus high concentration of small GO) both support the idea that larger plate-like structures or agglomerates of smaller plate-like structures are more likely to be trapped in the lungs following i.v. injection, possibly by congestion of the blood vessels.

Finally, a series of investigations using radiolabeled GO sheets functionalized with dioctyltin diacetate (DOTA) to chelate the radiolabel were executed in the Graphene Flagship (Figure 2). Single- to few-layer GO sheets (lateral dimension: 100-400 nm with DOTA, thickness: $2-10 \mathrm{~nm}$ ) were found to accumulate in both liver and spleen, with a decrease of radioactivity from 1 to $24 \mathrm{~h}$ in the liver and an increase in the spleen. ${ }^{95}$ Whereas the amount in the liver was, on average, higher than that in the spleen at $1 \mathrm{~h}$, it was higher in the spleen compared to the liver at $24 \mathrm{~h}$. Furthermore, biodistribution of these thin GO sheets (thickness: $1-4 \mathrm{~nm}$ without DOTA; $1-10 \mathrm{~nm}$ with DOTA) was compared to thick GO sheets (5-30 nm with DOTA) obtained by restacking of the thin GO sheets due to aging. ${ }^{40}$ Thicker materials accumulated to a greater extent than the thin materials in both liver and spleen over the first hour after injection. Importantly, in both studies, a large amount of radioactivity was observed in the bladder at early time points. These results suggested that a large amount of i.v.-injected GO sheets had undergone renal glomerular filtration, as confirmed by the transient presence of radiolabeled GO sheets in kidneys and the detection of GO sheets in the urine, verified by TEM and Raman spectroscopy. ${ }^{40}$ The impact of this extensive renal clearance of GO sheets on kidney physiology was investigated in a subsequent study. ${ }^{41}$ No sign of nephrotoxicity or glomerular barrier dysfunction was identified despite the translocation of GO sheets from the blood to the urine. One may assume that the plate-like GO sheets would need to transiently adopt a different shape (e.g., through folding, crumpling, or wrinkling) in order to pass through the endothelial fenestra of the glomerular filtration barrier in the kidney.

Collectively, these studies have shed light on the biodistribution and fate of some GBMs following various administration routes. Overall, there is evidence that various GBMs are able to cross physiological barriers, reaching secondary organs distant from the point of entry. However, due to the scarcity of the published data and lack of systematic investigations, it is still too early to reach definitive conclusions with respect to relationships between physicochemical features and the biodistribution patterns of GBMs. Moreover, the long-term fate of GBMs at the site of accumulation is also important. It is acknowledged that the generation of such data is, however, far from trivial and requires labeled materials that can be tracked and quantitatively measured over long periods of time, even as GBMs undergo transformation or biodegradation in the body.

\section{HAZARD ASSESSMENT OF GRAPHENE-BASED MATERIALS}

Nanomaterials can enter into the body through different routes, and inhalation, dermal adsorption, and ingestion are the most likely routes of unintended exposure in occupational or environmental settings. ${ }^{96,97}$ Dermal injection is pertinent in the particular case of tattoo pigments, some of which are nanosized. ${ }^{98}$ Parenteral administration and primarily intravenous injection is relevant for intentional exposure to nanomaterials designed for specific medical applications. Nanomaterials may subsequently travel through the body and reach sites beyond their initial portal of entry, as discussed above. However, at some point, the materials manifest their biological (or toxicological) effects at the level of specific target organs. ${ }^{99}$ The potential adverse outcomes of nanomaterial exposure have been extensively investigated over the past decade, with particular attention to common classes of nanomaterials including metal and metal oxide nanoparticles and carbon-based materials, especially carbon nanotubes. These studies have informed us on specific material features that contribute to toxicity, for instance, particle dissolution and the release of toxic metal ions in the case of certain metal nanoparticles, or the high aspect ratio and fiber-like dimensions in the case of long and rigid carbon nanotubes. ${ }^{6}$ Hazard assessment of GBMs has been lagging behind, but in the past 5 years or more, the potential toxicity of GBMs has been explored in a systematic fashion both in vitro and in vivo, in the EU-funded Graphene Flagship project and elsewhere. The following sections provide an overview of the toxicological impact of GBMs on the immune system, our primary defense against foreign intrusion, as well as the pulmonary, dermal, cardiovascular, gastrointestinal, reproductive, and central nervous systems, following which we will discuss the environmental impact of GBMs.

Immune Interactions of Graphene-Based Materials. The immune system consists of complex molecular and cellular networks that protect our body from infections and other exogenous materials while maintaining tolerance to self-components. In the development of new materials, it is fundamental to assess their impact on the immune system in order to 
A
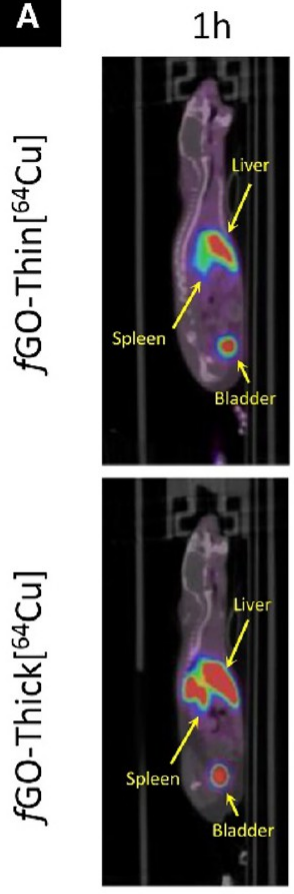

C 1h

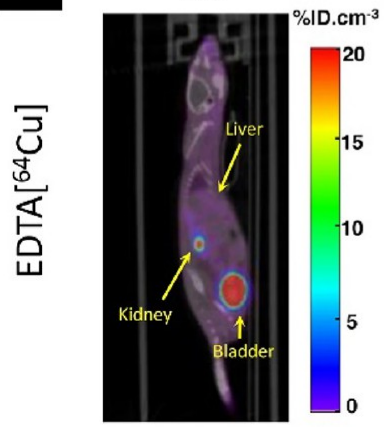

3.5h
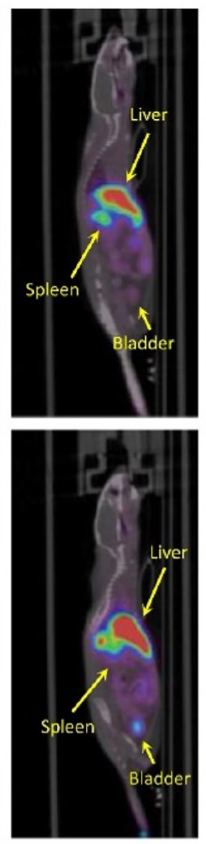

$24 \mathrm{~h}$

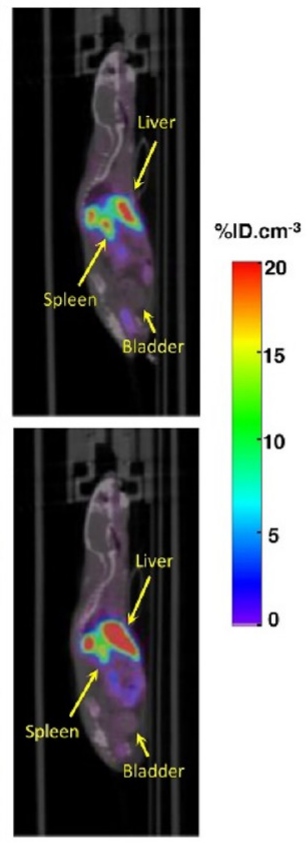

B
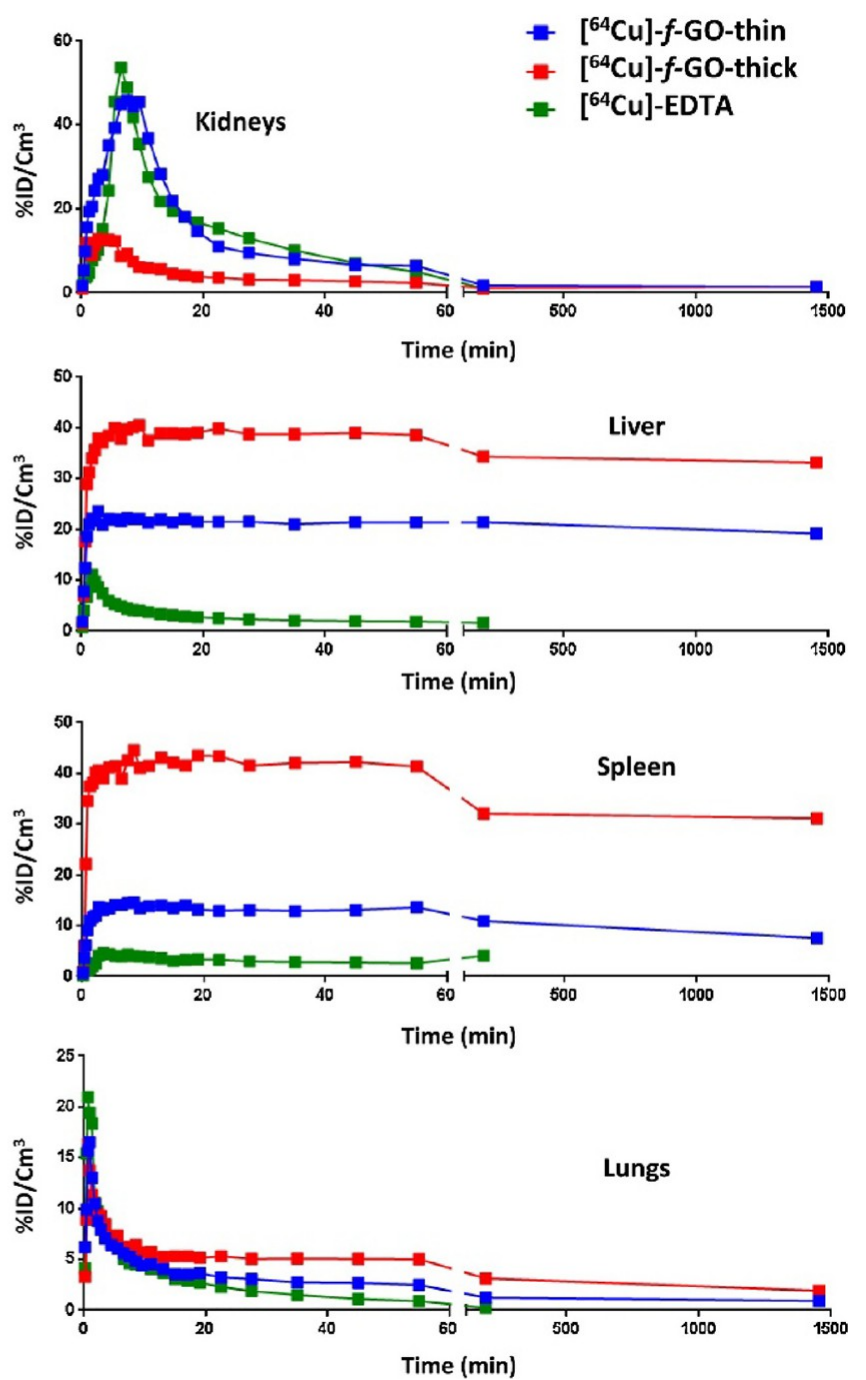

Figure 2. Biodistribution of GO. PET/CT imaging and tissue distribution of $\left[{ }^{64} \mathrm{Cu}\right]-f$-GO-thin, $\left[{ }^{64} \mathrm{Cu}\right]-f$-GO-thick, and $\left[{ }^{64} \mathrm{Cu}\right]-\mathrm{EDTA}$. (A) Whole-body PET/CT images of C57BL/6 mice injected intravenously with $\left[{ }^{64} \mathrm{Cu}\right]-f$-GO-thin (top) and $\left[{ }^{64} \mathrm{Cu}\right]-f$-GO-thick (bottom) at different time points $(1,3.5,24 \mathrm{~h})$. (B) Time-activity curves of major organs of C57BL/6 mice injected with $\left[{ }^{64} \mathrm{Cu}\right]-f-\mathrm{GO}-$ thin, $\left[{ }^{64} \mathrm{Cu}\right]-f-\mathrm{GO}$ thick, and control $\left[{ }^{64} \mathrm{Cu}\right]$-EDTA. (C) Whole-body PET/CT images of a C 57BL/6 mouse injected intravenously with the control sample $\left[{ }^{64} \mathrm{Cu}\right]-$ EDTA, showing almost complete excretion and no tissue accumulation after $3 \mathrm{~h}$. Reprinted with permission from ref 40 . Copyright 2016 Elsevier.

understand if the presence of such materials can be tackled, eventually leading to their elimination, or to clarify if the persistence of the materials provokes chronic diseases. ${ }^{100}$ Macrophages are key cells of the innate immune system tasked with eliminating exogenous and endogenous materials. Therefore, it is important to know if GBMs affect the viability and/or activation of macrophages. ${ }^{101}$ One of the first studies to address this question compared the effect of GO of different sizes on human and murine primary macrophages. ${ }^{102}$ The three GO materials tested displayed a dose-dependent cytotoxic effect. GO of smaller lateral size (130 and $270 \mathrm{~nm}$ ) were internalized to a higher extent in comparison to large GO $(\sim 1320 \mathrm{~nm})$, leading to significant effects on cell viability and cell activation (supporting text and Figure S2). In addition, a specific interaction of GO sheets with the cell membrane was noted whereby GO sheets adopted an arrangement parallel to the cell surface (designated as the cell "masking" effect). ${ }^{102}$ Another study based on two GO materials of different sizes ( 350 and $2000 \mathrm{~nm}$ ) showed opposite results. ${ }^{103}$ Both GO materials had no effect on cell viability and were internalized by cells in an energy-dependent process but showed different intracellular locations. In addition, except for IL-10, the release of all other cytokines-chemokines including IL-6, IL-12, TNF- $\alpha$, MCP-1, and IFN- $\gamma$ significantly increased after 2 days in cells exposed to large GO, whereas a weak increase was measured for small GO. ${ }^{103}$ The GO materials used in these two studies were obtained by the same Hummers' method, and the only difference that might explain the contrasting effects is the number of layers (single-layer GO in the former study and few-layer GO in the latter study). In another recent study, large GO showed a stronger adsorption onto the plasma membrane of macrophages when compared to small GO, and this elicited more robust interaction of Toll-like receptors (TLRs) and more potent activation of the NF- $\kappa \mathrm{B}$ pathway. ${ }^{104}$ Large GO was also shown to promote M1 polarization, associated with enhanced production of inflammatory cytokines and recruitment of immune cells. These size-dependent responses to GO were 
also evidenced in vivo. Hence, large GO was able to generate inflammatory responses significantly higher than those of small GO in mice after intraperitoneal injection. ${ }^{104}$ Naturally, careful characterization of the materials is crucial. In particular, endotoxin content must be controlled in any studies using immunecompetent cells. Recent work in the Graphene Flagship has focused on establishing a protocol for sterile production of GO according to Hummers' method. ${ }^{26}$ Using this protocol, endotoxin-free GO of differing lateral dimensions (50-300 nm and $10-40 \mu \mathrm{m}$, respectively, thickness $1-2 \mathrm{~nm}$ ) was produced, and cytotoxicity assessment as well as cytokine profiling was performed using primary human macrophages. ${ }^{39}$ These studies showed that small and large GO sheets were readily internalized by macrophages without any toxicity (Figure 3 ). Furthermore, GO did not trigger the production of pro-inflammatory TNF- $\alpha$ in this model. ${ }^{39}$ However, GO was found to elicit caspasedependent IL-1 $\beta$ expression, a hallmark of inflammasome activation, in LPS-primed macrophages. Moreover, a specific role of the inflammasome sensor, NLRP3, in GO-induced IL- $1 \beta$ secretion was demonstrated. In contrast to the above-mentioned study, ${ }^{104}$ the effects were independent of the lateral dimensions of GO. These differences could be ascribed to differences between the cell models, i.e., macrophage-like cell lines versus primary cells, as primary macrophages are far more efficient in terms of phagocytosis. It thus appears that inflammasome activation leading to IL- $1 \beta$ secretion may transpire for a range of carbon-based nanomaterials including carbon nanotubes as well as spherical particles and flat materials such as $\mathrm{GO}^{105-109}$ and other exogenous materials, ${ }^{100}$ indicating that the inflammasome functions as a universal "sensor" for xenobiotic agents. It is of interest to note that the commonly used adjuvant alum also triggers NLRP3-dependent release of IL- $1 \beta$ in macrophages. ${ }^{110}$ Thus, in analogy, the immunomodulatory effects of GBMs could perhaps be harnessed for biomedical uses. ${ }^{11}$

In a recent study, $\mathrm{Li}$ et al. examined a panel of GO materials prepared by a modified Hummers' method comprising pristine, rGO, and hydrated GO (hGO) in which quantitative assessment of the hydroxyl, carboxyl, epoxy, and carbon radical contents was used to study the impact on epithelial cells and macrophages as well as in the murine lung. ${ }^{112}$ The authors could show that hGO, which exhibited the highest carbon radical density, triggered cell death in THP-1 and BEAS-2B cells with attendant lipid peroxidation of the cell membrane, albeit at relatively high concentrations (up to $200 \mu \mathrm{g} / \mathrm{mL}$ ). The authors also demonstrated that hGO was more prone than the other materials to trigger lung inflammation, accompanied by lipid peroxidation in alveolar macrophages. ${ }^{112}$ Thus, carbon radical content plays an important role for toxicity of GO.

In addition to primary macrophages, the effects of GO were studied on macrophage-like cell lines. Chen et al. found that small GO $(350 \mathrm{~nm})$ induced the formation of small vacuoles in RAW264.7 cells without causing apparent cell death. ${ }^{13}$ Increasing the GO concentration triggered the formation of more vacuoles and significant cell death. In addition, GO treatment provoked TLR signaling and triggered consequent cytokine responses. Molecular analysis identified that TLR4 and TLR9 and their downstream signaling mediators MyD88, TRAF6, and NFkB played critical roles in the GO-induced inflammatory responses. ${ }^{113}$ This was confirmed in a subsequent study, in which necrotic cell death was shown to be mediated by activation of TLR4. ${ }^{114}$ In contrast, large GO (average lateral dimension $\sim 1 \mu \mathrm{m}$ ) did not activate TLR2 or TLR4 reporter cell lines, whereas single-walled carbon nanotubes (with or without a protein corona) activated TLR signaling with subsequent chemokine release. ${ }^{115}$ Protein adsorption biocorona formation is reminiscent of the process of opsonization whereby microorganisms or apoptotic cells are "tagged" for phagocytosis with antibodies, complement factors, or other soluble proteins. ${ }^{116,117}$ The impact of protein adsorption or biocorona formation on cell interactions of GBMs has been explored in a few studies using human cell lines. Hu et al. prepared GO using Hummers' method and measured the amount of the model protein, bovine serum albumin (BSA), adsorbed to GO. They found that the loading capacity was, respectively, $\sim 9$-fold and $\sim 1$-8-fold higher than that of BSA to multiwalled and single-walled carbon nanotubes. ${ }^{118}$ The data suggested that GO possessed an exceptionally high adsorption capacity arising from the $2 \mathrm{D}$ structure that provides a very high surface-to-volume ratio. In addition, GO possesses many surface defects that could serve as binding sites for proteins, and this could contribute to the observed differences in protein adsorption ability of GO and carbon nanotubes. In another study, protein adsorption was confirmed using experimental and theoretical approaches, and the authors proposed that protein-coated GO sheets lack the capacity for destructive membrane interactions due to the increase in the thickness of the GO sheets and reduction of the available surface area of GO, instead exposing largely hydrophilic surfaces that may lead to more benign interactions with membrane phospholipids. ${ }^{119}$ To improve the biocompatibility of pristine GO, $\mathrm{Xu}$ et al. prepared a series of GO derivatives including aminated GO (GO-NH$\left.H_{2}\right)$, poly(acrylamide)-functionalized $\mathrm{GO}$ (GOPAM), poly(acrylic acid)-functionalized GO (GO-PAA), and poly(ethylene glycol)-functionalized GO (GO-PEG) and compared their toxicity with that of pristine GO. ${ }^{120}$ Among these GO derivatives, GO-PEG and GO-PAA induced less toxicity than pristine GO, and GO-PAA was the most biocompatible material. The differences in biocompatibility were suggested to be due to the differential compositions of the protein corona, formed on their surfaces that determine their cell interactions and pro-inflammatory effects. ${ }^{120}$ In another recent study, coating of $\mathrm{GO}$ with complement factor $\mathrm{H}$ afforded almost complete protection ( $>90 \%$ reduction) against complement activation, suggesting that a "stealth" effect can be achieved through purposeful biocorona formation. ${ }^{121}$ By contrast, coating of GO with serum albumins achieved moderate protection ( $\sim 40 \%$ reduction), whereas immunoglobulin G amplified complement activation by several-fold.

PEGylation was shown in several studies to reduce the cytotoxic effects of GO on macrophages. ${ }^{122-124}$ However, a recent report suggested that PEGylation of small GO flakes (single-layer, $\sim 200 \mathrm{~nm}$ in lateral size) resulted in the stimulation of a potent cytokine response, despite not being internalized by macrophages. ${ }^{125}$ The authors performed extensive molecular dynamics simulations of pristine and PEGylated GO in the presence of lipid membranes. PEGylated GO appeared to preferentially adsorb onto and partially insert into cell membranes, thereby amplifying the interactions with stimulatory surface receptors. The authors also put forward the hypothesis that the integrin $\alpha_{\mathrm{v}} \beta_{8}$ is involved in initiating signal transduction related to the membrane binding of PEGylated GO. ${ }^{125}$ Overall, these results are surprising as they suggest that PEGylation does not lead to passivation but, instead, might lead to macrophage activation. Clearly, not only the characteristics of the parent material (GO) but also the surface modification (i.e., the morphology and density of PEG chains on the surface of GO) should 

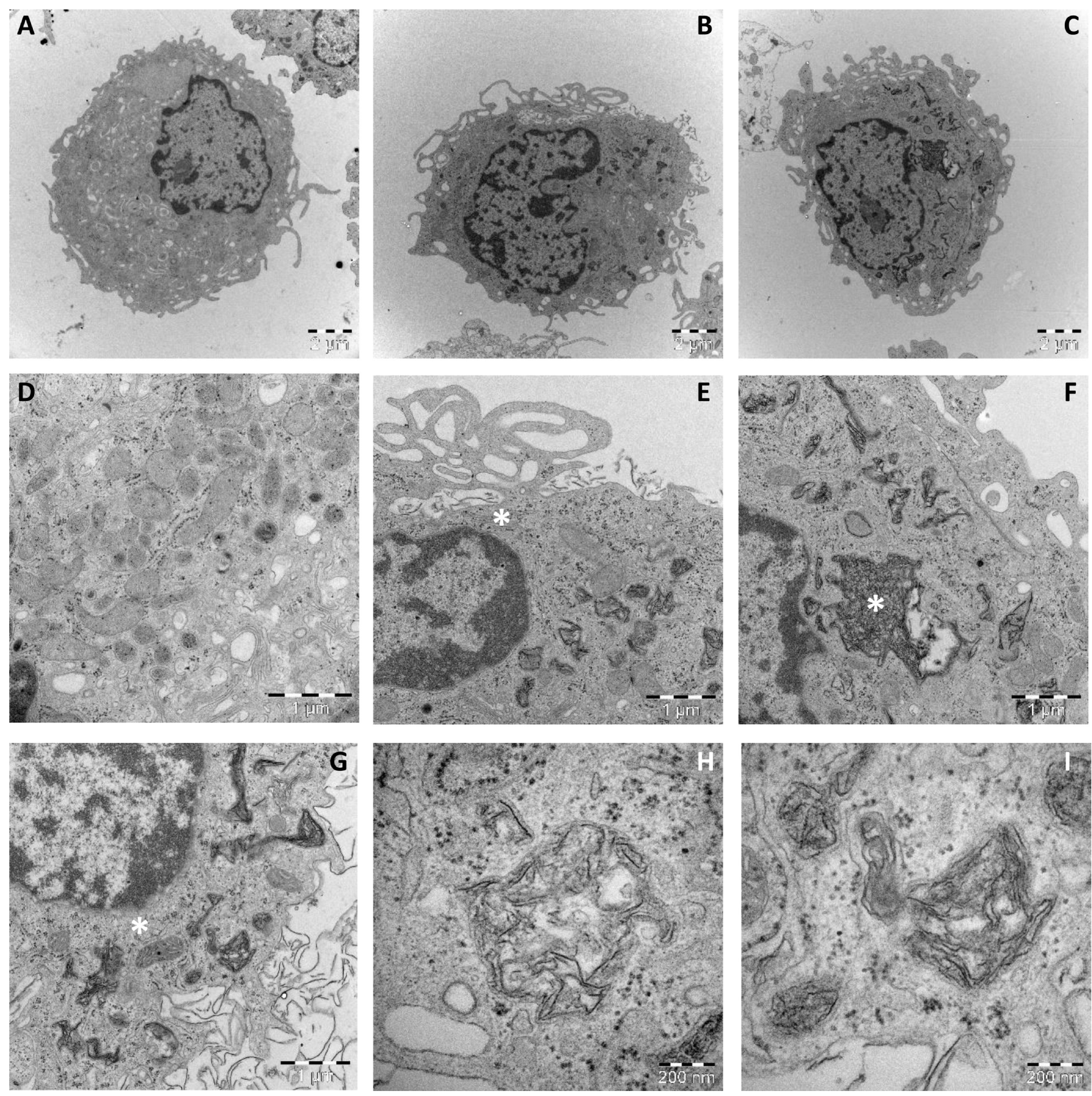

Figure 3. Macrophage uptake of GO. Primary human monocyte-derived macrophages readily ingest GO without ultrastructural signs of acute toxicity. Macrophages were incubated for $3 \mathrm{~h}$ with or without small or large GO $(50 \mu \mathrm{g} / \mathrm{mL})$. TEM images (scale bar: $2 \mu \mathrm{m})$ show (a) control cells, (b) cells exposed to GO-S, and (c) cells exposed to GO-L. Internalized GO can be seen in panels (b,c). Higher-magnification micrographs (scale bar: $1 \mu \mathrm{m}$ ) show (d) control cells, (e) cells exposed to GO-S, and (f,g) cells exposed to GO-L. The asterisk in panel (e) indicates GO sheets that are undergoing internalization. The asterisk in panel (f) shows a large aggregation of GO inside the cell, whereas the image in panel (g) shows the presence of GO sheets at the plasma membrane of the cell as well as GO internalized within the cell. The asterisk marks a mitochondrion, for comparison. Finally, at higher magnification (scale bar: $200 \mathrm{~nm}$ ), the micrographs in panels (h,i) show internalized GO-S and GO-L, respectively. Reprinted with permission from ref 39. Copyright 2018 Wiley-VCH Verlag GmbH \& Co.

be carefully evaluated, and endotoxin contamination should be excluded.

Macrophages belong to the front line of the innate immune defense against pathogens or foreign materials. ${ }^{111}$ Most studies on macrophages have been performed using macrophage-like cell lines or monocyte-derived macrophages. However, alveolar macrophages are likely one of the first cell types, along with epithelial cells, to interact with GBMs, reaching the lungs after pulmonary exposure. Studies on alveolar macrophages are scarce, but Weimann et al. performed studies using the rat NR8383 alveolar macrophage cell line as a model to predict the pulmonary toxicity of 18 different inorganic nanomaterials including graphite nanoplatelets and distinguish active from passive nanomaterials. ${ }^{126}$ Graphite nanoplatelets ( $<30 \mu \mathrm{m}$ flakes $)$ were classified as in vitro passive materials. Neutrophils are among the first cells to be recruited in the airways upon pulmonary exposure to GBMs 
and also play a key role in inflammation in many other tissues. Interestingly, a recent study has shown that when GO sheets interact with isolated human neutrophils, this triggers a dosedependent loss of cell viability and size-dependent formation of neutrophil extracellular traps (NETs). ${ }^{127}$ NETs consist of nuclear chromatin decorated with granule proteins such as neutrophil elastase (NE) and myeloperoxidase (MPO), and these structures are normally deployed by neutrophils for extracellular destruction of pathogens. In the latter study, the effects of GO were attributed to cholesterol oxidation in the plasma membrane, as evidenced by time-of-flight secondary ion mass spectrometry (ToF-SIMS) of exposed cells. ${ }^{127}$ The latter study underscores the importance of direct membrane interactions of 2D materials and implies that immune cells may respond to such materials in a manner that is comparable with immune responses to bacteria and fungi.

In contrast to GO, there are relatively fewer studies on graphene and its effects on the immune system. As graphene is too hydrophobic to obtain homogeneous dispersions in aqueous solutions, it is necessary to use appropriate biocompatible surfactants or coating molecules. In a recent study, Graphene Flaghsip scientists discovered that FLG obtained by solvent-free ball-milling treatment of graphite in the presence of melamine, subsequently dispersed in cell culture medium, is able to specifically kill monocytes while preserving the viability of macrophages. ${ }^{128}$ The capacity of FLG to trigger monocyte cell death was exploited to selectively kill monocytoid cancer cells isolated from patients affected by myelomonocytic leukemia. One of the most biocompatible surfactants used to disperse nanomaterials is pluronic F108. It has been found that exposure of macrophages to graphene in $1 \%$ pluronic decreases cell viability in a dose-dependent manner. This graphene significantly stimulated the secretion of Th1/Th2 cytokines and chemokines, and the morphology of näve macrophages was altered, with reduced capacity to adhere to the extracellular matrix and attenuated phagocytic capacity. ${ }^{129}$ The same type of material, again dispersed in $1 \%$ pluronic, can induce cytotoxic effects with dissipation of the mitochondrial membrane potential and increase of intracellular reactive oxygen species (ROS), resulting in apoptosis. ${ }^{130}$ Graphene or graphene that had undergone a direct oxidative process to introduce oxygenated species on its surface was also tested upon dispersion in physiological medium. Both materials do not cause any premature immune cell activation or suppression up to $75 \mu \mathrm{g} / \mathrm{mL}$ after $72 \mathrm{~h}$ of incubation. Macrophages showed relatively high intracellular uptake of oxidized, hydrophilic graphene compared to the hydrophobic graphene, which was found to be mainly retained on the cell surface and induced ROS-mediated apoptosis above $50 \mu \mathrm{g} / \mathrm{mL}$. ${ }^{131}$ When graphite is only partially exfoliated, a material in the micrometer lateral size range composed of multilayers of stacked graphene sheets (incorrectly called nanographite) can be isolated. Further treatment with strong acid generates oxidized (micro)graphite. Both types of (micro)graphite were shown to trigger a weak cytotoxicity with dose-dependent pro-inflammatory cytokine release. ${ }^{132} \mathrm{Li}$ et al. investigated the interactions of graphene and FLG microsheets with macrophages and other cell types and with model lipid membranes by combining molecular dynamics simulations with confocal fluorescence imaging and electron microscopic imaging. ${ }^{133}$ The imaging experiments suggested edge-first uptake of FLG into cells. The authors speculated that the ability of large graphene microsheets to penetrate and enter cells, documented experimentally and through simulations, may lead to cytoskeletal disruption, impaired cell motility, compromised epithelial barrier function, or other "geometric and steric effects". ${ }^{133}$ However, the lack of cell viability tests precluded any quantification of possible cellular damage.

High-throughput technologies have revolutionized the analysis of immune cells and their complex interactions. Consequently, a comprehensive analysis of how the immune system interacts with nanomaterials is only possible by the adoption of system biology approaches and high-throughput tools that permit multiplex analysis of cell type, cell activation state, and soluble mediators of stimulation/inhibition of immune cells. In a recent study conducted in the framework of the JTC 2015 FLAGERA call (G-Immunomics project), Orecchioni et al. investigated the effects on human immune cells of two types of thoroughly characterized GO sheets, differing in their lateral size distribution [small GO (GO-S) $100-500 \mathrm{~nm}$, and large GO (GO-L) $1-10 \mu \mathrm{m}$ ], by using a wide range of assays including whole-genome microarray analysis and single-cell mass cytometry. ${ }^{134}$ Exposure of peripheral blood mononuclear cells from healthy donors to small GO sheets was found to have a more significant impact when compared to large GO sheets, as reflected in the upregulation of critical genes implicated in immune responses and the release of the pro-inflammatory cytokines, IL- $1 \beta$ and TNF- $\alpha$. These findings were confirmed by genomics approaches using Jurkat $\mathrm{T}$ cells as representative for the adaptive immune system and THP-1 cells, a monocytic cell line representative of the innate immune system. The microarray studies identified the activation of some relevant immune pathways correlated with $\mathrm{T}$ cell chemotaxis/ $\mathrm{T}$ cell migration, regulation of $\mathrm{T}$ cell chemotaxis, and leukocyte chemotaxis pathways. ${ }^{134}$ This work suggested that small GO could elicit an innate and also an adaptive response boosting a strong recruitment of immune cells, potentially providing a first step toward unconventional strategies for nanobased immunotherapeutics. Moreover, by using single-cell mass cytometry, multidimensional cytometry experiments in which simultaneous investigations of 15 immune cell populations with interrogation of 30 markers at the single-cell level was possible. ${ }^{134}$ GO caused a broad, non-cell-specific activation triggering the production of all cytokines analyzed in a wide variety of cell populations. Following surface functionalization of the two GO with amino groups $\left(\mathrm{GO}-\mathrm{NH}_{2}\right)$, these materials resulted more specific, affecting the production of only a few cytokines in selected cell subpopulations. Taken together, these studies confirmed that the functionalization of GO significantly affected the number of transcripts altered by graphene. ${ }^{134}$ Moreover, functionalization of GO with amino groups increased the biocompatibility. The study lays the foundation for an innovative approach for multidimensional, high-throughput analysis of the effects of GBMs on immune cells (Figure 4).

Overall, when comparing the results of different studies on GBMs (above, and refer to Supporting Information), the toxicity of this class of materials toward macrophages, in particular, appears to be less pronounced as compared to the effects of carbon nanotubes. For other immune-competent cells, there are only a few studies available. However, studies performed in the Graphene Flagship have revealed differences in toxicity depending on the lateral dimensions of GBMs, though this hardly qualifies as a "fiber-like" effect. Furthermore, as we shall discuss in the following section, GBMs are susceptible to biodegradation, meaning that these materials are not biopersistant.

Biodegradation of Graphene-Based Materials. Biodegradation of nanomaterials is a topic of considerable relevance both in toxicology and in nanomedicine. The very fact that some carbon-based nanomaterials such as single-walled carbon 
a

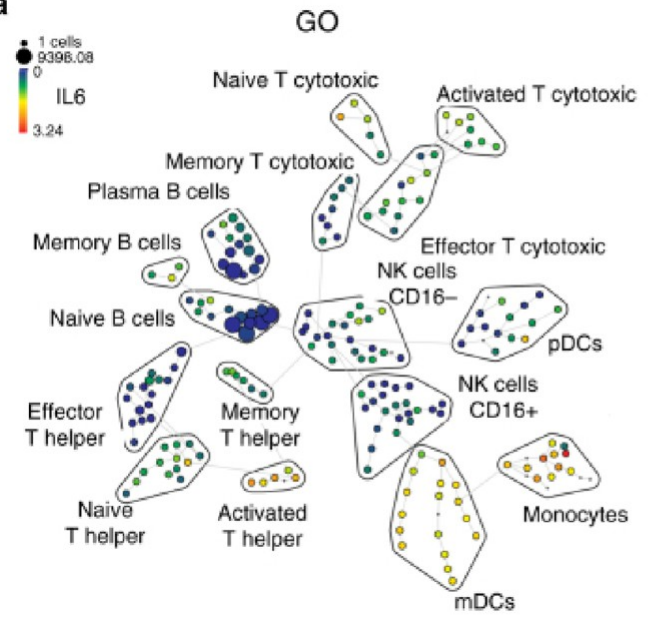

b

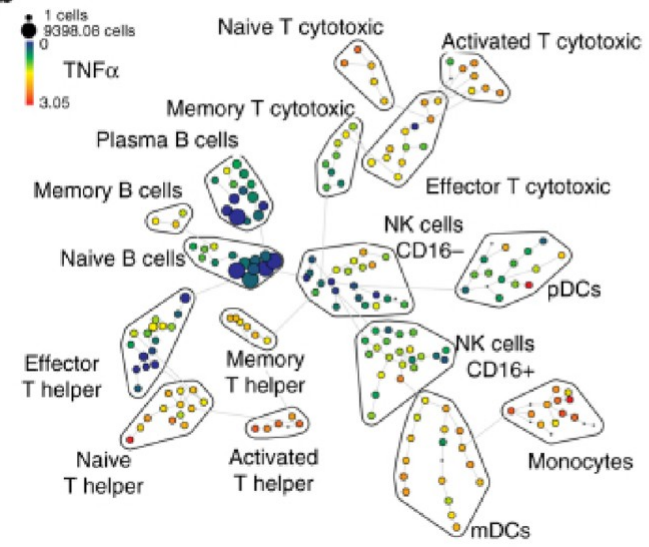

c

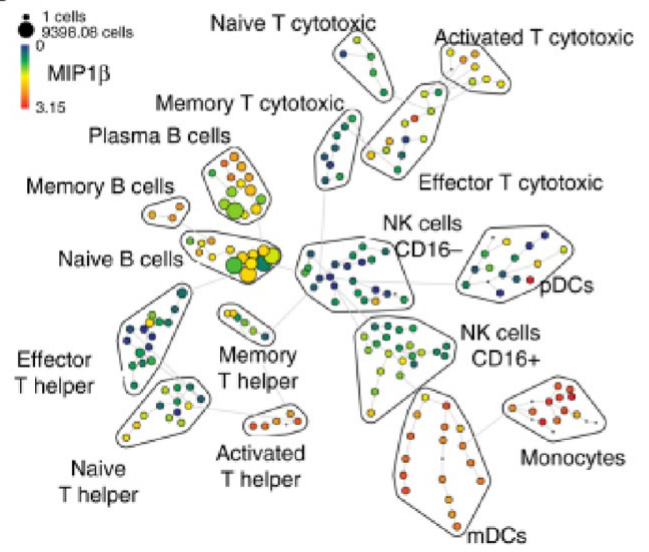

$\mathrm{GONH}_{2}$
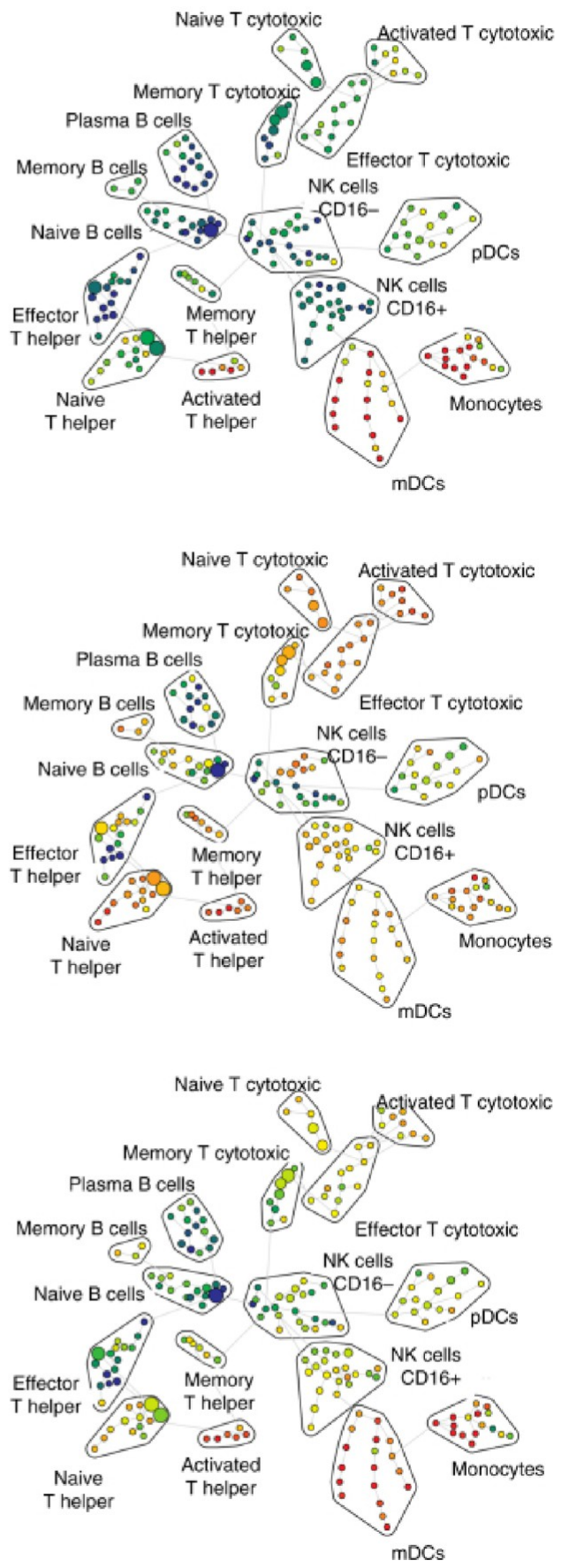

Figure 4. Dissecting the immunological impact of graphene using single-cell mass cytometry. SPADE (spanning tree progression analysis of density-normalized events) clustering algorithm analysis of significantly secreted cytokines. The tree plots show the different immune cell subpopulations, and the size of each cluster in the tree indicates the relative frequency of cells that fall within the dimensional confines of the node boundaries. Node color is scaled to the median intensity of marker expression of the cells within each node, expressed as a percentage of the maximum value in the data set: (a) IL-6; (b) TNF- $\alpha$, and (c) MIP-1 $\beta$ for GO (left) and GO-NH 2 (right). Reprinted with permission from ref 134. Copyright 2017 Nature Publishing Group.

nanotubes are susceptible to degradation by immune cells sets them aside from asbestos, which is nondegradable in biological systems. ${ }^{9}$ Although some research has been done on GBMs in this regard, the description of the structural characteristics of the different materials used in each biodegradation study is crucial to rationalize the results. Here, we focus our discussion on the degradation of GBMs, emphasizing the role of the three fundamental material properties, i.e., the number of graphene layers, the average lateral dimension, and the atomic $\mathrm{C} / \mathrm{O}$ ratio (Figure 1) ${ }^{22}$ (supporting text and Figure S3).

Kotchey et al. found that low concentrations of horseradish peroxidase (HRP) could degrade GO, whereas reduced GO was not affected. ${ }^{135}$ The different interactions between GO or rGO with the active site of HRP was proposed as the main reason for these differences in susceptibility to degradation. Zhang et al. studied the effect of graphene, GO, and rGO on the stability of 
HRP. Interestingly, whereas both graphene and GO were found to reduce enzyme stability, rGO was able to preserve the catalytic activity of the enzyme, presumably by scavenging superoxide radicals, thereby protecting the enzyme from oxidation. ${ }^{136} \mathrm{Li}$ et al. examined how surface coatings affect the cytotoxicity and biodegradation of GO and its derivatives. ${ }^{137}$ Although pristine GO triggered significant toxicity to macrophage-like U937 cells (albeit at very high doses, i.e., 100 or $200 \mu \mathrm{g} / \mathrm{mL}$ ), coating of GO with PEG or BSA attenuated the toxicity. On the other hand, both PEG- and BSA-coated GO were found to be resistant to HRP-induced biodegradation. ${ }^{137}$ To circumvent this, the authors conjugated PEG to GO via a cleavable disulfide bond, obtaining GO-SS-PEG with negligible toxicity and considerable degradability when incubated with HRP and $\mathrm{H}_{2} \mathrm{O}_{2}$. Functionalization can be also exploited to design systems able to "attract" enzymes toward the modified GO surface. In a recent study from the Graphene Flagship, Kurapati et al. demonstrated enhanced degradation of GO when functionalized with coumarin and catechol, which are natural ligands of HRP. ${ }^{138}$ Kurapati et al. also demonstrated that MPO, a human enzyme secreted by activated neutrophils, can degrade GO. In this case, aqueous dispersibility of the material was found to play a crucial role in the biodegradation process. ${ }^{139}$ Three different GOs with similar lateral dimensions and $\mathrm{C} / \mathrm{O}$ ratios but different thicknesses were evaluated, and the two highly dispersed materials were completely or almost completely degraded at $24 \mathrm{~h}$, whereas only limited structural changes occurred in the case of the aggregated sample. ${ }^{139}$ More recently, Graphene Flagship scientists have reported that single-layer GO sheets of differing lateral dimensions could be readily degraded by isolated human neutrophils stimulated to produce NETs or activated to undergo degranulation with the release of MPO. ${ }^{140}$ In addition, the degradation byproducts of GO were shown to be nongenotoxic to human lung cells. Based on these in vitro results, it is possible to suggest that inhalation of GO with the recruitment of neutrophils and macrophages may potentially lead to intra- or extracellular digestion of GO, which could mitigate the overall pulmonary impact, as previously shown for certain carbon nanotubes. ${ }^{9}$ Indeed, it was demonstrated that oxidation and clearance of single-walled carbon nanotubes from the lungs of MPO-deficient animals after pharyngeal aspiration was markedly less effective after 28 days, whereas the inflammatory response was more pronounced than in wild-type $\mathrm{C} 57 \mathrm{Bl} / 6$ mice. $^{141}$ Further studies to assess the in vivo degradation and clearance of GO are warranted.

Girish et al. assessed the degradation of graphene in vivo. ${ }^{91}$ Prior to administration, the carboxyl-functionalized graphene of lateral size $\sim 200 \mathrm{~nm}$ was shown to be well-dispersed in aqueous medium, but $24 \mathrm{~h}$ postinjection, large aggregates of sizes up to $10 \mu \mathrm{m}$ could be detected in various organs. Confocal Raman imaging was conducted to identify the gradual development of structural disorder occurring over a period of 3 months in lung, liver, kidney, and spleen of mice exposed by i.v. injection to graphene. The authors argued that graphene degradation was mainly orchestrated by macrophages in the different organs. ${ }^{91}$ The specific mechanism involved was not disclosed, but it is relevant to note that previous studies have shown that singlewalled and multiwalled carbon nanotubes can be digested by macrophages through a peroxynitrite-driven pathway that depends on the activation of NADPH oxidase and iNOS. ${ }^{142,143}$

The degradation potential of GO has been also studied using acellular oxidative systems such as the photo-Fenton reaction ${ }^{144}$ or sodium hypochlorite $(\mathrm{NaClO})$, colloquially known as bleach. $^{145}$ In the former case (oxidation potential of $\mathrm{OH}^{\bullet}=$ $2.80 \mathrm{~V}$ ), only 3 days were sufficient to completely degrade GO. ${ }^{144}$ Using FTIR, mass spectrometry, and NMR, potential structures for the oxidation products, which consisted of oxidized polycyclic aromatic hydrocarbons, were proposed. Seven days were instead required to degrade $\mathrm{GO}$ using $1 \% \mathrm{NaClO}$ in water (oxidation potential $\mathrm{HClO} / \mathrm{Cl}^{-}=1.48 \mathrm{~V}$ ). ${ }^{145}$ However, GO was degraded more rapidly than oxidized multiwalled carbon nanotubes. Microbial degradation is another promising way to degrade GBMs as this does not require controlled conditions such as controlled temperature and $\mathrm{pH}$. Lalwani et al. reported that oxidized and reduced GO nanoribbons (GO-NR and rGO-NR) were degraded by lignin peroxidase, an enzyme released from white rot fungus. ${ }^{146}$ Raman spectroscopic analysis indicated that, within 96 h, GO-NR and rGO-NR were completely and partially degraded by lignin peroxidase, respectively. Furthermore, Liu et al. demonstrated that naphthalene-degrading bacteria could degrade graphitic materials due to a mechanism based on electron transfer. ${ }^{147}$ Interestingly, graphite, GO, and rGO showed different rates of oxidation and degradation after being incubated with the bacterial strain for 14 days. Taken together, these studies suggest the potentiality of degrading GBMs released in the environment.

In conclusion, studies conducted in the past several years have clearly shown that intra- and extracellular degradation of GO can be executed by immune cells. However, more research is required on the degradation of other GBMs with defined properties using relevant in vitro and in vivo models to better understand the possible risks of long-term biopersistence of such materials and in order to make them safer-by-design.

Dermal Effects of Graphene-Based Materials. The skin is the major barrier between the human body and the environment, and it can be considered as one of the most important exposure sites for GBMs, not least in the occupational setting. ${ }^{148,149}$ Given the chemical nature of GBMs, skin irritation may be considered as the most feasible outcome after cutaneous exposure. However, skin sensitization cannot be excluded in light of the propensity of GBMs to interact with proteins. ${ }^{150,151}$ Indeed, cutaneous contact with related materials such as graphite and carbon nanotubes has been associated with skin disorders, e.g., contact dermatitis. ${ }^{152,153}$

Limited toxicological data are currently available for GBMs at the skin level. The majority of studies are in vitro studies on skin keratinocytes and/or fibroblasts. Liao et al. evaluated the impact of a panel of different GOs obtained by Hummers' method (lateral dimension: $871-1678 \mathrm{~nm}$; thickness: $1-10 \mathrm{~nm}$ ) and graphene sheets produced by dehydration process of GO (average lateral dimension: $4312 \mathrm{~nm}$; thickness: up to $10 \mathrm{~nm}$ ) on skin fibroblasts. ${ }^{66}$ The authors demonstrated that, after $24 \mathrm{~h}$ exposure, graphene induces a cytotoxic effect higher than that of $\mathrm{GO}$, probably due to a more pronounced tendency to aggregate. In subsequent studies, carried out in the framework of the Graphene Flagship, the effect of three GOs obtained by Hummers' method (lateral dimensions: 622-979 nm) and one FLG sample (average lateral dimension: $552 \mathrm{~nm}$ ) in spontaneously immortalized human keratinocytes was evaluated. ${ }^{154}$ All materials induced a significant cytotoxic effect, albeit with different potencies. The cytotoxic potential was mainly dependent on the oxidative state of the GBMs, with FLG being significantly less cytotoxic than GO. In addition, using various in vitro assays, the results demonstrated that FLG and GOs were able to induce a significant mitochondrial dysfunction after a sustained plasma membrane damage. ${ }^{154}$ These effects seem to be dependent 
on a significant production of reactive oxygen species through a selective activation of flavoprotein-based oxidative enzymes, such as NADH dehydrogenase and xanthine oxidase, by FLG (average lateral dimension: $391 \mathrm{~nm}$ ) and GO (average lateral dimension: $979 \mathrm{~nm}) .{ }^{155}$ These effects are likely due to a closer interaction with the plasma membrane, as suggested in a previous study using molecular dynamics simulations as well as electron microscopy imaging, showing the ability of microsize graphene to interact with the plasma membrane of primary human keratinocytes. ${ }^{133}$

Only one in vivo study has been published thus far on dermal effects of GBMs. In this study, GO (lateral dimension: 250-1750 nm; thickness: $2 \mathrm{~nm}$ ) was injected into the dermis of the growing feather sites of chickens, providing a minimally invasive model for the investigation of the local immune/ inflammatory reaction. ${ }^{156}$ The response was evaluated based on the type and relative amount of leukocytes at the site of intradermal injection. The results showed an increased infiltration of lymphocytes and macrophages at the injected site up to 2 days postinjection decreasing gradually between days 4 and 7 . Qualitative and quantitative aspects of the leukocyte infiltration suggested a cell-mediated immune response, probably initiated by GO interactions with host proteins. ${ }^{156}$ Even though the available data are not sufficient to define the relevant toxicity of GBMs after cutaneous exposure, their ability to initiate an immune response after dermal injection raises concern for GBM uses.

Overall, the currently available literature, limited to one in vivo and a few in vitro studies, is not sufficient to draw any conclusions on the hazard related to dermal exposure to GBMs.

Pulmonary Effects of Graphene-Based Materials. Among the different routes of unintended exposure to nanomaterials in occupational settings, inhalation is the route of highest concern. A long history of studies on the impact of air pollution and ultrafine particles on human pulmonary health has supported the notion that nano- and micron-sized particles may cause harm to the lungs (reviewed in ref 97). GBMs are not exempt from these concerns as these materials are commercially available as volatile powders often referred to as nanopowders or suspensions/dispersions of graphene nanoplatelets (GNPs), $\mathrm{GO}$, or rGO-most often water-based. This section will focus on studies investigating GBM pulmonary effects after exposure of the pulmonary system via the common routes (i.e., intratracheal, oropharyngeal, inhalation) or using models reproducing potential pulmonary exposure consequences (i.e., intrapleural, intraperitoneal). Pulmonary effects of GBMs injected intravenously will not be discussed here, as this topic has been reviewed previously by others. ${ }^{18,157}$

Roberts et al. evaluated the pulmonary and systemic toxicities of three types of graphite plate-like structures $(20 \mu \mathrm{m} / 72$ layers, $5 \mu \mathrm{m} / 84$ layers, $1-2 \mu \mathrm{m} / 28$ layers) in mice following pharyngeal aspiration. ${ }^{158}$ Toxicity was dose-dependent, with the lowest dose $(4 \mu \mathrm{g} /$ mice $)$ inducing no toxic response, whereas the highest dose $(40 \mu \mathrm{g} /$ mice $)$ caused size-dependent lung inflammation. The adverse pulmonary and systemic effects observed at early time points at the highest dose showed signs of resolution for all materials. Despite the persistence of all three materials, neither fibrosis nor granulomatous lesions were observed. The authors highlighted that not only size but also surface reactivity and agglomeration are important to consider when studying GBMs. ${ }^{158}$ Schinwald et al. studied large graphene platelets (1-10 $\mu \mathrm{m}, 10$ layers) following oropharyngeal instillation in mice to assess their pulmonary effects. ${ }^{159}$ At 1 day, large numbers of polymorphonuclear leucocytes, mainly neutrophils and eosinophils, were recruited into the lungs, and cytokine levels were increased. Due to the shape of graphene platelets, the authors hypothesized that their uptake by macrophages could be impaired, leading to "frustrated phagocytosis". Indeed, examination of pleural macrophages of exposed mice showed signs of frustrated phagocytosis after exposure to graphene platelets, whereas nanoparticulate carbon black was fully taken up by cells. ${ }^{159}$ However, using the same materials, the same authors did not observe inflammation at 7 days or 6 weeks postexposure following pharyngeal aspiration. ${ }^{160}$ At these time points, there were no longer any signs of inflammation in the lungs. Similarly, there was no fibrosis despite the obvious persistence of large amounts of graphene platelets in the airways. Importantly, there was no sign of the materials in the pleural cavity (neither after 1 day nor after 6 weeks). This result thus indicated that graphene platelets were not able to translocate into the pleural space, thus preventing them from inducing granuloma on the pleural mesothelium, a hallmark of asbestos pathogenicity. On the other hand, when injected directly into the intrapleural space, graphene platelets induced the formation of large granuloma (indicative of inflammation) at the surface of the pleural mesothelium and non-adhesive rosette-like cell/particle agglomerates (indicative of frustrated phagocytosis) in the pleural cavity. ${ }^{159}$

Mao et al. administrated FLG (lateral dimension: 60-590 nm; thickness: $1-4 \mathrm{~nm}, \mathrm{C} / \mathrm{O}$ ratio: 14.8$)$ to mice via intratracheal aspiration $^{83}$ (supporting Figure S4). Whereas the low dose $(5 \mu \mathrm{g} /$ animal $)$ did not cause any injury to the lungs, the high dose (50 $\mu \mathrm{g} / \mathrm{animal})$ induced cell recruitment and lung damage. Histopathological analysis of the lung sections after 1 day confirmed the absence of damage after exposure to low doses, despite the material burden. In contrast, lungs exposed to the high dose of FLG showed interstitial and parenchymal damages, with a large amount of macrophages in alveoli at day 1 . The lungs exposed to a high dose of FLG recovered slowly, with damage still present after 7 days but disappearing by day 28 , despite a persistent burden estimated at almost half of the initial dose. ${ }^{83}$ Similarly, persistence in the lungs up to 28 days after single bolus exposure was observed in another study of FLG nanoplatelets (average dimension: $325 \mathrm{~nm}$; thickness: $3-4 \mathrm{~nm}$ ). ${ }^{161}$ Despite the persistence of the materials, no lung lesions (e.g., granuloma or fibrosis) were observed. In a follow-up study, animals were exposed to similar FLG and assessed at day 90 after a single bolus exposure. ${ }^{162}$ There was an increase of the percentage of lymphocytes in BAL fluid of animals treated with the lowest doses and an increase in the total number of cells and apoptotic cells in the BAL fluid of animals treated with the highest dose. Elevated levels of cytokines and chemokines were also found 90 days after exposure in the high-dose-treated animals. The authors concluded that the pulmonary persistence of materials, in part within macrophages, was the reason for the pulmonary and systemic immune responses observed at the highest dose tested. $^{162}$

The pulmonary effects of GNPs were also evaluated in animals exposed via inhalation, which is the gold standard method for assessing pulmonary toxicity. In a first study, rats were exposed for $6 \mathrm{~h} /$ day for 5 days at 0.68 or $3.86 \mathrm{mg} / \mathrm{m}^{3}$ graphene (lateral size: $550 \mathrm{~nm}$, thickness: $8 \mu \mathrm{m}, \mathrm{C} / \mathrm{O}$ ratio: 9.8 ), resulting in deposited doses of 18 or $102 \mu \mathrm{g}$, respectively. ${ }^{163}$ Despite the observation of graphene-laden macrophages, no effects on BAL cell composition or $\mathrm{LDH}$ release (indicative of lung damage) were seen at $1,3,7$, or 28 days postexposure. In a second study by the same authors, rats were exposed to GNP (lateral sizes up to $2 \mu \mathrm{m}, 20-30$ layers, C/O ratio: 25 ) for $6 \mathrm{~h} /$ day, 5 days/week, for 4 weeks, at $0.12,0.47$, and $1.88 \mathrm{mg} / \mathrm{m}^{3}$, leading to an 
estimated deposited doses of 12,50 , and $198 \mu \mathrm{g}$, respectively. ${ }^{164}$ The animals were assessed at 1,28 , and 90 days after exposure. Inhaled materials were found in macrophages, but no signs of inflammation were noticed at any time points, regardless of the doses applied. Interestingly, inhaled materials were also found in the mediastinal lymph nodes, suggesting translocation of materials from the airways to the lymphatic system. ${ }^{164}$ Translocation of GBMs to mediastinal lymph nodes was also observed after exposing animals to various types of pristine or functionalized GNPs by intratracheal instillation. ${ }^{165}$ Moreover, an early inflammatory response with recruitment of neutrophils was observed, and this was more pronounced for the aminofunctionalized materials.

Oxidized graphene derivatives have also been investigated for their potential impact on the lungs. Nanoscale GO sheets (lateral dimension: $10-800 \mathrm{~nm}, 1-2$ layers) were intratracheally instilled in mice, and their impact on lungs was assessed at various time points from 1 day and up to 3 months. ${ }^{82}$ Inflammation appeared already at day 1 , with a dose-dependent recruitment of immune cells including neutrophils, along with signs of acute lung injury. The peak of the response was observed at 2 days, returning to levels close to, but still above, control levels by day 7. Material-laden macrophages started to appear in lung sections by day 7 and were still present in the lungs at 3 months, though a decrease in the blackness of the lungs from day 1 to day 90 suggested an ongoing clearance process. The pulmonary effects of GO sheets dispersed in water $(0.5-2 \mathrm{~nm} /$ $1 \mathrm{~nm}$ ) were compared to those of GNPs dispersed in water $(1.2-5 \mathrm{~nm} / 1-5 \mathrm{~nm})$ or in $2 \%$ pluronic F108 in water (1.2$5 \mathrm{~nm} / 1-5 \mathrm{~nm}){ }^{68}$ GO-treated animals exhibited severe pulmonary inflammation but no signs of fibrosis. In contrast, GNPs were less inflammogenic, and this was further minimized when the GNPs were well-dispersed using the block copolymer pluronic. The authors suggested that oxidation of graphene is a major contributor to its pulmonary toxicity. ${ }^{68}$ This is in contrast to a previous study in which colloidally stable dispersions of single-layer GO $(<500 \mathrm{~nm})$ were injected i.p. into mice without signs of inflammation or granuloma formation onto the peritoneal mesothelium. ${ }^{35}$ Single intratracheal administration of either GO sheets (lateral dimension: $2-3 \mu \mathrm{m}$, thickness: $1-2 \mathrm{~nm}, \mathrm{C} / \mathrm{O}$ ratio: 1.4 ) or rGO sheets (lateral dimension: $1-2 \mu \mathrm{m}$, thickness: $1-2 \mathrm{~nm}, \mathrm{C} / \mathrm{O}$ ratio: 8.5 ) was performed in mice. ${ }^{166} \mathrm{GO}$ sheets induced a strong neutrophil influx at 18,54 , and $162 \mu \mathrm{g} /$ mouse, 1 and 3 days postexposure. This was paralleled by a pulmonary acute phase response. In contrast, rGO sheets induced significantly less neutrophil influx, and neutrophil influx was only statistically significantly increased at $162 \mu \mathrm{g} /$ mouse. However, all three dose levels induced statistically significantly increased neutrophil influx at 90 days postexposure. Carbon black nanoparticles "Printex 90" which have a specific BET surface area similar to the studied rGO sheets were included as benchmark particles. The inflammatory response to rGO sheets was lower than or similar to the response to carbon black, whereas on day 1 and 3, the inflammatory response to GO sheets was stronger than the inflammatory response to carbon black. Additionally, DNA damage in BAL fluid cells was found for both GO and rGO sheets, whereas no genotoxicity was observed in lung or liver tissues. ${ }^{166}$ This is interesting as it contrasts with previous results obtained in vitro for the same GO and $\mathrm{rGO}$ materials, using the murine lung epithelial cell line FE1. ${ }^{167}$ Obviously, lung epithelial FE1 cells are very different from BAL cells (i.e., a mixture of immune-competent cells), and this simple difference could explain the discrepancy between the two studies.
However, similar genotoxic effects have been reported for some multiwalled carbon nanotubes. ${ }^{168,169}$ On the other hand, degradation of GBMs has been demonstrated in vitro and in vivo, ${ }^{10}$ and a recent study showed no genotoxicity in lung cells exposed to the degradation products following MPO-mediated digestion of GO. ${ }^{140}$

GBMs are produced with a range of different lateral dimensions, and it is important to investigate the role of size on pulmonary effects, not least because other carbon-based materials including carbon nanotubes clearly display a size-dependent toxicity. ${ }^{\circ}$ Ma et al. produced single-layer GO sheets of three distinct lateral dimensions (50-400, 300-800, and 700-1400 nm), though only the larger and smaller ones were tested for their pulmonary effects in mice. ${ }^{104}$ Nevertheless, a clear size-dependent response was observed 3 days after exposure for all parameters tested (i.e., LDH and protein content, cytokine levels, cell recruitment in BAL fluid), with the larger materials causing more adverse effects than the smaller ones. This is in apparent contrast to a recent in vitro study using primary human macrophages, in which neither small $(50-300 \mathrm{~nm})$ or large $(10-40 \mu \mathrm{m}) \mathrm{GO}$ sheets were found to trigger inflammatory cytokines, unless the cells were primed first with LPS. ${ }^{39}$ Unfortunately, the authors of the aforementioned in vivo study performed the investigations only after 3 days and did not report on the histopathological analysis, leaving open the question of long-term consequences of the acute inflammatory response, including potential induction of fibrosis. In contrast, Wang et al. compared large $(1676 \mathrm{~nm})$ versus small $(179 \mathrm{~nm})$ GO sheets and BSA-dispersed $(640 \mathrm{~nm})$ versus pluronic F108 dispersed (45 nm) GNPs and reported that all materials, with the exception of the GNPs dispersed in pluronic F108, induced collagen deposition/fibrosis 21 days after pharyngeal aspiration. ${ }^{106}$ Overall, F108-dispersed GNPs were less inflammogenic and not fibrogenic compared to BSAdispersed GNPs, which were both inflammogenic and fibrogenic, whereas both small and large GO sheets were inflammogenic and fibrogenic, and large GO sheets induced more pronounced effects than the small GO sheets. Recent studies performed in the Graphene Flagship have revealed similar sizedependent responses in the lungs of mice exposed via the intranasal route to a single administration of small (lateral dimension: $170 \mathrm{~nm}$, thickness: $1 \mathrm{~nm}, \mathrm{C} / \mathrm{O}$ ratio: 2.2$)$ or large $(1723 \mathrm{~nm}, 1 \mathrm{~nm}$, $\mathrm{C} / \mathrm{O}$ ratio: 2.2 ) GO sheets. ${ }^{170}$ In this study, the large GO sheets induced more immune cell infiltration (primarily neutrophils) in the lungs at day 1 when compared to small GO sheets, leading to the formation of granuloma by day 7 , which increased by day 28 . However, in contrast to the previous study, ${ }^{106}$ no fibrosis was observed after 28 days despite the presence of granulomas in the lungs of animals treated with large GO. ${ }^{170}$ In another recent study, the degree of surface oxidation of GO was evaluated in relation to pulmonary toxicity. ${ }^{112}$ Hence, GO sheets (lateral dimension: $334.1 \mathrm{~nm}, 1$ layer, C/O ratio: 1.72 ) were compared to $\mathrm{rGO}$ sheets (lateral dimension: $549.2 \mathrm{~nm}, 3$ layers, C/O ratio: $5.06)$ or hydrated GO sheets $(329.8 \mathrm{~nm}, 3$ layers, C/O ratio: $2.60)$. The hydrated materials produced the highest amount of carbon radicals and induced the highest production of ROS, whereas the reduced materials induced the lowest amount of free radicals and ROS. The pulmonary impact was evaluated in mice $40 \mathrm{~h}$ after a single pharyngeal aspiration exposure at $2 \mathrm{mg} / \mathrm{kg}$ for each of the three materials. The GBMs with the highest pro-oxidative potential (hydrated GO) caused more lung damage in this acute model. ${ }^{112}$ Furthermore, GO and GNP were aerosolized onto the lung epithelial tissue surface in a $3 \mathrm{D}$ human lung model. ${ }^{171}$ Subsequent evaluation showed that exposure 
to GBMs at two different exposure concentrations ( $\sim 300$ and $\sim 1000 \mathrm{ng} / \mathrm{cm}^{2}$ ) did not elicit any adverse effects in the 3D lung model.

Finally, whereas in vivo studies are typically performed in healthy laboratory animals, susceptible models of disease should also be investigated. Shurin et al. employed a classical model of asthma in which ovalbumin (OVA) is used as allergen to study the impact of single-layer GO sheets (lateral dimension: $20 \mathrm{~nm}$ to $5 \mu \mathrm{m}) .{ }^{172} \mathrm{GO}$ instilled oropharyngeally $(80 \mu \mathrm{g} /$ mouse $)$ was found to modulate the allergic inflammation by decreasing the Th2-mediated immune response, leading to increased airway hyper-reactivity and remodeling. Interestingly, without coexposure to allergens, GO sheets did not induce any significant adverse effects. Exposure to GO during sensitization with OVA decreased eosinophil accumulation and increased the recruitment of macrophages in BAL fluid. In addition, GO increased alveolar macrophage production of the asthma-associated chitinases, CHI3L1 and AMCase. ${ }^{172}$

In conclusion, it appears that the extent of pulmonary impact is directly correlated to the specific physicochemical properties of the tested materials. Dimensions seem once again to be an essential driver of the biological response to GBMs. Of note, only few studies so far have reported the induction of fibrosis, a hallmark of lung damage, after pulmonary exposure to GBMs. The lack of pulmonary fibrosis is an important difference when comparing GBMs with pathogenic multiwalled carbon nanotubes (i.e., those classified by IARC as potential human carcinogens). ${ }^{6}$ However, further systematic investigations looking at long-term impact of GBMs are warranted to fully address this issue.

Cardiovascular Effects of Graphene-Based Materials. Over the past 20 years, there has been compelling evidence of a relationship between air pollution, inhalation of fine and ultrafine particles, their impact on the lungs, and cardiovascular diseases. ${ }^{173,174}$ Hence, inflammation and oxidative stress in the pulmonary system resulting from the inhalation of particulate matter are cited as probable causes for collateral effects on the cardiovascular system. Despite the established connection between pulmonary exposure and cardiovascular disease, information regarding the possible cardiovascular impact of inhalable nanomaterials remains limited. ${ }^{175}$ For GBMs, few studies so far have reported on cardiovascular impacts after pulmonary exposure. Following a single intratracheal instillation of GO (lateral dimension: $2-3 \mu \mathrm{m}, 2-3$ layers, $\mathrm{C} / \mathrm{O}$ ratio: 1.4$)$ compared to rGO (lateral dimension: $1-2 \mu \mathrm{m}, 2-3$ layers, $\mathrm{C} / \mathrm{O}$ ratio: 8.5 ), Bengtson et al. measured acute phase response proteins, biomarkers for risk of cardiovascular disease. ${ }^{166}$ Unlike rGO, GO sheets clearly induced a transient acute phase response, with significant increase of these biomarkers at day 1 and day 3, disappearing by day 28 or day 90 . In another study, increased expression of the acute phase gene encoding SAAl in the liver was found after pharyngeal aspiration of graphite platelets. ${ }^{158}$ These limited results stress the need for further investigations regarding the potential impact of GBMs on the cardiovascular system after inhalation. In addition to these studies, there are only a few studies on the interactions of GBMs with cells of the cardiovascular system. For instance, myocardial H9c2 cells were exposed to GO (lateral dimension: $380 \mathrm{~nm}, \mathrm{C} / \mathrm{O}$ ratio: 0.82 ) or rGO (lateral dimension: $150 \mathrm{~nm}, \mathrm{C} / \mathrm{O}$ ratio: 1.70 ) in a recent study. ${ }^{176}$ Cytotoxicity was dose-dependent above $10 \mu \mathrm{g} / \mathrm{mL}$, and $\mathrm{rGO}$ was found to be more toxic than GO and was internalized to a greater extent than GO. Singh et al. studied GO sheets (lateral dimension: $0.2-5 \mu \mathrm{m}$ ) and noted strong activation and aggregation of platelets with activation of Src kinases and release of calcium from intracellular stores. ${ }^{177}$ Furthermore, intravenous injection of GO $(250 \mu \mathrm{g} / \mathrm{kg})$ was found to induce extensive pulmonary thromboembolism in mice $15 \mathrm{~min}$ after administration of the material. For comparison, reduced GO was significantly less effective in aggregating platelets in the lung vasculature. The authors argued that variations in surface properties may be responsible for the observed differences between the two materials. ${ }^{177}$ However, they did not address the possible role of the biocorona formed on GO or rGO, though it is likely that both materials are "coronated" upon contact with blood. ${ }^{10}$ In contrast, GNPs functionalized with amine groups did not activate isolated human platelets and did not induce pulmonary thromboembolism in mice after i.v. administration. ${ }^{178}$ In addition, these amine-bearing GNPs did not cause hemolysis of isolated human RBCs for concentrations as high as $10 \mu \mathrm{g} / \mathrm{mL}$, whereas $\mathrm{GO}$ sheets caused $\mathrm{RBC}$ membrane rupture even at the lowest concentration $(2 \mu \mathrm{g} / \mathrm{mL}){ }^{178}$

On the other hand, in biodistribution studies conducted in the Graphene Flagship, no obvious acute cardiovascular or hematological adverse effects were noted upon i.v. administration of single- to few-layer GO sheets ${ }^{41,95}$ or multilayered GO in mice. ${ }^{40}$ $\mathrm{Qu}$ et al. reported that PBS-based and PBS-1\%Tween 80-based suspensions of GO did not cause thromboembolism in the lungs of mice following i.v injection. ${ }^{89}$ Furthermore, GNPs and acidoxidized GNPs induced neither hemolysis nor activation and aggregation of platelets. ${ }^{131}$ Following i.v. administration, PEGGO did not trigger hemotoxicity over a 3 month period. ${ }^{67}$ A recent study showed that whereas GO sheets caused hemolysis, coating the GO sheets with lipid-based vesicles mitigated this effect. ${ }^{179}$

Overall, the lack of consistency between published studies underlines the need to correlate biological effects with physicochemical properties and suggests that further studies on the potential impact of GBMs on the blood and cardiovascular system are needed.

Gastrointestinal Effects of Graphene-Based Materials. The gastrointestinal (GI) system enables organisms to take in food, digest it to extract and absorb nutrients and essential elements, as well as expel the remaining waste as feces. There are two major sources of potential oral exposure to nanomaterials: (i) direct ingestion materials present in food or released from food packaging and (ii) indirect ingestion of inhaled materials. ${ }^{180,181}$ This means that oral ingestion is also relevant in the occupational setting. Most of the inhaled nanomaterials are trapped in the respiratory system and are transported upward via the "mucociliary elevator" and finally swallowed down or coughed out. Nanomaterials that enter the GI tract are immediately exposed to saliva. Thereafter, they will be transported into the stomach where they are exposed to its harsh conditions (the $\mathrm{pH}$ of gastric acid whose main constituent is hydrochloric acid is between 1.5 and 3.5) prior to being transferred into the small and large intestines, where nutrients are resorbed out of the bolus. Several factors such as digestive enzymes, $\mathrm{pH}$, ionic strength, surface-active compounds, and type and amount of food intake have the potential to change the physicochemical properties of nanomaterials, which has to be taken into account in the hazard assessment of nanomaterials following the oral exposure route. ${ }^{180}$ The small intestine with its villous structure provides a large mucus-protected surface of around $2000 \mathrm{~m}^{2}$, the largest in the human body, enabling efficient nutrient uptake. The intestinal epithelium is composed mainly of enterocytes, mucus-producing goblet cells, and so-called microfold cells 
( $M$ cells) important for the induction of an efficient immune response. ${ }^{182}$ The latter cells initiate mucosal immunity responses on the apical membrane and allow for transport of microbes and particles across the epithelial cell layer from the gut lumen to the lamina propria, where interactions with immune cells take place. ${ }^{183}$ The potential uptake and translocation routes of particles across the GI barrier could be either paracellular or transcellular pathways. In addition to the cellular barrier, the mucus lining of the GI tract forms an important and effective biological barrier against nanoparticle uptake and translocation into the systemic circulation. ${ }^{184}$ It is important to elucidate how GBMs interact with the GI system in comparison to other particles in order to estimate the health risks of this class of materials, but few studies are available to date.

One of the most commonly accepted in vitro intestinal models in pharmaceutical and toxicological research consists of the human colon adenocarcinoma cell line, Caco-2. This cell line can be maintained as subconfluent cultures representing preenterocytes. However, after 3 weeks of cultivation, the cells fully differentiate to enterocytes, undergoing intense morphological and physiological changes such as polarization, formation of microvilli structures, and changes in gene and protein expression compared to nondifferentiated cells. ${ }^{185} \mathrm{Caco}-2$ cells have been used in numerous studies to evaluate the potential impact of nanoparticles on the GI tract. ${ }^{186-188}$ In recent years, more advanced in vitro models have been developed such as $3 \mathrm{D}$ cocultures, ${ }^{189}$ gut organoids or "mini-guts", ${ }^{190}$ or gut-on-a-chip models. ${ }^{191}$ However, there are few if any studies on the effects of GBMs using such models. Nevertheless, some recent studies have addressed the potential toxicity of GBMs using the Caco-2 cell model (supporting Figure S5). Nguyen et al. exposed Caco-2 cells to GO flakes at different concentration $(10-500 \mu \mathrm{g} / \mathrm{mL})$ and observed only mild cytotoxic effects at higher concentrations. ${ }^{192}$ They speculated that the adsorption of medium nutrients to the flakes might be responsible for this observed effect. In a recent study conducted in the Graphene Flagship, the uptake of GBMs was shown to be strongly dependent on the differentiation state of the cells. ${ }^{81}$ Nondifferentiated Caco- 2 cells were able to incorporate GO and GNP agglomerates of several micrometers in size in a dose-dependent manner, whereas differentiated Caco- 2 cells displayed repellent properties toward GBMs due to the presence of densely packed microvilli. This suggests that the choice of in vitro models is crucial for the outcome of the study. Ruiz et al. performed a test of the role of GO-coated surfaces on mammalian cell attachment and proliferation. ${ }^{193}$ To this end, control glass slides and glass slides coated with GO produced by Hummers' method were placed onto a culture dish to which the colorectal adenocarcinoma HT-29 cells were added. The results showed that GO film coated on glass slides enhanced cell attachment, growth, and proliferation of these cells. In order to further elucidate the influence of the physicochemical properties of GBMs on Caco-2 responses, Kucki et al. investigated a panel of GBMs (i.e., four GOs and one GNP). ${ }^{194}$ The GOs differed in the following parameters: (i) size (from a few hundred nanometers to several micrometers in lateral dimensions), (ii) starting material (graphite versus graphite nanofiber), (iii) C/O ratio (around 2 for the GO samples and 24 for GNP), and (iv) number of layers (thickness: $1 \mathrm{~nm}$ to $5 \mu \mathrm{m}$ ). The main outcome of this study was that all four GOs were noncytotoxic toward nonconfluent Caco-2 cells, which are considered to be more sensitive than the differentiated cells. Only relatively high concentrations (up to $80 \mu \mathrm{g} / \mathrm{mL}$ ) induced a response. Pretreatment of the materials with acid to mimic the conditions in the GI tract did not influence the outcome. The GNP aggregates, on the other hand, yielded a low level of acute toxicity at high concentrations, indicating that aggregation, number of layers, or $\mathrm{C} / \mathrm{O}$ ratio have a more pronounced effect on cell viability than the lateral dimensions alone. ${ }^{194}$ In another related study, treatment of GO and FLG with digestive juices to simulate oral ingestion did not induce structural changes/degradation of the materials, and chronic exposure to the digested GBMs did not affect the intestinal Caco-2 barrier despite long-term exposure $(1$ and $5 \mu \mathrm{g} / \mathrm{mL}$; 2 h every 2 days up to 9 days). ${ }^{195}$

The microbiome is considered to be our "forgotten organ," and the gut microbiota is involved in the regulation of multiple metabolic, signaling, and immune-inflammatory pathways in the host that physiologically connect the gut, liver, muscle, and brain. ${ }^{196}$ In a recent in vivo study, GO was found to exert milder effects following oral exposure when compared to single-walled carbon nanotubes (SWCNTs) and multiwalled carbon nanotubes (MWCNTs). ${ }^{197}$ Importantly, the authors also investigated whether these carbon-based nanomaterials had any impact on the gut microbiome by assessing the impact on gut microbiota composition using 16S rRNA gene sequencing approaches. Analysis of the microbiota at various taxonomic levels showed marked changes of diversity and composition of gut microbiota after acute oral administration of SWCNTs, MWCNTs, and GO. The 16S rRNA gene sequencing results showed significant shifts of the predominant microbe phyla from Firmicutes to Bacteroidetes in SWCNT-treated mice, and exposure to MWCNTs yielded similar alterations as noted for SWCNTs at both phyla and genus levels. ${ }^{197}$ However, compared to the oral exposure to SWCNTs and MWCNTs, the exposure to GO showed a more pronounced impact on the gut microbiota (Figure 5).

In conclusion, the first explorative studies in this relatively new field have shown no or only mild acute cytotoxicity of several different GBMs on intestinal epithelial cells. However, the field is in its infancy, and aspects of long-term effects of GBMs including the influence on the microbiota remain unanswered today. Therefore, further studies are needed to understand the potential impact of different GBMs following oral exposure.

Reproductive and Developmental Effects of GrapheneBased Materials. Pregnant women, fetuses, and neonates are among the most vulnerable populations and therefore warrant particular attention in regard to GBM hazard assessment. In pregnancy, major physiological changes occur that are expected to affect particokinetics and subsequent biological effects. Likewise, developing fetuses and neonates are more susceptible to toxic effects of xenobiotics than adults due to ongoing organogenesis, physiological changes, or immaturity of the immune system. To date, it is unknown whether GBMs can reach the placental barrier or reproductive organs. The low transfer of nanoparticles at the air-lung, skin, and intestinal barrier $^{198}$ and the rapid clearance of GBMs from the bloodstream (discussed above) would argue for a low acute exposure under currently prevailing inhalation and oral exposure scenarios. However, emerging biomedical applications of GBMs and the possibility of tissue accumulation upon chronic exposure of these materials clearly suggests that close attention is needed to the potential reproductive and developmental risks of GBMs.

First indications that nanoparticles may interfere with pregnancy and fetal health came from epidemiological studies showing that maternal exposure to air pollution (in particular, to particulate matter $<2.5 \mu \mathrm{m}$ ) during pregnancy was associated 
Control

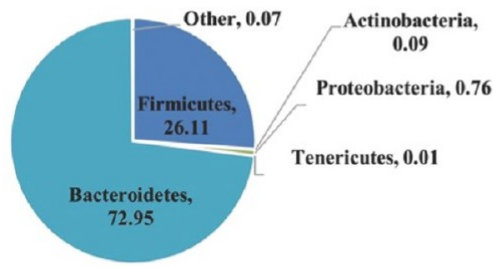

SWCNTs

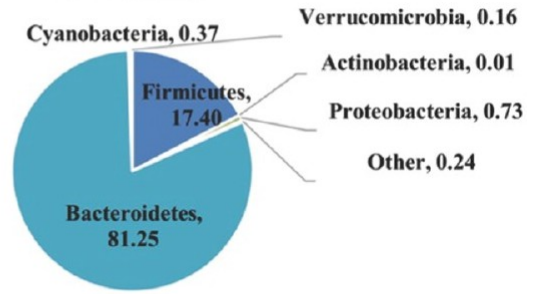

MWCNTs

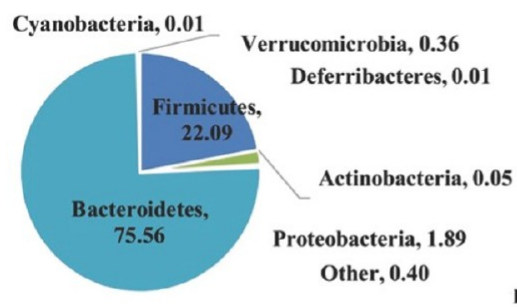

GO

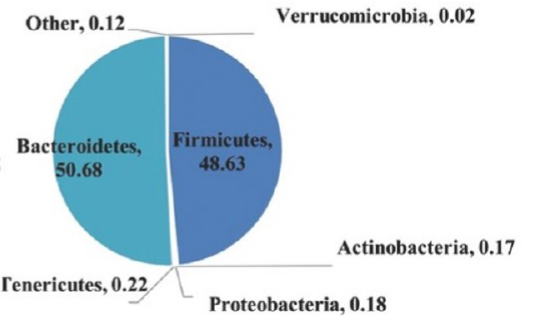

Figure 5. Carbon-based nanomaterials and the gut microbiome. Comparison of bacterial community abundance at phylum level after acute oral administration of SWCNTs, MWCNTs, and GO to mice $(2.5 \mathrm{mg} / \mathrm{kg}$ for 7 days). The pie charts show the relative abundance of gut microbiota based on 16S rRNA gene sequencing. Reprinted with permission from ref 197. Copyright 2018 Wiley-VCH Verlag GmbH \& Co.

with adverse birth outcomes such as low birth weight and preterm birth (reviewed in ref 199). As a consequence, research on the placental transfer of nanoparticles and the impact on reproductive and developmental systems was intensified. For carbonaceous nanomaterials including carbon nanotubes, the findings suggest that these materials may, indeed, have the potential to adversely affect pregnancy and embryonic/fetal development. ${ }^{200}$ However, for GBMs, the existing literature is still too limited, and results are too conflicting to draw firm conclusions regarding their potential reproductive and developmental risks. Male fertility and reproduction was not affected after intravenous and intraperitoneal injection of small or large GO in mice ${ }^{201}$ nor by pulmonary exposure to GO. ${ }^{202}$ Moreover, no damage to testis tissue was apparent in male mice after intravenous injection of $\mathrm{GO},{ }^{89} \mathrm{FLG}$, oxidized FLG, or PEGylated FLG. ${ }^{93}$ In addition, pre- or postfertilization injection of singleor few-layer small (20-150 nm) and large (200-1500 nm) rGO did not alter sex hormone levels in female mice. ${ }^{203}$ However, in the latter study, the authors observed that when small rGO was injected in late gestation, this resulted in abortions, malformed fetuses, and death of pregnant mice. Thus, based on these observations, the toxicity of rGO should be seriously considered in progestational (drawing near pregnancy) females, although the rGO-exposed mice could still produce healthy offspring depending on the administered dose. ${ }^{203}$ Developmental toxicity of GBMs has also been described in other species including zebrafish and chicken, ${ }^{204,205}$ but because these models lack a mammalian maternal-placental-embryonic/fetal relationship, their predictive value for human developmental and reproductive toxicity assessment is limited.

The placenta forms the interface between mother and fetus and enables successful pregnancy by mediating essential functions including exchange of gases, nutrients, and waste products, hormone secretion, feto-maternal immune tolerance, and fetal protection against pathogens and xenobiotics. Therefore, it is critical to understand placental translocation and effects of GBMs in order to estimate their embryo fetotoxic risks. However, it is currently unclear if and by which pathway(s) GBMs may pass the placental barrier at different stages of pregnancy.
A single study investigating transplacental transfer of ${ }^{125} \mathrm{I}-\mathrm{rGO}$ after intravenous injection in pregnant mice in late gestation measured only trace amounts of radioactivity in the placental or fetal tissues (approximately $0.3 \%$ of the applied dose), which may also have resulted from transfer of free ${ }^{125} \mathrm{I}^{205}$ Nevertheless, placental translocation has been described for different nanoparticles throughout pregnancy including carbonaceous materials such as MWCNTs. ${ }^{206-208}$ Interestingly, embryo fetotoxic effects of carbon nanomaterials were not necessarily correlated with placental transfer of nanoparticles (direct effects) but may result from adverse effects of particles on maternal and placental tissue (indirect effects). ${ }^{209,210}$ For small rGO, malformed fetuses and abortions were found after injection during late gestation without apparent particle translocation. ${ }^{205}$ The authors suggested that adverse effects elicited by small rGO in the maternal mice (e.g., decrease of white blood cell number) may indirectly account for the observed developmental toxicity. Another example of a maternally mediated effect was a reduced growth of the offspring when maternal mice were given GO-containing drinking water $(0.5 \mathrm{mg} / \mathrm{mL}) .{ }^{211}$ Potential placenta-mediated effects of GBMs, e.g., interference with placental viability and functionality, have not been extensively explored. However, in a recent study in the Graphene Flagship, the impact of four GO samples on human BeWo trophoblast cells did not reveal overt cytotoxicity after $48 \mathrm{~h}$ of exposure at concentrations up to $40 \mu \mathrm{g} / \mathrm{mL}$ despite internalization of the GO sheets. ${ }^{212}$ On the other hand, exposure to GO induced a transient opening of the trophoblast barrier as evidenced by a temporary increase in the translocation of sodium fluorescein and a slight decrease in human choriogonadotropin secretion. ${ }^{212}$ These observations underscore the need for further studies on the long-term consequences of GBMs on placenta functionality and maternal-fetal health. It is pertinent to note that rGO has been suggested to induce a transitory decrease in the tightness of the blood-brain barrier in rats; rGO was systemically injected in the latter study, and the relatively large size (average size: $342 \mathrm{~nm}$ ) of the material was apparently not an obstacle for its entry into the brain. ${ }^{213}$ Overall, a better understanding of potential interferences of GBMs with placental, reproductive, and developmental functions will be 
imperative for the sustainable and safe use of GBMs, not least as reproductive and developmental toxicity has been reported for other carbon-based nanomaterials. ${ }^{209,214}$ For studies on placental translocation and effects of GBMs, human models (e.g., ex vivo placenta perfusion, placental explant cultures, or placental microtissues) are available to complement in vivo studies and to avoid uncertainties in the extrapolation of results due to species-specific differences in placental structure and function. ${ }^{215}$

Finally, in addition to direct effects of GBMs on reproductive and developmental systems, the indirect consequences of GBMs on maternal and placental tissues and the release of mediators deserves attention as the creation of a hostile environment in the womb may increase the risk for pregnancy complications and the development of diseases later in life.

Central Nervous System Effects of Graphene-Based Materials. Graphene holds exciting prospects in neuroscience; the unique physicochemical properties, such as the high conductivity, transparency, or flexibility, make this material an attractive candidate to engineer functional brain implants with excellent performance for neuromodulation therapies or to design scaffolds able to support the reconstruction of functional neurons and glial cells networks, also an imperative requirement for neural regeneration of central nervous system (CNS) injuries. ${ }^{216}$ Particularly relevant in neurology is the on-demand release of drugs enabling precise targeted dosing to meet the requirements of diverse therapeutic applications. Naturally, the implementation of multifunctional neurodevices based on graphene will expose brain cells and neuronal circuits directly to this material by injection or implantation, and safety assessment of graphene and its derivatives is therefore of paramount importance. In the following sections, we will discuss interactions of GBMs with the CNS.

Studies conducted in the Graphene Flagship have disclosed that GBMs are able to interact with and perturb cells of the CNS in different ways, as a function of their intrinsic characteristics. ${ }^{217,218}$ Hence, investigations exploring the responses of brain cells to prolonged GO exposure, pointed out a clear lateral size-related cytotoxicity. ${ }^{217}$ The effect of GO flakes of differing lateral dimensions was evaluated on cells belonging to relevant structures of the CNS, maintained in culture. Neurons and glial cells from dissociated rat hippocampus or cortex were cultured in the presence of $10 \mu \mathrm{g} / \mathrm{mL}$ dispersions of large and small GO. ${ }^{217}$ After 6-8 days of incubation, it appears that large, micrometersized flakes of GO induced unequivocal neuroglial and neuronal loss. Interestingly, when cells were treated with the same concentration of FLG, no reduction in cell density or viability was observed in both neuronal and glial populations, thus demonstrating that CNS cells survival in vitro seems crucially dependent on the graphene sheet dimensions as well as its chemical composition. ${ }^{217}$ However, even in the absence of cytotoxicity, we cannot rule out other potential effects on neuronal or glial function brought about by the exposure to GBMs. ${ }^{217}$ In these experiments, patch clamp recordings and fluorescence imaging were used to check the ability of FLG and GO nanosheets (about $100 \mathrm{~nm}$ in lateral dimensions) to interfere with synaptic signaling when cells were exposed for 1 week to a growth medium containing such materials at 1 or $10 \mu \mathrm{g} / \mathrm{mL}$ concentrations. Passive cell properties, neuronal network organization, and overall network activity of neurons interfaced with FLG at both concentrations did not differ from control hippocampal cells. Instead, small GO flakes interfered specifically with neuronal synapses, albeit without affecting cell viability. ${ }^{217}$ In particular, while at the lower concentration of GO, network synaptic activity was not altered, a significant reduction in postsynaptic current frequency was detected at $10 \mu \mathrm{g} / \mathrm{mL}$. Thus, GO nanoflakes at higher concentrations seem able to specifically downregulate synaptic activity. The results also showed that GO nanoflakes only impaired excitatory (glutamate AMPA receptormediated) synapses, whereas they did not impair inhibitory $\mathrm{GABA}_{\mathrm{A}}$-mediated connections. ${ }^{217}$ The authors proposed that the selective interference of GO with the excitatory presynaptic terminals versus the inhibitory GABAergic terminals could be due to the different dimensions of the excitatory and inhibitory synaptic clefts. ${ }^{219}$ On the other hand, the different behavior of FLG of matching dimensions could be explained by their hydrophobic surface resulting in a modest ability to interact with the plasma membrane as the formation of aggregates in the cell culture are not suited to interface with submicroscopic structures such as synapses in the brain.

Studies were also conducted to test the ability of nanosized FLG and GO to reduce exocytosis and recycling of synaptic-like microvesicles from cultured primary glial cells. ${ }^{217}$ Microvesicles are released into the extracellular space by direct budding from the plasma membrane of astrocytes and have been shown to have an important role in intercellular communication. ${ }^{220}$ Pure glial cell cultures were treated with FLG and GO suspensions $(10 \mu \mathrm{g} / \mathrm{mL})$ for $6-8$ days. FLG and GO did not affect astrocyte density, excluding a cytotoxic effect. It is well-known that microvesicle release could be artificially induced in glial cells cultures by $2^{\prime}, 3^{\prime}$-[benzoyl-4-benzoyl]-ATP (BzATP) and subsequently detected and quantified by immunoblot analysis of the collected supernatant. $^{221}$ Surprisingly, the treatment of astrocytes with GO resulted in an effect similar to BzATP stimulation inducing pronounced vesicle release. Intriguingly, similar experiments with FLG at the same concentration and duration did not induce shedding of microvesicles in glial cell cultures. Summarizing the results obtained thus far, one can conclude that while the lateral size of graphene flakes is critical in defining material cytotoxicity, the oxidative state also plays a role and may explain the difference between a neutral effect of FLG versus the ability of GO to perturb innate vesicular regulation mechanisms presumably via plasma membrane interactions.

The physical interaction of GBMs with the plasma membrane is strongly affected by the physicochemical properties of the material. Recent studies showed that FLG and GO nanosheets were internalized by neurons mainly through the endosomal/ lysosomal pathway; moreover, electron microscopy analysis revealed a number of particles free in the cytoplasm, which had either pierced the membrane or escaped from intracellular organelles (Figure 6). ${ }^{218}$ Of note, no particles were observed inside the nucleus, either in neurons or in astrocytes, thus making it unlikely for any direct genotoxic damage to occur. ${ }^{218}$ The amount of internalized material (FLG and GO) was relatively low for neurons and never exceeded $15 \%$ of the total amount of material present in the cell culture, whereas astrocytes and microglia internalized up to $30-40 \%$ of the administered flakes, coherent with their primary function in defending neurons from insults. ${ }^{218}$ Furthermore, whereas nanosheet-exposed neurons formed a well interconnected network, astrocytes displayed marked morphological alterations, reminiscent of activated/ mature glia, and similar to those changes induced by carbon nanotubes. ${ }^{222}$ Such morphological changes are likely due to nanomaterial interactions with and disruption of the cellular actin cytoskeleton. $^{223,224}$

Furthermore, and in contrast to the aforementioned studies in which cells were exposed to nano- or micron-sized flakes, studies on surface-immobilized GBMs have also been reported. Tu et al. 

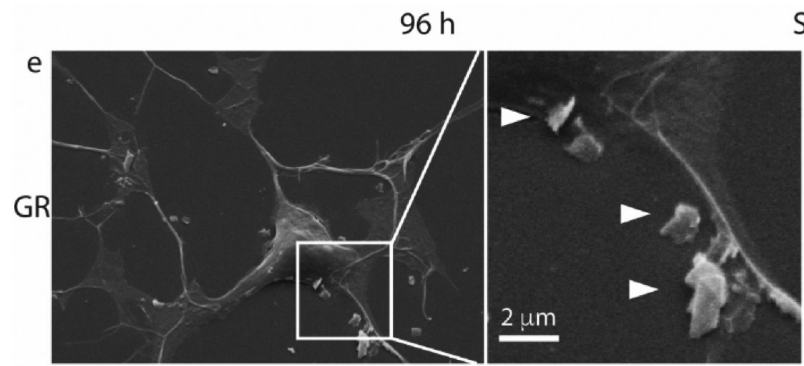

SEM $14 \mathrm{~d}$
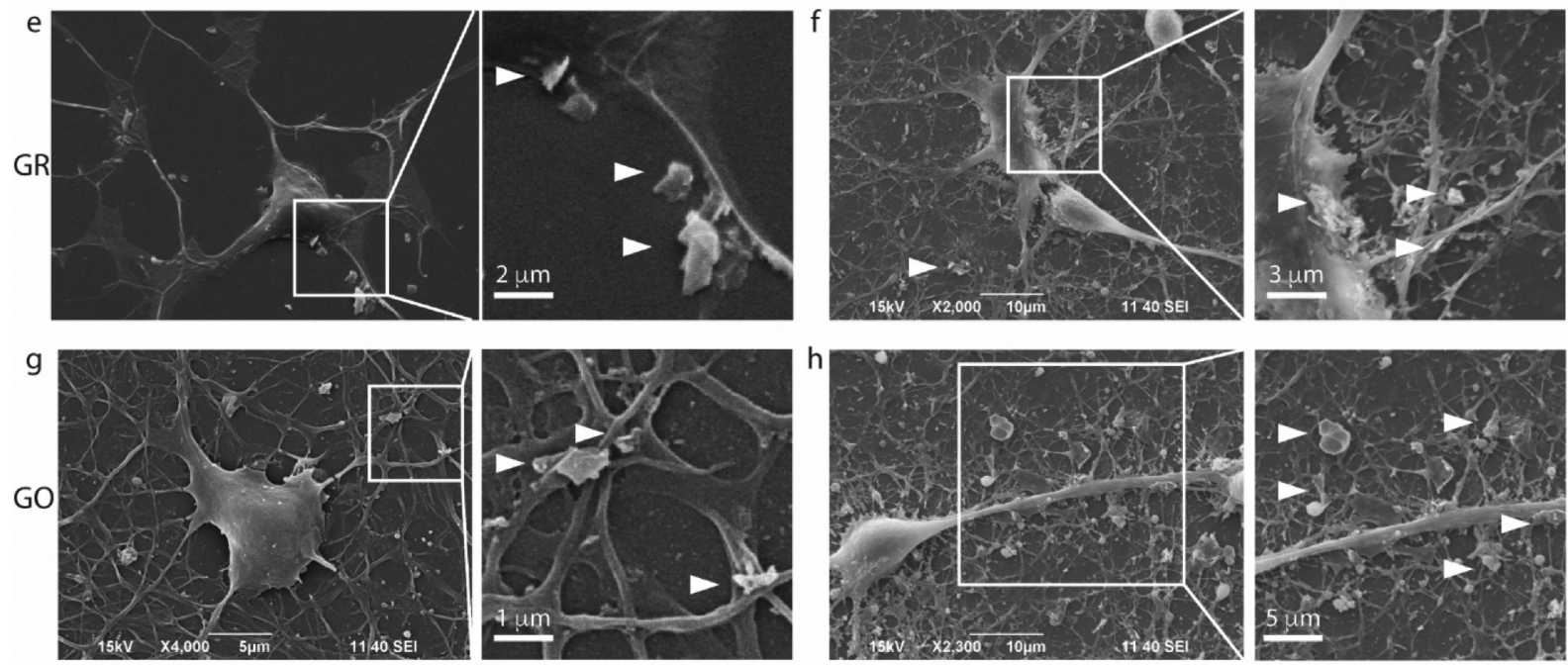

$96 \mathrm{~h}$
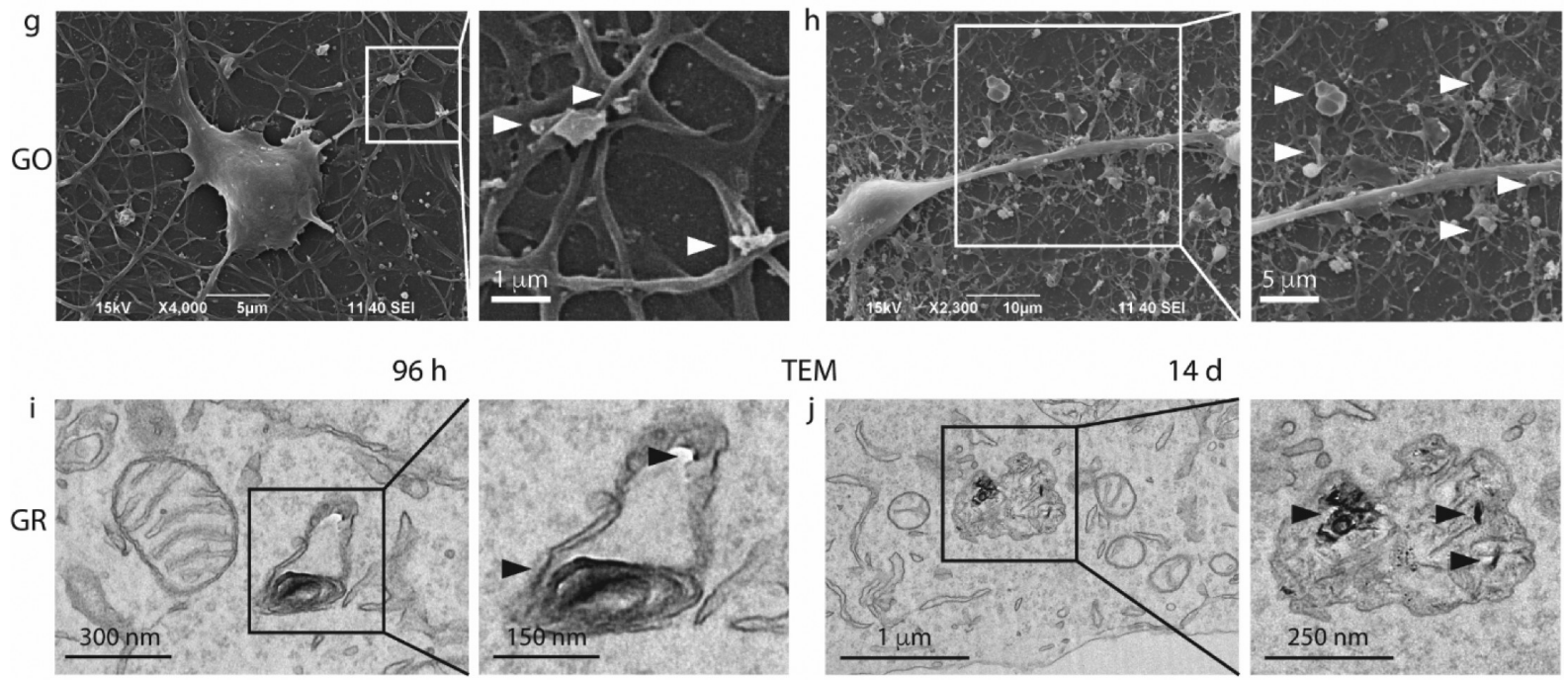

TEM

$14 \mathrm{~d}$
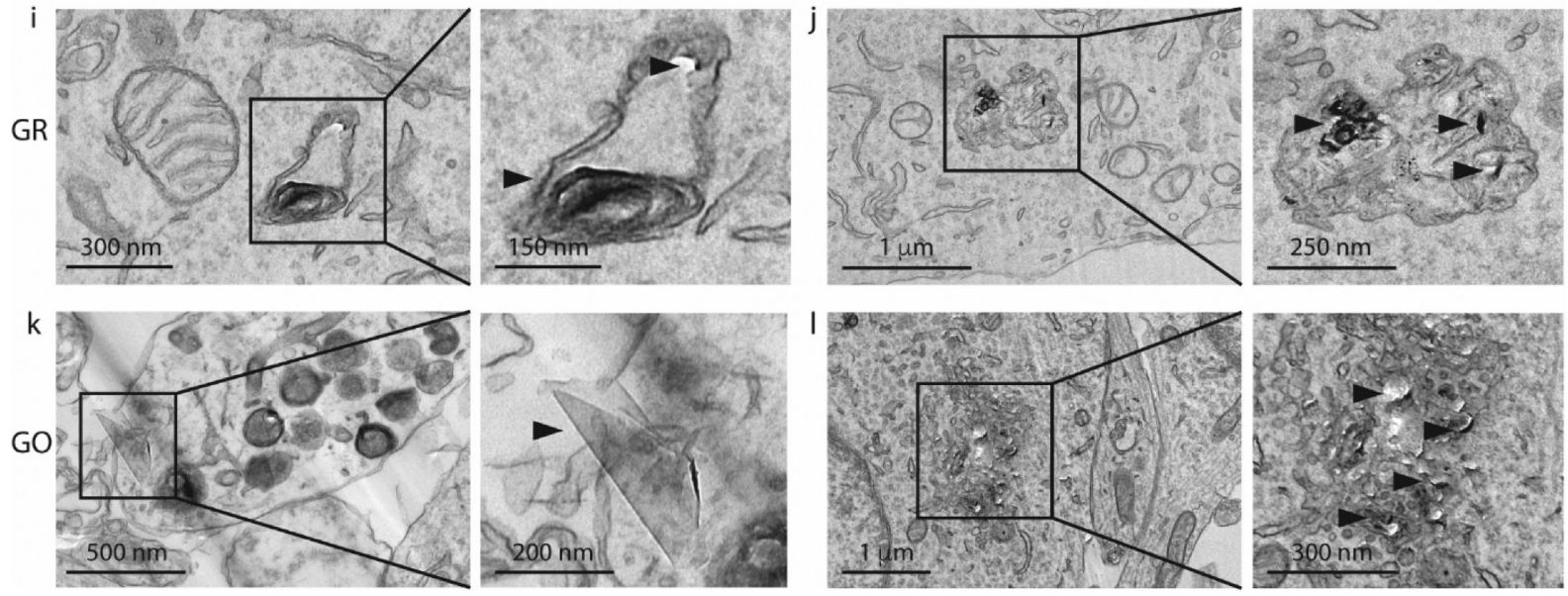

Figure 6. Graphene interactions with neurons. Primary rat cortical neurons were exposed to FLG (here abbreviated GR) and GO flakes (1 and $10 \mu \mathrm{g} / \mathrm{mL}$ ) for $96 \mathrm{~h}$ or 14 days or to equivalent volumes of the respective vehicles. SEM was used to study the interaction of flakes with neuronal cells. (e-h) Large number of flakes (white arrowheads) were found in contact with the cell membrane; however, cell morphology and network development were largely unaffected. (i-l) Cell uptake of FLG and GO and intracellular localization were studied by TEM. At $24 \mathrm{~h}$, most of the flakes were found outside the cells (not shown). However, starting from $96 \mathrm{~h}$, flakes were internalized into intracellular vesicles (i,j; black arrowheads) or free in the cytoplasm (1; black arrowheads). Reprinted from ref 218. Copyright 2016 American Chemical Society.

systematically modified the properties of GO by attaching different functional groups and found that by manipulating the charge carried by the functionalized GO, the outgrowth and branching of neuronal processes could be controlled. ${ }^{25}$ Thus, compared with neutral, zwitterionic, or negatively charged GO, positively charged GO was found to be more beneficial for neurite outgrowth and branching in a model of primary rat hippocampal neurons grown on GO-coated glass slides. Recent studies from the Graphene Flagship focused on surface-immobilized graphene produced by liquid phase exfoliation or ball-milling of graphite and showed that such substrates are inert neuroninterfacing materials, able to preserve the basal physiological level of neuronal activity. ${ }^{226}$ Hence, graphene-based substrates were successfully used to support the development of primary neurons from rat hippocampus. In a related study, changes in membrane cholesterol were noted in hippocampal neurons grown on graphene-coated surfaces, resulting in a presynaptic potentiation of neurotransmission. ${ }^{27}$ More recently, another study from the Graphene Flagship showed that single-layer graphene increases neuronal firing of rat hippocampal neurons by tuning the distribution of extracellular ions at the neurongraphene interface (Figure 7). ${ }^{228}$ This work hypothesizes, on the basis of experimental and theoretical approaches, that this is due to the interactions between graphene and cations, in particular, potassium, that are maximized when graphene is deposited on electrically insulating substrates. ${ }^{228}$ The possibility arises to exploit such substrates as next generation brain interfaces. In this context, the uncommon ability of surfaces decorated by immobilized graphene to support neuronal development (in terms of neuronal passive properties, spontaneous synaptic activity, synaptogenesis, and short-term synaptic plasticity) without precoating with adhesion-promoting peptides (e.g., polylysine or polyornithine) deserves to be highlighted. Previous work demonstrated the biocompatibility of peptide-coated chemical vapor deposited graphene interfaces with hippocampal neurons (polylysinecoated graphene $)^{229}$ or neural stem cells (laminin-coated graphene). ${ }^{230}$ However, peptide coating might weaken neuron/ interface electrical contacts and electrical signal transmission, resulting in non-optimal charge transfer. ${ }^{231,232}$ Surface immobilization of graphene seems to prevent toxic effects and could be 

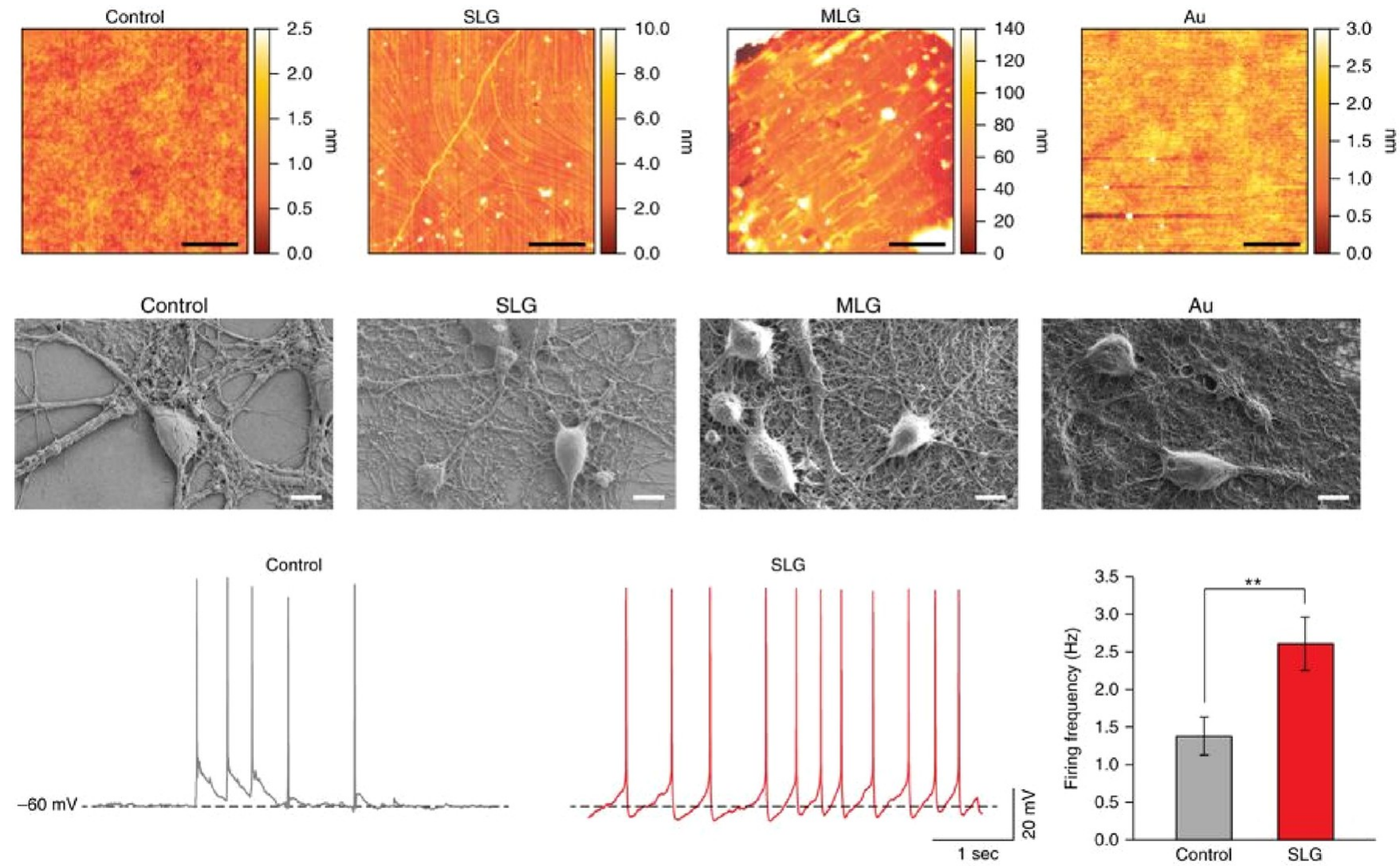

Figure 7. Graphene modulation of neuronal communication. (Top row) AFM topography reconstructions of glass control, single-layer graphene (SLG), multilayer graphene (MLG), and gold-plated glass surfaces. AFM documented a surface roughness of the materials that varied from $0.23 \pm 0.02 \mathrm{~nm}$ for the control $(n=3), 1.5 \pm 0.5 \mathrm{~nm}$ for SLG $(n=3), 20 \pm 10 \mathrm{~nm}$ for MLG $(n=3)$, and $0.47 \pm 0.1 \mathrm{~nm}$ for Au $(n=3)$. Scale bar, $5 \mu \mathrm{m}$. (Middle row) Representative SEM images depicting hippocampal neuron morphology after 10 days in vitro, supported by the different substrates. Culture substrates were not pretreated with additional adhesion molecules that might mask the effects of graphene. Scale bar, $10 \mu \mathrm{m}$. (Bottom row) SLG triggers changes in single-cell intrinsic excitability. Representative current-clamp recordings of hippocampal neurons in culture for 10 days. Control and SLG neurons displayed similar resting membrane potentials $(-52 \pm 10 \mathrm{mV}$ in SLG; $-50 \pm 7 \mathrm{mV}$ in control). When maintained at $-60 \mathrm{mV}$, the spontaneous action potential firing was measured as summarized in the histograms (right). Note the significantly higher action potential frequency in SLG $(2.60 \pm 0.36 \mathrm{~Hz}$ in neurons grown on SLG, $n=21 ; 1.37 \pm 0.26 \mathrm{~Hz}$ in control, $n=19 ; P=$ $0.0054) . * P<0.05, * * P<0.01$. Reprinted with permission from ref 228 . Copyright 2018 Nature Publishing Group.

exploited to promote neuronal development. However, more studies are needed to evaluate the long-term integrity of such substrates. Furthermore, although studies on explanted neurons are informative, detailed in vivo studies on the influence of graphene on neuronal microcircuits are lacking.

For a comprehensive view of the impact of GBMs on the brain, it is important to address not only effects on neurons but also effects on non-neuronal cells, i.e., glial cells including astrocytes and microglia. To better understand the molecular and cellular processes affected by the exposure to GBMs, proteomic and lipidomic analyses were conducted on primary neuron and astrocyte cultures exposed to GO and, in the case of astrocytes, to GO or FLG. ${ }^{218}$ Among the common pathways affected in both neurons and glial cells, we find $\mathrm{Ca}^{2+}$ signaling, of vital importance in almost every aspect of neural cell physiology, with several $\mathrm{Ca}^{2+}$-binding and buffering proteins being markedly up- or downregulated in exposed cultures, along with intracellular trafficking, which likely mediates the observed endocytotic and/or phagocytic responses. ${ }^{218}$ The lipidomics analysis revealed that the exposed neurons were characterized by an upregulation of phosphatidylethanolamine and downregulation of phosphatidylserine. $\mathrm{PE}$ is one of the major components of the plasma membrane and synaptic vesicle membrane and plays important roles in vesicle fusion and fission. ${ }^{233}$ Cholesterol was found to be one of the most altered lipids in astrocytes exposed to the nanosheets. ${ }^{218}$ Cholesterol is a structural component of lipid rafts, which mediate the signaling between endoplasmic reticulum and plasma membrane in astroglial cells. ${ }^{234}$ Regarding the functionality of nanosheet-exposed cultures, a closer analysis of $\mathrm{Ca}^{2+}$ dynamics revealed marked alterations in both neurons and astrocytes consisting of reduced number of spontaneously oscillating cells, reduced basal cytoplasmic $\mathrm{Ca}^{2+}$ concentration, and altered responses to external stimuli. Interestingly, these effects were elicited only by chronic GO exposure, whereas acute exposure to FLG and GO did not cause any functional alterations in both culture systems. For astrocytes, recent studies showed that a marked alteration of $\mathrm{K}^{+}$currents was selectively triggered by GO. ${ }^{235}$ More specifically, an increase in outward rectifying currents was observed, together with a hyperpolarized membrane potential, decreased input resistance, and increased specific conductance. Interestingly, a significant increase in astrocyte-released microvesicles was also observed in cell cultures treated with GO. ${ }^{217} \mathrm{Ca}^{2+}$ dynamics, glutamate uptake, and microvesicle release are all fundamental processes in the astrocyte-to-neuron communication. To conclude, in vitro studies on primary neurons and glial cells show that, while chronic 
exposure to FLG or GO does not cause cell death, it has a strong impact on a number of fundamental physiological processes, thus potentially leading to toxicity when administered for prolonged amounts of time. In fact, studies performed in the Graphene Flagship have revealed a size-dependent toxicity of graphene toward neurons and glial cells. However, some characteristics of FLG could potentially be harnessed to restore pathological alterations in the CNS. Thus, future studies should address the possibility of functionalizing GBMs to exploit selected features while tuning properties that could potentially lead to unwanted effects.

\section{ENVIRONMENTAL HAZARD ASSESSMENT OF GRAPHENE-BASED MATERIALS}

The tremendous advancement in the field of nanotechnology has been accompanied by a slower progress in the understanding of its impact on the environment. Large-scale production, leaching out of from enriched products, accidental spills during industrial production, and poor disposal of the derived wastes might result in significant release and accumulation of GBMs in the environment. This phenomenon already occurred for other synthetic materials such as plastics that were hailed as the "discovery of the century" by their inventors and producers and correctly predicted to change the everyday lives of people. However, although the promises were fulfilled, the benefits were unfortunately counterbalanced by unexpected environmental problems, which emerged in dramatic fashion only half a century later. ${ }^{236}$ Hence, exploring the ecotoxicity of GBMs is of fundamental importance. ${ }^{237}$ To this end, a wide range of organisms has been investigated, not least in the Graphene Flagship, including bacteria, algae, seed plants, invertebrates, and vertebrates in a variety of ecosystems.

Effects of Graphene-Based Materials on Bacteria. Studying the effects of carbon-based nanomaterials on bacteria is essential as they are at the basis of the trophic chains in the environment, involved in many stages of the nutrient cycles, and have complex associations with other organisms. Effects of GBMs on bacteria are rather well-studied, compared to other living systems. The most commonly used laboratory model is Escherichia coli. Several studies have shown that direct contact between GBM and bacteria is responsible for the observed toxicity toward E. coli and other bacteria. ${ }^{238-244}$ Liu et al. described the antibacterial activity of four types of GBMs (graphite, graphite oxide, GO, and rGO) toward E. coli. GO dispersions exhibited the highest antibacterial activity, followed by rGO, graphite, and graphite oxide. ${ }^{238}$ Dizaj et al. reported that the physical interaction between microorganisms and carbonbased nanomaterials (carbon nanotubes, GO, and fullerene) affected cellular membrane integrity, metabolic processes, and morphology of microorganisms. ${ }^{239}$ Using experimental and theoretical (modeling) approaches, $\mathrm{Tu}$ et al. suggested that graphene and GO nanosheets can induce the degradation of the inner and outer cell membranes of E. coli, thereby reducing their viability. ${ }^{245}$ In a more recent study designed to address the role of functional groups, Li et al. utilized reduction and hydration methods to establish a GO library with different oxidation, hydroxyl, and carbon radical $\left(\mathrm{C}^{\bullet}\right)$ levels to study the impact on antibacterial activity. ${ }^{246}$ Using antibiotic-resistant bacteria, the authors could show hydrated GO, with the highest $\mathrm{C}^{\bullet}$ density, had the strongest antibacterial effects through membrane binding and induction of lipid peroxidation, suggesting that $\mathrm{C}^{\bullet}$ is the principle source that can be utilized for clinical applications of GO-based antibacterial coatings, e.g., catheters. On the other hand, Ruiz et al. reported that GO-coated surfaces could promote proliferation of $E$. coli with the formation of dense biofilms. ${ }^{193}$ Furthermore, Guo et al. observed that GO significantly enhanced the cell growth, biofilm formation, and biofilm development for E. coli and Staphylococcus aureus, whereas rGO strongly inhibited cell growth and biofilm formation. ${ }^{243}$ To investigate the orientation-dependent interaction of GBMs with bacteria, Lu et al. aligned GO nanosheets in a magnetic field, immobilized by cross-linking of the surrounding matrix, and exposed on the surface through oxidative etching. ${ }^{247}$ The GO nanosheets with vertical orientation exhibited enhanced antibacterial activity toward E. coli compared with random and horizontal orientations of GO. The authors proposed that the antibacterial mechanism requires penetration of the cell membrane, suggesting that the enhanced antibacterial activity of the film with vertically aligned GO is due to an increased density of edges with an orientation that is more compatible with membrane disruption. ${ }^{247}$ In another recent study, graphene flakes grown perpendicularly to the surface exhibited a strong inhibitory effect on the adhesion of biofilms of E. coli and $S$. epidermidis, causative agents of urinary tract infections and infections related to implants and catheters. ${ }^{248}$ The authors also reported that this graphene-based "bed-of-nails" did not impart any cytotoxicity toward murine fibroblasts or human neuroblastoma cells. Thus, the properties of GBMs as well as their orientation and the degree of membrane interactions control their antibacterial effects.

Effects of Graphene-Based Materials on Photoautotrophs. Recently, the ecotoxicity of GBMs was evaluated on various model and nonmodel photoautotrophs, from cyanobacteria to seed plants. These organisms are all characterized by the presence of a cell wall of different composition and ultrastructure (peptidoglycans in cyanobacteria, cellulose in algae, and embryophytes), often completed by further external structures (in cyanobacteria, an outer lipopolysaccharidic membrane, a capsule, and a gelatinous sheath; in many algae, a layer of exopolymeric substances). The cell wall is a physical barrier that retards the entrance of GBMs larger than the pore size of the cell wall. ${ }^{249}$ Moreover, the interaction could differ not only among organisms but also with the age of the organism. In the cells of seed plants, for instance, thickness and complexity of cell wall change drastically from the pectic-rich primary wall, generally a thin, flexible, and extensible layer formed when the cell is growing, to the thick secondary wall, formed after the cell is fully grown, made of cellulose, xylan, and lignin, which strengthens and waterproofs the wall. Observations on GBM internalization carried out on cell cultures in active division can thus produce divergent results in comparison to studies based on adult tissues or organs. Not surprisingly, internalization has been repeatedly reported in tobacco cell cultures and in a few thin-walled green algae, such as Chlorella pyrenoidosa and C. vulgaris, but could not be confirmed in the thick-walled green alga, Trebouxia gelatinosa (see below).

Despite their considerable ecological importance, cyanobacteria have rarely been studied in relation to ecotoxicology of GBMs. The freshwater Microcystis aeruginosa was investigated by Tang et al., who tested combined exposures to GO and $\mathrm{Cd}^{2+}$ (concentrations between $1-50 \mu \mathrm{g} / \mathrm{mL}$ and $0.2-0.7 \mu \mathrm{g} / \mathrm{mL}$, respectively). ${ }^{250}$ The authors observed that GO alone at low concentrations had no significant toxicity, even if the material easily adhered to and entered into the algal cells. However, mortality and induction of oxidative stress due to $\mathrm{Cd}^{2+}$ uptake were both increased by the presence of GO. Furthermore, the antibacterial properties of GO (from $85 \mu \mathrm{g} / \mathrm{mL}$ to $1 \mathrm{mg} / \mathrm{mL}$ ) 
toward strains of two epilithic cyanobacteria, Oculatella subterranea and Scytonema julianum, isolated from Roman catacombs, seemed to inhibit in vitro biofilm growth, and for this reason, it is suggested that GO could be suitable for the restoration of stone artifacts. ${ }^{251}$

Microalgae are highly ecologically relevant as primary producers as they are also at the base of the trophic food chain in aquatic ecosystems. The toxicity of carbon-based nanomaterials in algae is mainly due to interactions with the cell surface but is also due to other factors, including shading (reducing their photosynthetic activity), oxidative stress, or sequestration of nutrients. ${ }^{252}$ Exposure of Scenedesmus obliquus to rGO for $72 \mathrm{~h}$ suppressed growth and inhibited chlorophyll a and chlorophyll b levels, apparently due to increased oxidative stress. ${ }^{253}$ rGO significantly downregulated photosystem II activity due to the coating of the rGO on the algal cell surface. GO, rGO, and multilayer graphene (MLG) exhibited much higher toxicity than other carbonacous materials (i.e., carbon nanotubes and graphite) to Chlorella pyrenoidosa. $^{254}$ The shading effect was incriminated in the growth inhibition by GO due to its higher dispersibility and transformation, whereas the other GBMs did not show such effects. It can be questioned whether the decrease of light reaching the photosystems due to the formation of algal-nanocarbon aggregates $^{255-257}$ is sufficient evidence of GBM toxicity, as microalgae can typically optimize their photosystems to the light environment through photoacclimation. ${ }^{258}$ Therefore, some variations in the growth rates reported in the literature might be the result of observation times incompatible with photoacclimation phenomena. In a recent publication from the Graphene Flagship using the aquatic benthic diatom Nitzschia palea, it has been shown that MLG is able to induce growth inhibition only in the first hours of contamination. ${ }^{259}$ These results could be explained by direct contact with the diatoms and to the shading effect. However, the extracellular polymeric substances (EPS) mainly composed of polysaccharides and proteins naturally secreted by diatoms - showed a strong interaction with graphene, leading to growth recovery after trapping of the EPS. ${ }^{259}$ The latter study implies that the presence of an "eco-corona" may impact on the ecotoxicity of GBMs in analogy with the presence of a biocorona in the human body.

More recently, aeroterrestrial green microalgae (AGMs) were also studied in relation to GBMs. AGMs are a small group of polyphyletic origin, with a relatively low substrate specificity and with a strong tendency to be cosmopolitan. ${ }^{260}$ AGMs are able to survive high UV radiation, temperature extremes, and prolonged periods without liquid water in the desiccated state (desiccationtolerant species), ${ }^{261}$ and some enter into symbiotic relationship with fungi (lichenization). These species were not negatively affected by short ( 30 and $60 \mathrm{~min}$ ) and long ( 4 weeks) exposures to FLG and GO. Potential oxidative effects of the same GBMs were also studied through the analysis of quantum yield of primary photochemistry in the dark-adapted state and changes of gene expression of eight genes encoding antioxidant enzymes and stress-related proteins in the lichen photobiont Trebouxia gelatinosa. $^{262}$ Interestingly, GO was found to be inert, and FLG caused the downregulation of a single gene (HSP70), although this did not correspond to a decrease in the expression of HSP70 protein. These studies suggest a negligible effect of GBMs on AGMs which likely can withstand the interaction with these materials thanks to their constitutive adaptation to extreme environments and the avoidance of internalization of GBMs as a result (in Trebouxia) of a thick cell wall.

Effects of Graphene-Based Materials on Seed Plants. As primary producers, seed plants are essential base components of all terrestrial ecosystems. Under the assumption that aerodispersed GBMs will eventually settle over the vegetation as wet or dry depositions and thus will reach the soil, ${ }^{263}$ seed plants are considered as potent media for the transfer of absorbed nanomaterials to the biota through the food chain. For this reason, the effects of GBMs on seed plants have been studied at different growth stages, from seed to seedling, more rarely in the adult plant, but often starting from cell cultures. So far, widely variable effects have been reported, possibly owing to different experimental conditions (i.e., materials, concentrations, exposure time, protocols, etc.) and/or species tested. Using cell suspensions of the model plant, Arabidopsis thaliana exposed to a poorly characterized "graphene" (most probably GO), negative effects in terms of nuclear fragmentation, membrane damage, mitochondrial dysfunction, and ROS production and accumulation were noted, leading to induction of cell death. ${ }^{264}$ Instead, no effects were observed on seed germination or development of seed sprouting. In 2 week old seedlings of $A$. thaliana cultured with GO for 2 further weeks, it was observed that the material accumulated in the root system but not in the leaf cells, implying that the plant copes with GO translocation from root to stem or leaves, although GO was found in all the compartments of the cotyledon cells. ${ }^{265,266}$ Further studies have revealed more problematic effects on seed germination and seedling growth. Hence, although methodological problems cannot be ruled out due to the low number of samples, germination inhibition has been reported for wheat (Triticum aestivum) and broad bean (Vicia faba) when exposed to graphene and GO. In wheat, GO inhibited the germination of seeds at high concentrations and was observed to accumulate in the root, with a limited translocation to stem and leaves, inducing oxidative stress. ${ }^{267}$ In rice seed, delayed germination rates were observed with increasing graphene concentrations $(50 \mu \mathrm{g} / \mathrm{mL}$ and above), and the growth of radicle and plumule was inhibited. ${ }^{268}$ Notably, graphene at a concentration of $5 \mu \mathrm{g} / \mathrm{mL}$ improved some growth indexes. Indeed, carbon-based nanomaterials may have beneficial effects in plants, although the mechanisms remain poorly understood. For example, the absorption of GO by the roots in Vicia faba was found to have both beneficial and toxic effects depending on the concentration. $^{269}$ Increased $V$. faba sensitivity at the highest doses was apparently due to an increased oxidative stress and a concomitant impairment of glutathione metabolism, whereas lower concentrations showed positive effects. In spite of their protective cell walls, harmful effects of carbon-based nanomaterials have also been reported in adult seed plants (reviewed in ref 270). For instance, the leaves of cabbage, spinach, and tomato exhibited a decrease in size after in vivo exposure to GO and a decrease in number due to oxidative-stress-mediated cell death by necrosis. ${ }^{271}$

Aero-dispersed GBMs could interfere with a particularly delicate phase of seed plant life, i.e., fecundation. This process is fundamental for the reproduction of almost all seed plants, but it is also important for humankind as the yield of crop species, largely consisting in seed and fruits, relies on this very important process. The interaction between GBMs and pollen grains might occur directly in the air (anemophilous pollen) or over the stigmatic surfaces of the flowers (all pollen types). Recent in vitro experiments on pollen performance in the model species Nicotiana tabacum and in the nonmodel Corylus avellana showed that pollen germination and tube elongation were affected at GO concentrations $\geq 50 \mu \mathrm{g} / \mathrm{mL}$, decreasing by 20 and $19 \%$ in $N$. tabacum and by 68 and $58 \%$ in C. avellana, respectively. ${ }^{272}$ The frequency of bended tubes increased in N. tabacum. Ratiometric $\mathrm{pH}$ 
indicator studies revealed that $\mathrm{GO}$ affects intracellular $\mathrm{pH}$ homeostasis. Further experiments on C. avellana demonstrated that the main factor influencing pollen performances is the acidic property of GO. FLG also showed a minimal negative effect on pollen tube elongation, probably due to physical interactions with the pectin-rich wall of the pollen tube and/or $\mathrm{Ca}^{2+}$ sequestration, whereas pollen germination and pollen tube growth were not affected by rGO. ${ }^{272}$

Effects of Graphene-Based Materials on Invertebrates. Aquatic and terrestrial invertebrates are likely to be exposed to carbon-based nanomaterials as they accumulate in the terrestrial/ sedimentary compartment. For terrestrial effects, most studies are carried out with worms, especially the nematode Caenorhabditis elegans, a model system that is amenable to mechanistic studies. Zhang et al. studied nanosized GO and GO modified with PEGylated poly-L-lysine using C. elegans and proposed a mechanism of toxicity under stress conditions involving the overproduction of hydroxyl radicals and the formation of oxidizing cytochrome $c$ intermediates. ${ }^{273}$ Furthermore, in a high-throughput study encompassing 20 different nanomaterials, GO was found to be the most toxic toward C. elegans among the carbonbased nanomaterials, followed by rGO and graphene. ${ }^{274}$ Zhao et al. reported that nanosized GO triggered reproductive toxicity with germ cell apoptosis. ${ }^{275}$ Notably, the authors identified an epigenetic, miRNA-based regulatory mechanism activated by GO to suppress the induced reproductive toxicity. The same authors suggested that mir-231 may provide a protective mechanism against toxicity of GO by suppressing the function of the SMK-1-DAF-16 signaling in nematodes. ${ }^{276}$ Ren et al. showed activation of a series of antimicrobial proteins in the nematode after exposure to GO. ${ }^{277}$ In contrast, graphite nanoplatelets did not affect longevity and reproductive capability in C. elegans. ${ }^{278}$ The authors deployed FTIR for mapping the spatial distribution of this material in nematodes. In the insect, Acheta domesticus, commonly known as the house cricket, oxidative stress was observed after injection of pure and manganesecontaminated GO into the hemolymph, a tissue/fluid similar to blood in vertebrates. ${ }^{279}$

In the aquatic environment, pelagic species living in the water column and benthic species living near or within the sediment could be naturally impacted by the presence of carbon-based nanomaterials, depending on their bioavailability to pelagic/ benthic organisms. There are few studies on the response of invertebrates to GBMs, especially with benthic habitat. Artemia salina exposed to GO exhibited no acute toxicity even when GO aggregated in the intestine. ${ }^{280}$ Daphnia magna exhibited an accumulation of graphene on the order of $1 \%$ of the body's dry mass after exposure to $250 \mu \mathrm{g} / \mathrm{L}$ of ${ }^{14} \mathrm{C}$-labeled graphene for $24 \mathrm{~h}$; accumulated graphene in adult Daphnia was likely transferred to neonates. ${ }^{281}$ In the cladoceran, Ceriodaphnia dubia, a significant decrease in the number of neonates and in feeding rates was observed after exposure to GO. ${ }^{282}$ GO impacted the regenerative capacity of the polychaete, Diopatra neapolitana exposed to higher concentrations regenerating less segments and taking longer periods to completely regenerate and altered energy-related responses, especially glycogen content. ${ }^{283}$ In the oligochaete, Tubifex tubifex, no mortality was observed following GO exposure, whereas burrowing activity was significantly reduced. ${ }^{284}$ The toxicity of GO toward the protozoa Euglena gracilis was evidenced by the inhibition of growth and the enhancement of malondialdehyde content and antioxidant enzyme activities. ${ }^{285}$ Some benthic species may have pelagic developmental stages, such as the marine crustacean Amphibalanus amphitrite, whose larvae showed mobility inhibition, as well as mortality, after exposure to GO. ${ }^{286}$

One important factor that can influence the behavior of nanomaterials in the environment is the presence of natural organic material, ubiquitous in natural aquatic environmentsits main components being humic substances (approximately $50 \%$ ), polysaccharides, lipids, proteins, and other organic materials. ${ }^{287}$ Castro et al. recently evaluated the effect of GO on aquatic ecosystems considering the interaction with humic acid on nine different organisms: Raphidocelis subcapitata (green algae), Lemna minor (aquatic plant), Lactuca sativa (lettuce), Daphnia magna (planktonic microcrustacea), Artemia salina (brine shrimp), Chironomus sancticaroli (chironomidae), Hydra attenuata (freshwater polyp), and C. elegans and Panagrolaimus sp. (nematodes). ${ }^{288}$ Overall, GO showed low acute toxicity for the aquatic bioindicator organisms included in the study. Interestingly, the presence of humic acid in the medium increased its colloidal stability in some cases and caused an increase in the toxicity of GO to microcrustaceans (growth rate) and to C. elegans (fertility and reproduction). ${ }^{288}$ The authors proposed that the approach could be useful for predicting ecologically safe GO concentrations and that it could also support environmental risk assessment of GBMs.

Effects of Graphene-Based Materials on Vertebrates. The most studied vertebrates in ecotoxicology are aquatic juvenile fishes and amphibian larvae. Among fishes, the zebrafish (Danio rerio) model is well-represented. As pelagic vertebrates, they may show resistance to carbon-based nanomaterials from the embryonic stage despite the widespread biodistribution observed within the body. ${ }^{289}$ In embryos, GO can be integrated into the chorion causing hypoxia and a significant delay in hatching. ${ }^{290}$ A slight inhibition of cell growth (without significant induction of apoptosis) and a slight hatching delay after exposure to GO were also observed. ${ }^{291}$ The latter study suggests that GO is less toxic to aquatic organisms than MWCNTs as the nanotubes yielded a strong growth inhibition at the same concentrations in zebrafish. In adult zebrafish, GO exposure caused an increase in the number of apoptotic and necrotic gill cells, but genotoxicity was not observed. ${ }^{292}$ Zhang et al. reported that the development of zebrafish embryos exposed to "trace concentrations" $(1-100 \mu \mathrm{g} / \mathrm{L})$ of single-layer GO was impaired because of DNA modification, protein carbonylation, and excessive ROS generation. ${ }^{293}$ The authors noted skeletal and cardiac malformations and transcriptomics analyses revealed dysregulation of collagen and matrix metalloproteinase-related genes following exposure to $100 \mu \mathrm{g} / \mathrm{L}$ of GO.

Studies conducted on amphibian larvae (Ambystoma mexicanum) have shown that no mortality or growth inhibition nor any genotoxicity could be observed, despite a high intake of carbonbased nanomaterials in the digestive tract. ${ }^{294}$ In contrast, in Xenopus laevis larvae, the highest concentration of carbon nanotubes resulted in an inhibition of larval growth, which would be related to the presence of agglomerates in the digestive tract. ${ }^{295,296}$ In a recent study conducted in the Graphene Flagship, MLG composed of 2-20 layers was found to be largely nontoxic for Xenopus larva, with growth inhibition only at concentrations of 10 or $50 \mu \mathrm{g} / \mathrm{mL}$ and no signs of genotoxicity or lethality. ${ }^{297}$ Other recent studies in the Graphene Flagship have shown that the effects of FLG, nanodiamonds, carbon nanotubes, oxidized carbon nanotubes, and GO on the growth inhibition in Xenopus larvae are governed by surface area, whereas mass concentration is a poor descriptor of toxicity for these different types of carbon allotropes $^{298,299}$ (Figure 8). Notably, whatever the amphibian 

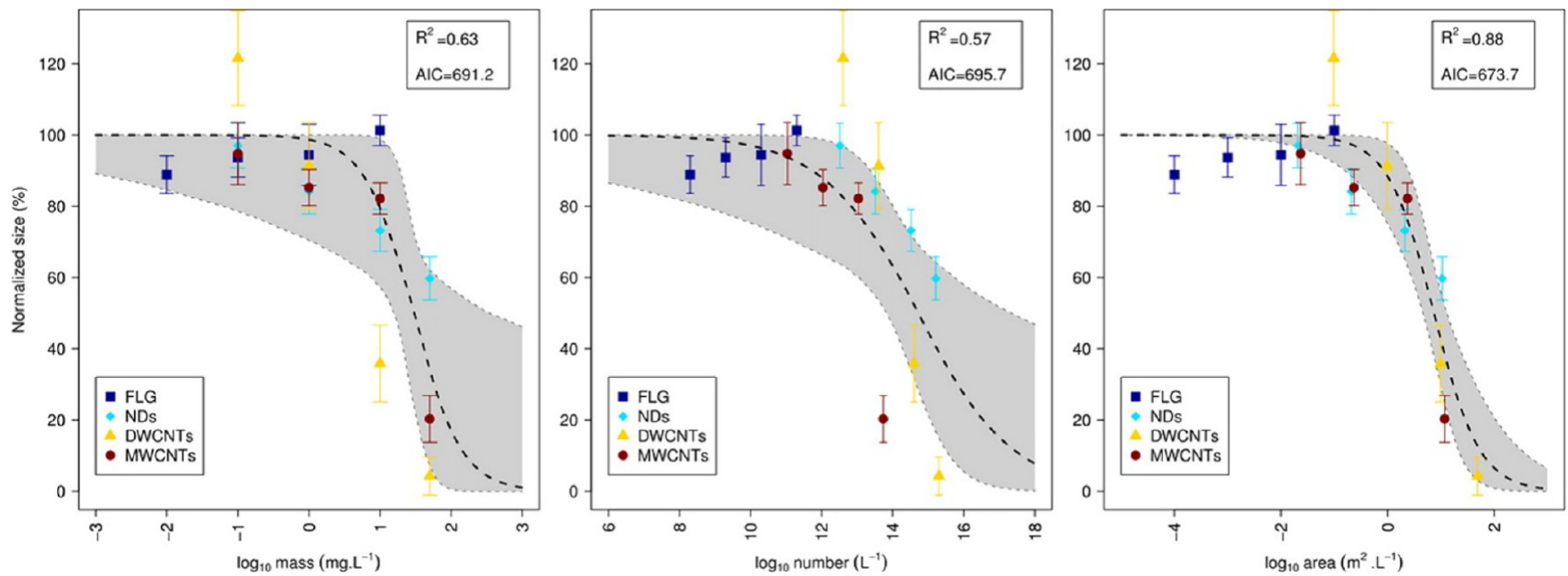

Figure 8. Choosing the best dose metric. Growth inhibition in Xenopus laevis larvae after a 12 day exposure to few layer graphene, nanodiamonds, and double-walled or multiwalled carbon nanotubes. Normalized size (\%) is plotted versus the base-10 logarithms of three different metrics: mass concentration $\left(\mathrm{mg} \cdot \mathrm{L}^{-1}\right)$, number concentration $\left(\mathrm{L}^{-1}\right)$, and surface area concentration $\left(\mathrm{m}^{2} \cdot \mathrm{L}^{-1}\right) . B \mathrm{Black}$ dashed lines represent nonlinear regression model predictions, and shaded areas are $95 \%$ confidence intervals (CIs). The $95 \%$ CIs on the mean sizes are represented as vertical error bars. Reprinted from ref 298. Copyright 2016 American Chemical Society.

organisms, intestinal absorption of carbon-based nanomaterials seems to be limited after oral administration and the materials are then rapidly excreted. ${ }^{295,296}$ The available data suggest that growth inhibition observed in amphibians is related to physical blockage of the gills and/or digestive tract, limiting the exchange surfaces between the gills and/or gut lumen and the internal wall, leading to a decrease in absorption of nutrients and/or gas (anoxia).

Further Research Topics in Ecotoxicology of GrapheneBased Materials. Conventional ecotoxicological approaches using single species are very informative and are needed to evaluate toxicity at the organism level to understand the potential toxicity of GBMs. However, more sophisticated systems are required to get closer to their actual environmental risk assessment. In particular, the notions of biotransformation, bioaccumulation, and biomagnification are generally ignored though they are extremely relevant. Therefore, complex exposure systems with which to evaluate the impact of nanomaterials, particularly through the reconstitution of experimental trophic chains using micro- or mesocosms as experimental tools, are gaining traction. ${ }^{300-302}$ Such systems provide experimental conditions closer to those found in natural ecosystems, but they allow only limited control of biotic and abiotic parameters. These complex systems involving interspecies interactions (e.g., predation and competition) have been used to evaluate the effect of various nanomaterials. ${ }^{303,304}$

Furthermore, another relevant ecotoxicological aspect that is relatively poorly investigated is the impact of "indirect" nanotoxicity, i.e., the toxic amplification of other toxicants or pollutants by nanomaterials. It is fundamental to understand how nanomaterials in general, and GBMs, in particular, interact with other pollutants co-occurring in the environment in terms of adsorption, transport, bioavailability, and the subsequent effects upon pollutant toxicity and biodegradability. For instance, GO can apparently amplify phytotoxicity of arsenic in wheat, Triticum aestivum, ${ }^{305}$ and of cadmium in the freshwater cyanobacterium, Microcystis aeruginosa. ${ }^{250}$ The first conclusion is that GBMs in the environment might lead to a potential enhancement of background contaminants toxicity, even at low nontoxic concentrations. The main limitation of this research field is the enormous number and possible combinations of substances that might deserve to be tested. One additional aspect concerns the assessment of degradation of GBMs released into the environment. There are only a few studies on the capacity of primary decomposers (i.e., bacteria and fungi) to degrade graphitic materials, and the information available so far concerns the effects on the activity of single bacteria ${ }^{306}$ or whole soil bacteria communities. ${ }^{307,308}$ The huge diversity and versatility of bacteria make them the best candidates among all living organisms to study the degradation of carbon-based nanomaterials including GBMs. Their metabolic versatility allows them to use organic materials dispersed in the environment as sources of reduced carbon thanks to extracellular degradation processes. ${ }^{309}$ Furthermore, microbial communities are known to colonize contaminated sites and have the ability to metabolize recalcitrant organic xenobiotics. ${ }^{310}$ White rot fungi may represent an alternative promising field of study because they are able to extrude digestive or oxidative enzymes to break down lignin and other complex organic molecules, and for this reason, they are frequently used in remediation applications. ${ }^{311}$ Previous studies showed that two white rot basidiomycete fungi (Phlebia tremellosa and Trametes versicolor) could oxidize $\mathrm{C}_{60}$ fullerol to $\mathrm{CO}_{2}{ }^{312}$ However, a recent study in which Phanerochaete chrysosporium was exposed for 14 days to GO $(0-4 \mathrm{mg} / \mathrm{mL})$ showed that GO stimulated growth at low concentrations, whereas inhibitory effects were seen at the highest concentrations, with a complete loss of decomposition activity due either to growth inhibition and/or defective enzyme excretion. ${ }^{313}$ On the other hand, rGO was reported to show low toxicity for $P$. chrysosporium. ${ }^{314}$ Overall, there are relatively few studies concerning biodegradation of GBMs in the natural environment, and the environmental fate of GBMs is still largely unknown. In addition, we believe that further studies are needed to understand whether GBMs may elicit toxicity amplification of other environmental pollutants. However, the data on GBMs provided by using numerous vertebrate and nonvertebrate organisms could be used to inform in silico toxicity models and the development of adverse outcome pathways (AOPs). The AOP concept, first presented as a conceptual framework to support ecotoxicology research and risk assessment, ${ }^{315}$ has attracted a great deal of attention in recent years. The overall objective is to support regulatory decision 
making, such as hazard identification and risk assessment, by delineating the key events leading to adverse outcomes. ${ }^{316}$ AOPs are chemically "agnostic" in the sense that they describe in a generalized way how a molecular initiating event is linked to an adverse outcome via so-called key events. Nonethelesss, the rich source of hazard data emerging for GBMs could be profitably exploited in the framework of environmental risk assessment and help in the understanding of the contribution of GBMs in the adverse effects observed in humans and wildlife at larger organizational scale.

\section{EXPOSURE AND LIFE CYCLE ANALYSIS OF GRAPHENE-BASED MATERIALS}

The main human exposure of concern at present is pulmonary exposure in workers during the production and handling of GBMs, even though dermal or oral exposure may also occur. ${ }^{4}$ Naturally, the safety of scientists and students producing or studying nanomaterials including GBMs should not be neglected, though the release of nanomaterials in such workplaces is likely to be very low according to recent studies. ${ }^{317,318}$ Future biomedical applications of GBMs will also lead to exposure in patients, but all (novel) medicines and medical devices need to be evaluated for safety.

Occupational Exposure to Graphene-Based Materials. Five studies related to occupational exposure of GBMs are available. The GBMs were produced by using a graphite exfoliation, ${ }^{319,320}$ a chemical vapor deposition (CVD) process, ${ }^{166,320}$ and through a nonspecified technique, most likely CVD. ${ }^{321,322}$ Unfortunately, these studies did not report the GBM specifications or production volumes. Thus, some results can be applied for GBMs at laboratory scale production volumes (less than grams $)^{166,320}$ and some at industrial scale production. ${ }^{319,321,322}$ Spinazzè et al. performed long-term measurements, ${ }^{319}$ whereas the other studies were performed over one process cycle. Thus, the variation in exposure levels is not well-known. However, all the studies in this sample showed that exposure levels were very low if emission controls were properly applied and good working practices were followed. The National Institute for Occupational Safety and Health in the United States published a report on engineering controls for production and handling of GBMs (i.e., GNPs). ${ }^{321}$ The authors measured worker breathing zone concentration levels during production of GBMs using two different similar processes for large batches (P1) and small batches (P2). They reported the concentrations measured during product harvesting and process tank cleaning. Product harvesting was made without using exposure controls at $\mathrm{P} 1$ or by using a blower located downstream and butterfly valves incorporated on the upstream of the collection vessels at P2. GBM release was detected during product harvesting where a collection container was removed from discharger and during process tank cleaning. Good working practices and proper use of emission controls reduced the exposure levels from 88 to $>99.9 \%$. Bengtson et al. measured graphene exposure levels during synthesis of graphene by a commercially available CVD system without using any engineered emission controls. ${ }^{166}$ The measurements were carried out in a clean room and at an industrial site over one production cycle. In the clean room, the background level of particle number concentration range was $<5 \mathrm{~cm}^{-3}$, and the concentration measured next to the hatch by a condensation particle counter remained mainly at the background level. Only reactor opening and dry wiping the reactor increased the concentration up to around $15 \mathrm{~cm}^{-3}$ for a few seconds. Samples contained no particles according to TEM analysis. The authors also could not exclude that the concentration increases during opening of the reactor and dry wiping were due to disturbance of tubing and flows to the condensation particle counter. In the industrial site, they could not detect an increase in concentrations measured next to the hatch due to high background particle concentration. ${ }^{166}$ Lee et al. studied worker exposure during laboratoryscale production of graphene using a graphite exfoliation and CVD processes and transferring the graphene to a polyethylene terephthalate (PET) sheet. ${ }^{320}$ The graphite exfoliation is made in liquid, which is not expected to release airborne particles. The highest release potential was expected to be during sonication, but the process was enclosed and GBMs were not detected in the air. During the CVD process, particle number concentration increased for a short period during GBM collection. The transfer of the GBMs to the PET sheets and the cutting of the sheets did not result in any detectable increase in concentrations. GBMlike structures were found from particle samples collected from air of both production areas. Spinazzè et al. performed six measurement campaigns during a period of 12 months in an industrial facility with a GBM production capacity of 30 tons per year. ${ }^{319}$ The manufacturing process consisted of the following steps: (1) acceptance of raw materials (graphite) and storage, (2) plasma expansion, (3) postplasma treatment/exfoliation performed in liquid media, (4) drying, (5) finishing operations (e.g., packaging), and (6) storage of final products. The process was automated and the workers' tasks were acquiring samples for quality control, cleaning, and maintenance operations. The estimated $8 \mathrm{~h}$ time-weighted average concentrations ranged from 909 to 6438 particles $/ \mathrm{cm}^{3}$ and from 0.38 to $3.86 \mu \mathrm{g} / \mathrm{m}^{3}$. Gravimetric analysis of cascade impactor samples from the graphene expansion room showed that $65 \%$ of the mass was in the size range of $250-500 \mathrm{~nm}$. However, the authors did not analyze the composition of the airborne particles. Nonetheless, the study suggested, overall, that significant exposure of workers to GBMs is unlikely. However, the results also indicated that workers who are directly involved in specific tasks (e.g., material sampling for quality control) have a higher potential for occupational exposure than those involved in routine production. ${ }^{319}$

Life Cycle Analysis Approaches for Graphene-Based Materials. Information on toxicity, biodistribution, fate, and exposure are essential to understand the hazard of introducing GBMs in the environment, but they can also be useful to assess the environmental sustainability of producing and using GBMs. The life cycle assessment (LCA) methodology provides a framework for such an assessment by combining models of fabrication processes and their associated supply chains with models of GBM interactions in the environment. ${ }^{323}$ Here, we overview recent LCA studies on GBMs to highlight the current state of knowledge as well as gaps in the environmental sustainability assessments. Thus far, LCA studies have focused on identifying the main sources of environmental impacts for graphene, ${ }^{324,325}$ graphite nanoplatelets, ${ }^{326}$ and reduced GO. ${ }^{32}$ These studies have addressed different fabrication methods such as chemical reduction, ultrasonication exfoliation, thermal exfoliation combined with ball-milling, chemical vapor deposition, and epitaxial growth. For instance, studies performed in the frame of the MISTRA Environmental Nanosafety project have highlighted differences for graphene produced by ultrasonication or chemical reduction in terms of energy and water use as well as human and ecotoxicity ${ }^{327}$ (Figure 9). The results of these studies vary significantly because of the range of considered GBMs, fabrication methods, and scales of production. However, they all show that energy consumption and chemicals used (e.g., diethyl ether and methane) are the two most important sources 

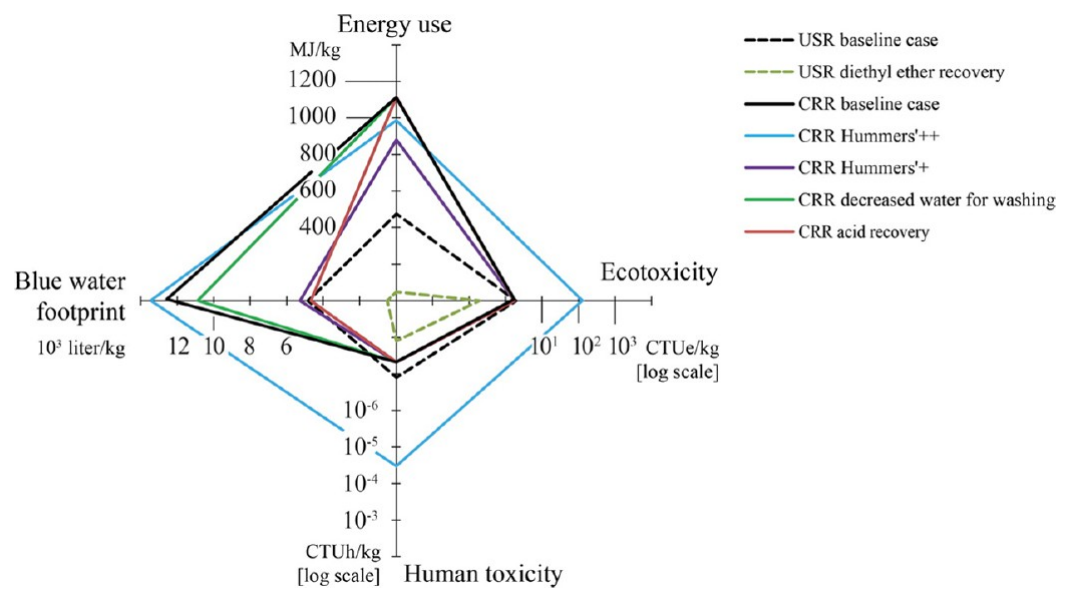

[log scale] Human toxicity

Figure 9. Life cycle analysis of graphene production. The chemical reduction route (CRR) and the ultrasonication route (USR) are two liquid phase exfoliation routes with industrial-scale potential at low costs. The figure illustrates the results of the sensitivity analysis. Reprinted from ref 218. Copyright 2016 American Chemical Society.

of environmental impacts for categories such as global warming potential, freshwater ecotoxicity, human toxicity, and water use. Although these recent LCA studies offer some insights into the environmental sustainability of GBMs, key issues remain to be addressed. The main concern originates from the sources of data for these studies as they are based on scientific papers, patents, and prospective models which instigate model uncertainties that are still difficult to evaluate. Additionally, other assumptions on the fabrication methods could be refined. For example, Arvidsson et al. used a 95-99\% yield hypothesis for the ultrasonication process of graphite into graphene, ${ }^{327}$ but such a high yield would probably raise concerns on the purity of the fabricated graphene. This example thus raises the issue of providing an appropriate environmental sustainability assessment for a wide range of GBMs as a function of their properties that can vary significantly and affect their usefulness in different applications. In this context, the properties that should be used for classifying the different GBMs must be related to the applications of the materials. Thus, properties such as mechanical strength, electrical conductivity, thermal conductivity, optical absorption, and surface-tomass ratio could all be relevant to define such groups. The definition of such groups would allow for relevant comparisons with respect to the environmental impact. However, it remains important to consider prospective scenarios for these fabrication models as it is expected that applications of graphene will not reach maturity before the period 2025-2030, ${ }^{1}$ and the electricity mixes worldwide-key inputs when it comes to energy consumption-are expected to evolve significantly in their composition (and thus in their related environmental impacts) if greenhouse gas targets are to be met at the international level.

The other important concern that arises from the recent LCA studies on GBMs is the lack of consideration for the human and ecotoxicity of GBMs. This omission is currently explained by the lack of GBM specific characterization factors (CFs) within the existing life cycle impact assessment framework. Such CFs would, in theory, translate the effects of GBM emissions into potential toxicity effects on humans, animals, plants, and other living organisms of the environment. The so-called USETox method developed under the auspices of the UNEP/SETAC Life Cycle Initiative ${ }^{328}$ is currently the recommended method for providing such CFs within the LCA framework, but it requires toxicity results from in vivo studies at different trophic levels and it is not specifically designed for nanomaterials. The method also requires models for the fate and exposition to nanomaterials. Some progress has been made in this regard by Salieri et al..$^{329}$ and Ettrup et al. ${ }^{330}$ Both groups have used and modified the so-called Simplebox4nano multimedia model ${ }^{331}$ and calculated aquatic toxicity CFs for $\mathrm{TiO}_{2}$ nanoparticles. Overall, these specific fate and exposure models could be modified to be used for other nanomaterial such as GBMs (reviewed in ref 332). However, only preliminary evaluations of toxicity CFs for GBMs can be extrapolated from the available data. Therefore, it is not expected that LCA studies will be able to offer a complete evaluation of potential impacts of GBMs until further in vivo toxicity studies are carried out.

\section{CONCLUDING REMARKS}

With the present review, we have attempted to give an overview of the state-of-the-art of human and environmental hazard assessment of GBMs and to highlight the importance of understanding the structure-activity relationships that underlie the potential toxicity of these materials. For this to happen, we need to "know the materials". ${ }^{333}$ In addition, it is equally important to use robust and validated assays for toxicological testing with respect to human health and environmental safety. ${ }^{334}$ Furthermore, whereas research on GBMs should address issues relevant for risk assessment, ${ }^{4}$ studies are also needed that address the fundamental aspects of their biological interactions. ${ }^{335,336}$ To this end, systems biology approaches provide a means with which to dissect the mechanisms underlying the adverse effects of GBMs while yielding additional insights into the behavior of this class of biomaterials in living systems. ${ }^{337}$

The present overview of the literature has shown that while the hazard assessment of GBMs is coming of age, with ever increasing numbers of studies addressing the potential impact of GBMs on living systems, data gaps still remain, and this, therefore, precludes the prediction of toxicity based solely on material properties of GBMs. Indeed, we have shown that for some selected end-points, one may begin to see a (predictable) pattern of effects (refer to supporting Figures S1-S5 and Tables $\mathrm{S} 1-\mathrm{S} 5)$, but it is also clear that the chemical space of graphene and its derivatives is yet to be fully explored (Figure 1). However, it is hoped that as this framework is populated with additional studies, ideally using libraries of GBMs, or at any rate using GBMs that have undergone rigorous characterization, the structure-activity relationships of these materials may reveal 
themselves. Indeed, it is important to move from a descriptive to a predictive toxicology. $\mathrm{Nel}$ and co-workers ${ }^{338}$ proposed the use of mechanism-based high-throughput screening to make predictions about the physicochemical properties of nanomaterials that may lead to disease outcomes in living organisms. Integral to this approach is the fact that the majority of screening assays are carried out in vitro while critical validation assays are performed in animals or in whole organisms, e.g., zebrafish embryos. To this, one may add that systems toxicology approaches also may shed light on the interactions of nanomaterials with living organisms. ${ }^{339}$ Moreover, omics data sets (e.g., transcriptomics, proteomics, and metabolomics) can aid in the identification of molecular initiating events and provide supportive evidence of key events at different levels of biological organization, thus enriching AOPs. ${ }^{340}$ AOPs, in turn, could aid in the development of predictive models, ultimately supporting risk assessment of chemicals and nanomaterials ${ }^{341}$ including GBMs. The European Commission's Joint Research Centre (JRC) recently published a review of the current status of computational methods that are potentially useful for predicting the properties of engineered nanomaterials. ${ }^{342}$ The authors identified several issues hampering the development, uptake, and use of such models, including methods for physicochemical and hazard characterization, sharing and accessibility of data, regulatory applicability of the models, and so on. Indeed, to fully exploit (quantitative) structure-activity relationship or (Q)SAR modeling, stronger collaborations between experimental scientists and modelers are required. ${ }^{343}$ Nonetheless, in silico approaches could provide a means of extracting non-obvious structure-activity relationships of GBMs provided that the test materials are wellcharacterized and test systems are robust. In 2017, the European Commission published eight NanoData Landscape Compilation reports (see https://publications.europa.eu/en/). These reports offer a snapshot of the environment for nanotechnology in different application fields. In the report on "health", GBMs are hardly mentioned, whereas in the report on "environment", the authors have stated that "based on the scarce available evidence, it cannot be excluded that some forms of graphene will be as potent a toxicant as carbon nanotubes". This statement raises the spectre of asbestos-like properties of carbon nanotubes, ${ }^{5}$ but according to a recent report published by the International Agency for Research on Cancer (IARC), only certain types of rigid, multiwalled carbon nanotubes can be classified as being possibly carcinogenic to humans. ${ }^{344}$ Moreover, as we have discussed at length in the present review, GBMs cannot be grouped together as one material. Indeed, GBMs differ with respect to three key parameters: the number of graphene layers, average lateral dimensions, and carbon-to-oxygen atomic ratio. ${ }^{22}$ Furthermore, GBMs can be functionalized in a multitude of different ways, thereby changing their properties and, in all likelihood, their biological behavior. The fact that $\mathrm{GO}^{140}$ and $\mathrm{FLG}^{345}$ can be digested by cells of the immune system implies that these materials are not necessarily biopersistent. Notably, research conducted in the context of the Graphene Flagship and by other investigators in the past several years has shown that the hazard potential for different members of the GBM family may vary considerably, and it is not a valid statement that all GBMs are as hazardous as carbon nanotubes, nor is it true that all carbon nanotubes are hazardous. In fact, the devil is in the details, and careful characterization of material properties is of critical importance. Furthermore, it is equally important that the material properties are reported in full in papers dealing with (eco)toxicity assessment of GBMs. Can the information that has been collected on safety of GBMs be applied to other 2D materials? We believe that some aspects might be common to all $2 \mathrm{D}$ materials, or even to all nanomaterials, while some "postcarbon" 2D materials will likely present with their own specific concerns. For instance, the propensity to dissolve in a biological environment with the release of ionic species that are more biologically/chemically reactive than the parental $2 \mathrm{D}$ material is an issue that has not been described for GBMs. ${ }^{346}$ Moreover, Guiney et al. ${ }^{347}$ recently commented that "with a constantly expanding library of $2 \mathrm{D}$ materials, the ability to predict toxicological outcomes is of critical importance" and suggested that high-throughput screening approaches may prove useful in order to elucidate cellular interactions of $2 \mathrm{D}$ materials. However, the issue is not so much the low throughput of current approaches as much as the inconsistent design of commonly used toxicity assays and frequent lack of material characterization. Indeed, careful characterization of both the test material and the test system is required, and a proposal was recently put forward for minimum reporting requirements in publications dealing with nanobiointeractions. ${ }^{348}$ Though such reporting requirements have not yet been adopted, it is important to discuss these issues in the scientific community. To conclude, the hype that inevitably follows with technological advances should be tempered by sound, science-based assessment of the potential impact on human health and the environment to ensure safe and sustainable development of new products and applications. The present survey of the literature can perhaps serve as a first step toward a systematic collection of data on the safety or biocompatibility of GBMs.

\section{ASSOCIATED CONTENT}

\section{S Supporting Information}

The Supporting Information is available free of charge on the ACS Publications website at DOI: 10.1021/acsnano.8b04758. Five figures and five tables describing $3 \mathrm{D}$ plots to illustrate the range of GBMs that have been subjected to toxicological studies (PDF)

\section{AUTHOR INFORMATION}

\section{Corresponding Authors}

*E-mail: bengt.fadeel@ki.se.

*E-mail: prato@units.it.

*E-mail: a.bianco@ibmc-cnrs.unistra.fr.

ORCID *

Bengt Fadeel: 0000-0001-5559-8482

Cyrill Bussy: 0000-0001-8870-443X

Sonia Merino: 0000-0002-7124-8076

Ester Vázquez: 0000-0003-3223-8024

Emmanuel Flahaut: 0000-0001-8344-6902

Florence Mouchet: 0000-0003-1993-8448

Lauris Evariste: 0000-0001-8718-7776

Antti J. Koivisto: 0000-0002-6769-1999

Ulla Vogel: 0000-0001-6807-1524

Cristina Martín: 0000-0001-5670-3328

Lucia G. Delogu: 0000-0002-2329-7260

Tina Buerki-Thurnherr: 0000-0003-3723-6562

Peter Wick: 0000-0002-0079-4344

Didier Beloin-Saint-Pierre: 0000-0003-1214-8195

Roland Hischier: 0000-0002-1084-7665

Marco Pelin: 0000-0002-4306-7411

Fabio Candotto Carniel: 0000-0002-8277-4725

Mauro Tretiach: 0000-0002-0529-1359 
Fabrizia Cesca: 0000-0003-2190-6314

Fabio Benfenati: 0000-0002-0653-8368

Denis Scaini: 0000-0001-8398-8074

Laura Ballerini: 0000-0001-8420-0787

Kostas Kostarelos: 0000-0002-2224-6672

Maurizio Prato: 0000-0002-8869-8612

Alberto Bianco: 0000-0002-1090-296X

Notes

The authors declare no competing financial interest.

\section{ACKNOWLEDGMENTS}

The authors are supported by the European Commission through the Graphene Flagship Project (Grant Agreement No. 696656), the JTC 2015 FLAG-ERA project (G-Immunomics), and the FP7-GLADIATOR project (Grant Agreement No. 604000). B.F. is also supported, in part, by the national MISTRA Environmental Nanosafety Program (Sweden). A.B. wishes to thank the French National Research Agency (ANR-15-GRFL0001-05) and the International Center for Frontier Research in Chemistry (icFRC) for funding.

\section{VOCABULARY}

graphene-based materials, family of carbon-based materials including graphene, graphene oxide, reduced graphene oxide, and graphene quantum dots; life cycle assessment, technique for examining the inputs and outputs of materials and the associated environmental impacts directly attributable to a product throughout its life cycle; systems biology, approach in biomedical research to understand the complexity at organism, tissue or cellular level, leading to a deeper comprehension of complex biological networks and processes; nanosafety, safety issues associated with nanotechnologies, encompassing topics like nanomaterial characterization, effects of nanomaterials on human health and the environment, and exposure and risk assessment; biodegradation, process by which a microorganism transforms or alters the structure of an organic material through metabolic or enzymatic actions; biodistribution, determination of the location of compounds traveling within an organism

\section{REFERENCES}

(1) Novoselov, K. S.; Fal'ko, V. I.; Colombo, L.; Gellert, P. R.; Schwab, M. G.; Kim, K. A Roadmap for Graphene. Nature 2012, 490, 192-200.

(2) Ferrari, A. C.; Bonaccorso, F.; Fal'ko, V.; Novoselov, K. S.; Roche, S.; Bøggild, P.; Borini, S.; Koppens, F. H. L.; Palermo, V.; Pugno, N.; Garrido, J. A.; Sordan, R.; Bianco, A.; Ballerini, L.; Prato, M.; Lidorikis, E.; Kivioja, J.; Marinelli, C.; Ryhänen, T.; Morpurgo, A.; et al. Science and Technology Roadmap for Graphene, Related Two-Dimensional Crystals, and Hybrid Systems. Nanoscale 2015, 7, 4598-4810.

(3) Bianco, A.; Prato, M. Safety Concerns on Graphene and 2D Materials: A Flagship Perspective. 2D Mater. 2015, 2, 030201.

(4) Park, M. V. D. Z.; Bleeker, E. A. J.; Brand, W.; Cassee, F. R.; van Elk, M.; Gosens, I.; de Jong, W. H.; Meesters, J. A. J.; Peijnenburg, W. J. G. M.; Quik, J. T. K.; Vandebriel, R. J.; Sips, A. J. A. M. Considerations for Safe Innovation: The Case of Graphene. ACS Nano 2017, 11, 9574-9593.

(5) Poland, C. A.; Duffin, R.; Kinloch, I.; Maynard, A.; Wallace, W. A. H.; Seaton, A.; Stone, V.; Brown, S.; MacNee, W.; Donaldson, K. Carbon Nanotubes Introduced into the Abdominal Cavity of Mice Show Asbestos-like Pathogenicity in a Pilot Study. Nat. Nanotechnol. 2008, 3, 423-428.

(6) Kuempel, E. D.; Jaurand, M.-C.; Møller, P.; Morimoto, Y.; Kobayashi, N.; Pinkerton, K. E.; Sargent, L. M.; Vermeulen, R. C. H.; Fubini, B.; Kane, A. B. Evaluating the Mechanistic Evidence and Key Data Gaps in Assessing the Potential Carcinogenicity of Carbon
Nanotubes and Nanofibers in Humans. Crit. Rev. Toxicol. 2017, 47, 158.

(7) Kostarelos, K.; Lacerda, L.; Pastorin, G.; Wu, W.; Wieckowski, S.; Luangsivilay, J.; Godefroy, S.; Pantarotto, D.; Briand, J.-P.; Muller, S.; Prato, M.; Bianco, A. Cellular Uptake of Functionalized Carbon Nanotubes Is Independent of Functional Group and Cell Type. Nat. Nanotechnol. 2007, 2, 108-113.

(8) Schipper, M. L.; Nakayama-Ratchford, N.; Davis, C. R.; Kam, N. W. S.; Chu, P.; Liu, Z.; Sun, X.; Dai, H.; Gambhir, S. S. A Pilot Toxicology Study of Single-Walled Carbon Nanotubes in a Small Sample of Mice. Nat. Nanotechnol. 2008, 3, 216-221.

(9) Kagan, V. E.; Konduru, N. V.; Feng, W.; Allen, B. L.; Conroy, J.; Volkov, Y.; Vlasova, I. I.; Belikova, N. A.; Yanamala, N.; Kapralov, A.; Tyurina, Y. Y.; Shi, J.; Kisin, E. R.; Murray, A. R.; Franks, J.; Stolz, D.; Gou, P.; Klein-Seetharaman, J.; Fadeel, B.; Star, A.; et al. Carbon Nanotubes Degraded by Neutrophil Myeloperoxidase Induce Less Pulmonary Inflammation. Nat. Nanotechnol. 2010, 5, 354-359.

(10) Bhattacharya, K.; Mukherjee, S. P.; Gallud, A.; Burkert, S. C.; Bistarelli, S.; Bellucci, S.; Bottini, M.; Star, A.; Fadeel, B. Biological Interactions of Carbon-Based Nanomaterials: From Coronation to Degradation. Nanomedicine 2016, 12, 333-351.

(11) Shvedova, A. A.; Kagan, V. E.; Fadeel, B. Close Encounters of the Small Kind: Adverse Effects of Man-Made Materials Interfacing with the Nano-Cosmos of Biological Systems. Annu. Rev. Pharmacol. Toxicol. 2010, 50, 63-88.

(12) Donaldson, K.; Poland, C. A. Nanotoxicity: Challenging the Myth of Nano-Specific Toxicity. Curr. Opin. Biotechnol. 2013, 24, 724734.

(13) Bianco, A. Graphene: Safe or Toxic? The Two Faces of the Medal. Angew. Chem., Int. Ed. 2013, 52, 4986-4997.

(14) Krug, H. F. Nanosafety Research-Are We on the Right Track? Angew. Chem., Int. Ed. 2014, 53, 12304-12319.

(15) Sanchez, V. C.; Jachak, A.; Hurt, R. H.; Kane, A. B. Biological Interactions of Graphene-Family Nanomaterials: An Interdisciplinary Review. Chem. Res. Toxicol. 2012, 25, 15-34.

(16) Lalwani, G.; D’Agati, M.; Khan, A. M.; Sitharaman, B. Toxicology of Graphene-Based Nanomaterials. Adv. Drug Delivery Rev. 2016, 105, 109-144.

(17) Reina, G.; González-Domínguez, J. M.; Criado, A.; Vázquez, E.; Bianco, A.; Prato, M. Promises, Facts and Challenges for Graphene in Biomedical Applications. Chem. Soc. Rev. 2017, 46, 4400-4416.

(18) Ema, M.; Gamo, M.; Honda, K. A Review of Toxicity Studies on Graphene-Based Nanomaterials in Laboratory Animals. Regul. Toxicol. Pharmacol. 2017, 85, 7-24.

(19) Fojtů, M.; Teo, W. Z.; Pumera, M. Environmental Impact and Potential Health Risks of 2D Nanomaterials. Environ. Sci.: Nano 2017, 4, 1617-1633.

(20) Volkov, Y.; McIntyre, J.; Prina-Mello, A. Graphene Toxicity as a Double-Edged Sword of Risks and Exploitable Opportunities: A Critical Analysis of the Most Recent Trends and Developments. 2D Mater. 2017, 4, 022001.

(21) Bianco, A.; Cheng, H.-M.; Enoki, T.; Gogotsi, Y.; Hurt, R. H.; Koratkar, N.; Kyotani, T.; Monthioux, M.; Park, C. R.; Tascon, J. M. D.; Zhang, J. All in the Graphene Family - A Recommended Nomenclature for Two-Dimensional Carbon Materials. Carbon 2013, $65,1-6$.

(22) Wick, P.; Louw-Gaume, A. E.; Kucki, M.; Krug, H. F.; Kostarelos, K.; Fadeel, B.; Dawson, K. a.; Salvati, A.; Vázquez, E.; Ballerini, L.; Tretiach, M.; Benfenati, F.; Flahaut, E.; Gauthier, L.; Prato, M.; Bianco, A. Classification Framework for Graphene-Based Materials. Angew. Chem., Int. Ed. 2014, 53, 7714-7718.

(23) Bottari, G.; Herranz, M. Á.; Wibmer, L.; Volland, M.; RodríguezPérez, L.; Guldi, D. M.; Hirsch, A.; Martín, N.; D’Souza, F.; Torres, T. Chemical Functionalization and Characterization of Graphene-Based Materials. Chem. Soc. Rev. 2017, 46, 4464-4500.

(24) Dong, L.; Yang, J.; Chhowalla, M.; Loh, K. P. Synthesis and Reduction of Large Sized Graphene Oxide Sheets. Chem. Soc. Rev. 2017, 46, 7306-7316. 
(25) Yin, P. T.; Shah, S.; Chhowalla, M.; Lee, K.-B. Design, Synthesis, and Characterization of Graphene-Nanoparticle Hybrid Materials for Bioapplications. Chem. Rev. 2015, 115, 2483-2531.

(26) Mukherjee, S. P.; Lozano, N.; Kucki, M.; Del Rio-Castillo, A. E.; Newman, L.; Vázquez, E.; Kostarelos, K.; Wick, P.; Fadeel, B. Detection of Endotoxin Contamination of Graphene Based Materials Using the TNF- $\alpha$ Expression Test and Guidelines for Endotoxin-Free Graphene Oxide Production. PLoS One 2016, 11, e0166816.

(27) Cai, M.; Thorpe, D.; Adamson, D. H.; Schniepp, H. C. Methods of Graphite Exfoliation. J. Mater. Chem. 2012, 22, 24992-25002.

(28) Hernández-Sánchez, D.; Scardamaglia, M.; Saucedo-Anaya, S.; Bittencourt, C.; Quintana, M. Exfoliation of Graphite and Graphite Oxide in Water by Chlorin e 6 . RSC Adv. 2016, 6, 66634-66640.

(29) Salunke, B. K.; Kim, B. S. Facile Synthesis of Graphene Using a Biological Method. RSC Adv. 2016, 6, 17158-17162.

(30) Pattammattel, A.; Pande, P.; Kuttappan, D.; Puglia, M.; Basu, A. K.; Amalaradjou, M. A.; Kumar, C. V. Controlling the Graphene-Bio Interface: Dispersions in Animal Sera for Enhanced Stability and Reduced Toxicity. Langmuir 2017, 33, 14184-14194.

(31) Liu, X.; Liu, J.; Zhan, D.; Yan, J.; Wang, J.; Chao, D.; Lai, L.; Chen, M.; Yin, J.; Shen, Z. Repeated Microwave-Assisted Exfoliation of Expandable Graphite for the Preparation of Large Scale and High Quality Multi-Layer Graphene. RSC Adv. 2013, 3, 11601-11606.

(32) León, V.; González-Domínguez, J. M.; Fierro, J. L. G.; Prato, M.; Vázquez, E. Production and Stability of Mechanochemically Exfoliated Graphene in Water and Culture Media. Nanoscale 2016, 8, 1454814555.

(33) González-Domínguez, J. M.; León, V.; Lucío, M. I.; Prato, M.; Vázquez, E. Production of Ready-to-Use Few-Layer Graphene in Aqueous Suspensions. Nat. Protoc. 2018, 13, 495-506.

(34) Amiri, A.; Zubir, M. N. M.; Dimiev, A. M.; Teng, K. H.; Shanbedi, M.; Kazi, S. N.; Rozali, S. B. Facile, Environmentally Friendly, Cost Effective and Scalable Production of Few-Layered Graphene. Chem. Eng. J. 2017, 326, 1105-1115.

(35) Ali-Boucetta, H.; Bitounis, D.; Raveendran-Nair, R.; Servant, A.; Van den Bossche, J.; Kostarelos, K. Purified Graphene Oxide Dispersions Lack In Vitro Cytotoxicity and In Vivo Pathogenicity. Adv. Healthcare Mater. 2013, 2, 433-441.

(36) Jasim, D. A.; Lozano, N.; Kostarelos, K. Synthesis of FewLayered, High-Purity Graphene Oxide Sheets from Different Graphite Sources for Biology. 2D Mater. 2016, 3, 014006.

(37) Coleman, B. R.; Knight, T.; Gies, V.; Jakubek, Z. J.; Zou, S. Manipulation and Quantification of Graphene Oxide Flake Size: Photoluminescence and Cytotoxicity. ACS Appl. Mater. Interfaces 2017, 9, 28911-28921.

(38) Orecchioni, M.; Jasim, D. A.; Pescatori, M.; Manetti, R.; Fozza, C.; Sgarrella, F.; Bedognetti, D.; Bianco, A.; Kostarelos, K.; Delogu, L. G. Molecular and Genomic Impact of Large and Small Lateral Dimension Graphene Oxide Sheets on Human Immune Cells from Healthy Donors. Adv. Healthcare Mater. 2016, 5, 276-287.

(39) Mukherjee, S. P.; Kostarelos, K.; Fadeel, B. Cytokine Profiling of Primary Human Macrophages Exposed to Endotoxin-Free Graphene Oxide: Size-Independent NLRP3 Inflammasome Activation. Adv. Healthcare Mater. 2018, 7, 1700815.

(40) Jasim, D. A.; Boutin, H.; Fairclough, M.; Ménard-Moyon, C.; Prenant, C.; Bianco, A.; Kostarelos, K. Thickness of Functionalized Graphene Oxide Sheets Plays Critical Role in Tissue Accumulation and Urinary Excretion: A Pilot PET/CT Study. Appl. Mater. Today 2016, 4, 24-30.

(41) Jasim, D. A.; Murphy, S.; Newman, L.; Mironov, A.; Prestat, E.; McCaffrey, J.; Ménard-Moyon, C.; Rodrigues, A. F.; Bianco, A.; Haigh, S.; Lennon, R.; Kostarelos, K. The Effects of Extensive Glomerular Filtration of Thin Graphene Oxide Sheets on Kidney Physiology. ACS Nano 2016, 10, 10753-10767.

(42) Rodrigues, A. F.; Newman, L.; Lozano, N.; Mukherjee, S. P.; Fadeel, B.; Bussy, C.; Kostarelos, K. A Blueprint for the Synthesis and Characterisation of Thin Graphene Oxide with Controlled Lateral Dimensions for Biomedicine. 2D Mater. 2018, 5, 035020.
(43) De Silva, K. K. H.; Huang, H.-H.; Joshi, R. K.; Yoshimura, M. Chemical Reduction of Graphene Oxide Using Green Reductants. Carbon 2017, 119, 190-199.

(44) Fernández-Merino, M. J.; Guardia, L.; Paredes, J. I.; Villar-Rodil, S.; Solís-Fernández, P.; Martínez-Alonso, A.; Tascón, J. M. D. Vitamin $C$ Is an Ideal Substitute for Hydrazine in the Reduction of Graphene Oxide Suspensions. J. Phys. Chem. C 2010, 114, 6426-6432.

(45) Zhu, C.; Guo, S.; Fang, Y.; Dong, S. Reducing Sugar: New Functional Molecules for the Green Synthesis of Graphene Nanosheets. ACS Nano 2010, 4, 2429-2437.

(46) Haghighi, B.; Tabrizi, M. A. Green-Synthesis of Reduced Graphene Oxide Nanosheets Using Rose Water and a Survey on Their Characteristics and Applications. RSC Adv. 2013, 3, 13365-13371.

(47) Akhavan, O.; Ghaderi, E. Escherichia Coli Bacteria Reduce Graphene Oxide to Bactericidal Graphene in a Self-Limiting Manner. Carbon 2012, 50, 1853-1860.

(48) Aunkor, M. T. H.; Mahbubul, I. M.; Saidur, R.; Metselaar, H. S. C. The Green Reduction of Graphene Oxide. RSC Adv. 2016, 6, 27807-27828.

(49) Soltani, T.; Kyu Lee, B. A Benign Ultrasonic Route to Reduced Graphene Oxide from Pristine Graphite. J. Colloid Interface Sci. 2017, 486, 337-343.

(50) Wang, Z.; Xu, D.; Huang, Y.; Wu, Z.; Wang, L.; Zhang, X. Facile, Mild and Fast Thermal-Decomposition Reduction of Graphene Oxide in Air and Its Application in High-Performance Lithium Batteries. Chem. Commun. 2012, 48, 976-978.

(51) Yang, D.; Velamakanni, A.; Bozoklu, G.; Park, S.; Stoller, M.; Piner, R. D.; Stankovich, S.; Jung, I.; Field, D. A.; Ventrice, C. A.; Ruoff, R. S. Chemical Analysis of Graphene Oxide Films after Heat and Chemical Treatments by X-Ray Photoelectron and Micro-Raman Spectroscopy. Carbon 2009, 47, 145-152.

(52) Mori, F.; Kubouchi, M.; Arao, Y. Effect of Graphite Structures on the Productivity and Quality of Few-Layer Graphene in Liquid-Phase Exfoliation. J. Mater. Sci. 2018, 53, 12807-12815.

(53) Ding, J.-H.; Zhao, H.-R.; Yu, H.-B. A Water-Based Green Approach to Large-Scale Production of Aqueous Compatible Graphene Nanoplatelets. Sci. Rep. 2018, 8, 5567.

(54) Reddy, V.; Satish Babu, K. K. C.; Torati, S. R.; Eom, Y. J.; Trung, T. Q.; Lee, N.-E.; Kim, C. Scalable Production of Water-Dispersible Reduced Graphene Oxide and Its Integration in a Field Effect Transistor. J. Ind. Eng. Chem. 2018, 63, 19-26.

(55) Balasubramanyan, S.; Sasidharan, S.; Poovathinthodiyil, R.; Ramakrishnan, R. M.; Narayanan, B. N. Sucrose-Mediated Mechanical Exfoliation of Graphite: A Green Method for the Large Scale Production of Graphene and Its Application in Catalytic Reduction of 4-Nitrophenol. New J. Chem. 2017, 41, 11969-11978.

(56) González, V. J.; Rodríguez, A. M.; León, V.; Frontiñán-Rubio, J.; Fierro, J. L. G.; Durán-Prado, M.; Muñoz-García, A. B.; Pavone, M.; Vázquez, E. Sweet Graphene: Exfoliation of Graphite and Preparation of Glucose-Graphene Cocrystals through Mechanochemical Treatments. Green Chem. 2018, 20, 3581-3592.

(57) Zhang, X.; Wang, L.; Lu, Q.; Kaplan, D. L. Mass Production of Biocompatible Graphene Using Silk Nanofibers. ACS Appl. Mater. Interfaces 2018, 10, 22924-22931.

(58) Kim, H.-R.; Lee, S.-H.; Lee, K.-H. Scalable Production of Large Single-Layered Graphenes by Microwave Exfoliation in Deionized Water. Carbon 2018, 134, 431-438.

(59) George, G.; Sisupal, S. B.; Tomy, T.; Kumaran, A.; Vadivelu, P.; Suvekbala, V.; Sivaram, S.; Ragupathy, L. Facile, Environmentally Benign and Scalable Approach to Produce Pristine Few Layers Graphene Suitable for Preparing Biocompatible Polymer Nanocomposites. Sci. Rep. 2018, 8, 11228.

(60) González-Domínguez, J. M.; León, V.; Lucío, M. I.; Prato, M.; Vázquez, E. Production of Ready-to-Use Few-Layer Graphene in Aqueous Suspensions. Nat. Protoc. 2018, 13, 495-506.

(61) Yang, S.; Lohe, M. R.; Müllen, K.; Feng, X. New-Generation Graphene from Electrochemical Approaches: Production and Applications. Adv. Mater. 2016, 28, 6213-6221. 
(62) Gao, X.; Lowry, G. V. Progress towards Standardized and Validated Characterizations for Measuring Physicochemical Properties of Manufactured Nanomaterials Relevant to Nano Health and Safety Risks. NanoImpact 2018, 9, 14-30.

(63) Bourdo, S. E.; Al Faouri, R.; Sleezer, R.; Nima, Z. A.; Lafont, A.; Chhetri, B. P.; Benamara, M.; Martin, B.; Salamo, G. J.; Biris, A. S. Physicochemical Characteristics of Pristine and Functionalized Graphene. J. Appl. Toxicol. 2017, 37, 1288-1296.

(64) Li, Y.; Fujita, M.; Boraschi, D. Endotoxin Contamination in Nanomaterials Leads to the Misinterpretation of Immunosafety Results. Front. Immunol. 2017, 8, 472.

(65) Pumera, M.; Ambrosi, A.; Chng, E. L. K. Impurities in Graphenes and Carbon Nanotubes and Their Influence on the Redox Properties. Chem. Sci. 2012, 3, 3347-3355.

(66) Liao, K.-H.; Lin, Y.-S.; Macosko, C. W.; Haynes, C. L. Cytotoxicity of Graphene Oxide and Graphene in Human Erythrocytes and Skin Fibroblasts. ACS Appl. Mater. Interfaces 2011, 3, 2607-2615.

(67) Yang, K.; Wan, J.; Zhang, S.; Zhang, Y.; Lee, S.-T.; Liu, Z. In Vivo Pharmacokinetics, Long-Term Biodistribution, and Toxicology of PEGylated Graphene in Mice. ACS Nano 2011, 5, 516-522.

(68) Duch, M. C.; Budinger, G. R. S.; Liang, Y. T.; Soberanes, S.; Urich, D.; Chiarella, S. E.; Campochiaro, L. A.; Gonzalez, A.; Chandel, N. S.; Hersam, M. C.; Mutlu, G. M. Minimizing Oxidation and Stable Nanoscale Dispersion Improves the Biocompatibility of Graphene in the Lung. Nano Lett. 2011, 11, 5201-5207.

(69) Pinto, A. M.; Moreira, J. A.; Magalhães, F. D.; Gonçalves, I. C. Polymer Surface Adsorption as a Strategy to Improve the Biocompatibility of Graphene Nanoplatelets. Colloids Surf., B 2016, 146, 818-824. (70) Meng, H.; Xia, T.; George, S.; Nel, A. E. A Predictive Toxicological Paradigm for the Safety Assessment of Nanomaterials. ACS Nano 2009, 3, 1620-1627.

(71) Pokhrel, S.; Nel, A. E.; Mädler, L. Custom-Designed Nanomaterial Libraries for Testing Metal Oxide Toxicity. Acc. Chem. Res. 2013, 46 (3), 632-641.

(72) Walkey, C. D.; Olsen, J. B.; Song, F.; Liu, R.; Guo, H.; Olsen, D. W. H.; Cohen, Y.; Emili, A.; Chan, W. C. W. Protein Corona Fingerprinting Predicts the Cellular Interaction of Gold and Silver Nanoparticles. ACS Nano 2014, 8, 2439-2455.

(73) Zhou, H.; Mu, Q.; Gao, N.; Liu, A.; Xing, Y.; Gao, S.; Zhang, Q.; Qu, G.; Chen, Y.; Liu, G.; Zhang, B.; Yan, B. A Nano-Combinatorial Library Strategy for the Discovery of Nanotubes with Reduced ProteinBinding, Cytotoxicity, and Immune Response. Nano Lett. 2008, 8, 859-865.

(74) Li, Y.; Wang, J.; Zhao, F.; Bai, B.; Nie, G.; Nel, A. E.; Zhao, Y. Nanomaterial Libraries and Model Organisms for Rapid High-Content Analysis of Nanosafety. Natl. Sci. Rev. 2018, 5, 365-388.

(75) Docter, D.; Westmeier, D.; Markiewicz, M.; Stolte, S.; Knauer, S. K.; Stauber, R. H. The Nanoparticle Biomolecule Corona: Lessons Learned - Challenge Accepted? Chem. Soc. Rev. 2015, 44, 6094-6121.

(76) Bussy, C.; Kostarelos, K. Culture Media Critically Influence Graphene Oxide Effects on Plasma Membranes. Chem. 2017, 2, 322323.

(77) Chen, R.; Riviere, J. E. Biological Surface Adsorption Index of Nanomaterials: Modelling Surface Interactions of Nanomaterials with Biomolecules. Adv. Exp. Med. Biol. 2017, 947, 207-253.

(78) Graham, U. M.; Jacobs, G.; Yokel, R. A.; Davis, B. H.; Dozier, A. K.; Birch, M. E.; Tseng, M. T.; Oberdörster, G.; Elder, A.; DeLouise, L. From Dose to Response: In Vivo Nanoparticle Processing and Potential Toxicity. Adv. Exp. Med. Biol. 2017, 947, 71-100.

(79) Zhang, D.; Zhang, Z.; Liu, Y.; Chu, M.; Yang, C.; Li, W.; Shao, Y.; Yue, Y.; Xu, R. The Short- and Long-Term Effects of Orally Administered High-Dose Reduced Graphene Oxide Nanosheets on Mouse Behaviors. Biomaterials 2015, 68, 100-113.

(80) Yang, K.; Gong, H.; Shi, X.; Wan, J.; Zhang, Y.; Liu, Z. In Vivo Biodistribution and Toxicology of Functionalized Nano-Graphene Oxide in Mice after Oral and Intraperitoneal Administration. Biomaterials 2013, 34, 2787-2795.

(81) Kucki, M.; Diener, L.; Bohmer, N.; Hirsch, C.; Krug, H. F.; Palermo, V.; Wick, P. Uptake of Label-Free Graphene Oxide by Caco-2
Cells Is Dependent on the Cell Differentiation Status. J. Nanobiotechnol. 2017, 15, 46.

(82) Li, B.; Yang, J.; Huang, Q.; Zhang, Y.; Peng, C.; Zhang, Y.; He, Y.; Shi, J.; Li, W.; Hu, J.; Fan, C. Biodistribution and Pulmonary Toxicity of Intratracheally Instilled Graphene Oxide in Mice. NPG Asia Mater. 2013, 5, e44-e44.

(83) Mao, L.; Hu, M.; Pan, B.; Xie, Y.; Petersen, E. J. Biodistribution and Toxicity of Radio-Labeled Few Layer Graphene in Mice after Intratracheal Instillation. Part. Fibre Toxicol. 2015, 13, 7.

(84) Czarny, B.; Georgin, D.; Berthon, F.; Plastow, G.; Pinault, M.; Patriarche, G.; Thuleau, A.; L'Hermite, M. M.; Taran, F.; Dive, V. Carbon Nanotube Translocation to Distant Organs after Pulmonary Exposure: Insights from in Situ ${ }^{14}$ C-Radiolabeling and Tissue Radioimaging. ACS Nano 2014, 8, 5715-5724.

(85) Sydlik, S. A.; Jhunjhunwala, S.; Webber, M. J.; Anderson, D. G.; Langer, R. In Vivo Compatibility of Graphene Oxide with Differing Oxidation States. ACS Nano 2015, 9, 3866-3874.

(86) Kurantowicz, N.; Strojny, B.; Sawosz, E.; Jaworski, S.; Kutwin, M.; Grodzik, M.; Wierzbicki, M.; Lipińska, L.; Mitura, K.; Chwalibog, A. Biodistribution of a High Dose of Diamond, Graphite, and Graphene Oxide Nanoparticles After Multiple Intraperitoneal Injections in Rats. Nanoscale Res. Lett. 2015, 10, 398.

(87) Syama, S.; Paul, W.; Sabareeswaran, A.; Mohanan, P. V. Raman Spectroscopy for the Detection of Organ Distribution and Clearance of PEGylated Reduced Graphene Oxide and Biological Consequences. Biomaterials 2017, 131, 121-130.

(88) Blanco, E.; Shen, H.; Ferrari, M. Principles of Nanoparticle Design for Overcoming Biological Barriers to Drug Delivery. Nat. Biotechnol. 2015, 33, 941-951.

(89) Qu, G.; Wang, X.; Liu, Q.; Liu, R.; Yin, N.; Ma, J.; Chen, L.; He, J.; Liu, S.; Jiang, G. The Ex Vivo and in Vivo Biological Performances of Graphene Oxide and the Impact of Surfactant on Graphene Oxide's Biocompatibility. J. Environ. Sci. 2013, 25, 873-881.

(90) Wen, K.-P.; Chen, Y.-C.; Chuang, C.-H.; Chang, H.-Y.; Lee, C.Y.; Tai, N.-H. Accumulation and Toxicity of Intravenously-Injected Functionalized Graphene Oxide in Mice. J. Appl. Toxicol. 2015, 35, 1211-1218.

(91) Girish, C. M.; Sasidharan, A.; Gowd, G. S.; Nair, S.; Koyakutty, M. Confocal Raman Imaging Study Showing Macrophage Mediated Biodegradation of Graphene In Vivo. Adv. Healthcare Mater. 2013, 2, $1489-1500$

(92) Li, B.; Zhang, X.; Yang, J.; Zhang, Y.; Li, W.; Fan, C.; Huang, Q. Influence of Polyethylene Glycol Coating On Biodistribution and Toxicity of Nanoscale Graphene Oxide in Mice after Intravenous Injection. Int. J. Nanomed. 2014, 9, 4697-4707.

(93) Sasidharan, A.; Swaroop, S.; Koduri, C. K.; Girish, C. M.; Chandran, P.; Panchakarla, L. S.; Somasundaram, V. H.; Gowd, G. S.; Nair, S.; Koyakutty, M. Comparative in Vivo Toxicity, Organ Biodistribution and Immune Response of Pristine, Carboxylated and PEGylated Few-Layer Graphene Sheets in Swiss Albino Mice: A Three Month Study. Carbon 2015, 95, 511-524.

(94) Liu, J.-H.; Yang, S.-T.; Wang, H.; Chang, Y.; Cao, A.; Liu, Y. Effect of Size and Dose on the Biodistribution of Graphene Oxide in Mice. Nanomedicine 2012, 7, 1801-1812.

(95) Jasim, D. A.; Ménard-Moyon, C.; Bégin, D.; Bianco, A.; Kostarelos, K. Tissue Distribution and Urinary Excretion of Intravenously Administered Chemically Functionalized Graphene Oxide Sheets. Chem. Sci. 2015, 6, 3952-3964.

(96) Oberdörster, G.; Oberdörster, E.; Oberdörster, J. Nanotoxicology: An Emerging Discipline Evolving from Studies of Ultrafine Particles. Environ. Health Perspect. 2005, 113, 823-839.

(97) Stone, V.; Miller, M. R.; Clift, M. J. D.; Elder, A.; Mills, N. L.; Møller, P.; Schins, R. P. F.; Vogel, U.; Kreyling, W. G.; Alstrup Jensen, K.; Kuhlbusch, T. A. J.; Schwarze, P. E.; Hoet, P.; Pietroiusti, A.; De Vizcaya-Ruiz, A.; Baeza-Squiban, A.; Teixeira, J. P.; Tran, C. L.; Cassee, F. R. Nanomaterials Versus Ambient Ultrafine Particles: An Opportunity to Exchange Toxicology Knowledge. Environ. Health Perspect. 2017, 125, 106002. 
(98) Schreiver, I.; Hesse, B.; Seim, C.; Castillo-Michel, H.; Villanova, J.; Laux, P.; Dreiack, N.; Penning, R.; Tucoulou, R.; Cotte, M.; Luch, A. Synchrotron-Based $\nu$-XRF Mapping and $\mu$-FTIR Microscopy Enable to Look into the Fate and Effects of Tattoo Pigments in Human Skin. Sci. Rep. 2017, 7, 11395.

(99) Fadeel, B.; Pietroiusti, A.; Shvedova, A. A. Adverse Effects of Engineered Nanomaterials : Exposure, Toxicology, and Impact on Human Health; 2nd ed. Elsevier, Amsterdam, The Netherlands, 2017.

(100) Bhattacharya, K.; Andón, F. T.; El-Sayed, R.; Fadeel, B. Mechanisms of Carbon Nanotube-Induced Toxicity: Focus on Pulmonary Inflammation. Adv. Drug Delivery Rev. 2013, 65, 20872097.

(101) Orecchioni, M.; Ménard-Moyon, C.; Delogu, L. G.; Bianco, A. Graphene and the Immune System: Challenges and Potentiality. Adv. Drug Delivery Rev. 2016, 105, 163-175.

(102) Russier, J.; Treossi, E.; Scarsi, A.; Perrozzi, F.; Dumortier, H.; Ottaviano, L.; Meneghetti, M.; Palermo, V.; Bianco, A. Evidencing the Mask Effect of Graphene Oxide: A Comparative Study on Primary Human and Murine Phagocytic Cells. Nanoscale 2013, 5, 1123411247.

(103) Yue, H.; Wei, W.; Yue, Z.; Wang, B.; Luo, N.; Gao, Y.; Ma, D.; Ma, G.; Su, Z. The Role of the Lateral Dimension of Graphene Oxide in the Regulation of Cellular Responses. Biomaterials 2012, 33, 40134021.

(104) Ma, J.; Liu, R.; Wang, X.; Liu, Q.; Chen, Y.; Valle, R. P.; Zuo, Y. Y.; Xia, T.; Liu, S. Crucial Role of Lateral Size for Graphene Oxide in Activating Macrophages and Stimulating Pro-Inflammatory Responses in Cells and Animals. ACS Nano 2015, 9, 10498-10515.

(105) Palomäki, J.; Välimäki, E.; Sund, J.; Vippola, M.; Clausen, P. A.; Jensen, K. A.; Savolainen, K.; Matikainen, S.; Alenius, H. Long, Needlelike Carbon Nanotubes and Asbestos Activate the NLRP3 Inflammasome through a Similar Mechanism. ACS Nano 2011, 5, 6861-6870.

(106) Wang, X.; Duch, M. C.; Mansukhani, N.; Ji, Z.; Liao, Y.-P.; Wang, M.; Zhang, H.; Sun, B.; Chang, C. H.; Li, R.; Lin, S.; Meng, H.; Xia, T.; Hersam, M. C.; Nel, A. E. Use of a Pro-Fibrogenic MechanismBased Predictive Toxicological Approach for Tiered Testing and Decision Analysis of Carbonaceous Nanomaterials. ACS Nano 2015, 9, 3032-3043.

(107) Sun, B.; Wang, X.; Ji, Z.; Wang, M.; Liao, Y.-P.; Chang, C. H.; Li, R.; Zhang, H.; Nel, A. E.; Xia, T. NADPH Oxidase-Dependent NLRP3 Inflammasome Activation and Its Important Role in Lung Fibrosis by Multiwalled Carbon Nanotubes. Small 2015, 11, 2087-2097.

(108) Yang, M.; Flavin, K.; Kopf, I.; Radics, G.; Hearnden, C. H. A.; McManus, G. J.; Moran, B.; Villalta-Cerdas, A.; Echegoyen, L. A.; Giordani, S.; Lavelle, E. C. Functionalization of Carbon Nanoparticles Modulates Inflammatory Cell Recruitment and NLRP3 Inflammasome Activation. Small 2013, 9, 4194-4206.

(109) Andón, F. T.; Mukherjee, S. P.; Gessner, I.; Wortmann, L.; Xiao, L.; Hultenby, K.; Shvedova, A. A.; Mathur, S.; Fadeel, B. Hollow Carbon Spheres Trigger Inflammasome-Dependent IL-1 $\beta$ Secretion in Macrophages. Carbon 2017, 113, 243-251.

(110) De Gregorio, E.; Tritto, E.; Rappuoli, R. Alum Adjuvanticity: Unraveling a Century Old Mystery. Eur. J. Immunol. 2008, 38, 20682071.

(111) Mukherjee, S. P.; Bottini, M.; Fadeel, B. Graphene and the Immune System: A Romance of Many Dimensions. Front. Immunol. 2017, 8, 673.

(112) Li, R.; Guiney, L. M.; Chang, C. H.; Mansukhani, N. D.; Ji, Z.; Wang, X.; Liao, Y.-P.; Jiang, W.; Sun, B.; Hersam, M. C.; Nel, A. E.; Xia, T. Surface Oxidation of Graphene Oxide Determines Membrane Damage, Lipid Peroxidation, and Cytotoxicity in Macrophages in a Pulmonary Toxicity Model. ACS Nano 2018, 12, 1390-1402.

(113) Chen, G.-Y.; Yang, H.-J.; Lu, C.-H.; Chao, Y.-C.; Hwang, S.-M.; Chen, C.-L.; Lo, K.-W.; Sung, L.-Y.; Luo, W.-Y.; Tuan, H.-Y.; Hu, Y. C. Simultaneous Induction of Autophagy and Toll-like Receptor Signaling Pathways by Graphene Oxide. Biomaterials 2012, 33, 6559-6569.

(114) Qu, G.; Liu, S.; Zhang, S.; Wang, L.; Wang, X.; Sun, B.; Yin, N.; Gao, X.; Xia, T.; Chen, J.-J.; Jiang, G.-B. Graphene Oxide Induces Toll- like Receptor 4 (TLR4)-Dependent Necrosis in Macrophages. ACS Nano 2013, 7, 5732-5745.

(115) Mukherjee, S. P.; Bondarenko, O.; Kohonen, P.; Andón, F. T.; Brzicová, T.; Gessner, I.; Mathur, S.; Bottini, M.; Calligari, P.; Stella, L.; Kisin, E.; Shvedova, A.; Autio, R.; Salminen-Mankonen, H.; Lahesmaa, R.; Fadeel, B. Macrophage Sensing of Single-Walled Carbon Nanotubes via Toll-like Receptors. Sci. Rep. 2018, 8, 1115.

(116) Barth, N. D.; Marwick, J. A.; Vendrell, M.; Rossi, A. G.; Dransfield, I. The "Phagocytic Synapse" and Clearance of Apoptotic Cells. Front. Immunol. 2017, 8, 1708.

(117) Sosale, N. G.; Spinler, K. R.; Alvey, C.; Discher, D. E. Macrophage Engulfment of a Cell or Nanoparticle Is Regulated by Unavoidable Opsonization, a Species-Specific 'Marker of Self CD47, and Target Physical Properties. Curr. Opin. Immunol. 2015, 35, 107112 .

(118) Hu, W.; Peng, C.; Lv, M.; Li, X.; Zhang, Y.; Chen, N.; Fan, C.; Huang, Q. Protein Corona-Mediated Mitigation of Cytotoxicity of Graphene Oxide. ACS Nano 2011, 5, 3693-3700.

(119) Chong, Y.; Ge, C.; Yang, Z.; Garate, J. A.; Gu, Z.; Weber, J. K.; Liu, J.; Zhou, R. Reduced Cytotoxicity of Graphene Nanosheets Mediated by Blood-Protein Coating. ACS Nano 2015, 9, 5713-5724.

(120) Xu, M.; Zhu, J.; Wang, F.; Xiong, Y.; Wu, Y.; Wang, Q.; Weng, J.; Zhang, Z.; Chen, W.; Liu, S. Improved In Vitro and In Vivo Biocompatibility of Graphene Oxide through Surface Modification: Poly(Acrylic Acid)-Functionalization Is Superior to PEGylation. ACS Nano 2016, 10, 3267-3281.

(121) Belling, J. N.; Jackman, J. A.; Yorulmaz Avsar, S.; Park, J. H.; Wang, Y.; Potroz, M. G.; Ferhan, A. R.; Weiss, P. S.; Cho, N.-J. Stealth Immune Properties of Graphene Oxide Enabled by Surface-Bound Complement Factor H. ACS Nano 2016, 10, 10161-10172.

(122) Matesanz, M.-C.; Vila, M.; Feito, M.-J.; Linares, J.; Gonçalves, G.; Vallet-Regi, M.; Marques, P.-A. A. P.; Portolés, M.-T. The Effects of Graphene Oxide Nanosheets Localized on F-Actin Filaments on CellCycle Alterations. Biomaterials 2013, 34, 1562-1569.

(123) Feito, M. J.; Vila, M.; Matesanz, M. C.; Linares, J.; Gonçalves, G.; Marques, P. A. A. P.; Vallet-Regí, M.; Rojo, J. M.; Portolés, M. T. In Vitro Evaluation of Graphene Oxide Nanosheets on Immune Function. J. Colloid Interface Sci. 2014, 432, 221-228.

(124) Luo, N.; Ni, D.; Yue, H.; Wei, W.; Ma, G. Surface-Engineered Graphene Navigate Divergent Biological Outcomes toward Macrophages. ACS Appl. Mater. Interfaces 2015, 7, 5239-5247.

(125) Luo, N.; Weber, J. K.; Wang, S.; Luan, B.; Yue, H.; Xi, X.; Du, J.; Yang, Z.; Wei, W.; Zhou, R.; Ma, G. PEGylated Graphene Oxide Elicits Strong Immunological Responses despite Surface Passivation. Nat. Commun. 2017, 8, 14537.

(126) Wiemann, M.; Vennemann, A.; Sauer, U. G.; Wiench, K.; MaHock, L.; Landsiedel, R. An in Vitro Alveolar Macrophage Assay for Predicting the Short-Term Inhalation Toxicity of Nanomaterials. J. Nanobiotechnol. 2016, 14, 16.

(127) Mukherjee, S. P.; Lazzaretto, B.; Hultenby, K.; Newman, L.; Rodrigues, A. F.; Lozano, N.; Kostarelos, K.; Malmberg, P.; Fadeel, B. Graphene Oxide Elicits Membrane Lipid Changes and Neutrophil Extracellular Trap Formation. Chem. 2018, 4, 334-358.

(128) Russier, J.; León, V.; Orecchioni, M.; Hirata, E.; Virdis, P.; Fozza, C.; Sgarrella, F.; Cuniberti, G.; Prato, M.; Vázquez, E.; Bianco, A.; Delogu, L. G. Few-Layer Graphene Kills Selectively Tumor Cells from Myelomonocytic Leukemia Patients. Angew. Chem., Int. Ed. 2017, 56, 3014-3019.

(129) Zhou, H.; Zhao, K.; Li, W.; Yang, N.; Liu, Y.; Chen, C.; Wei, T. The Interactions between Pristine Graphene and Macrophages and the Production of Cytokines/Chemokines via TLR- and NF-KB-Related Signaling Pathways. Biomaterials 2012, 33, 6933-6942.

(130) Li, Y.; Liu, Y.; Fu, Y.; Wei, T.; Le Guyader, L.; Gao, G.; Liu, R. S.; Chang, Y.-Z.; Chen, C. The Triggering of Apoptosis in Macrophages by Pristine Graphene through the MAPK and TGF-Beta Signaling Pathways. Biomaterials 2012, 33, 402-411.

(131) Sasidharan, A.; Panchakarla, L. S.; Sadanandan, A. R.; Ashokan, A.; Chandran, P.; Girish, C. M.; Menon, D.; Nair, S. V.; Rao, C. N. R.; 
Koyakutty, M. Hemocompatibility and Macrophage Response of Pristine and Functionalized Graphene. Small 2012, 8, 1251-1263.

(132) Figarol, A.; Pourchez, J.; Boudard, D.; Forest, V.; Akono, C.; Tulliani, J.-M.; Lecompte, J.-P.; Cottier, M.; Bernache-Assollant, D.; Grosseau, P. In Vitro Toxicity of Carbon Nanotubes, Nano-Graphite and Carbon Black, Similar Impacts of Acid Functionalization. Toxicol. In Vitro 2015, 30, 476-485.

(133) Li, Y.; Yuan, H.; von dem Bussche, A.; Creighton, M.; Hurt, R. H.; Kane, A. B.; Gao, H. Graphene Microsheets Enter Cells through Spontaneous Membrane Penetration at Edge Asperities and Corner Sites. Proc. Natl. Acad. Sci. U. S. A. 2013, 110, 12295-12300.

(134) Orecchioni, M.; Bedognetti, D.; Newman, L.; Fuoco, C.; Spada, F.; Hendrickx, W.; Marincola, F. M.; Sgarrella, F.; Rodrigues, A. F.; Ménard-Moyon, C.; Cesareni, G.; Kostarelos, K.; Bianco, A.; Delogu, L. G. Single-Cell Mass Cytometry and Transcriptome Profiling Reveal the Impact of Graphene on Human Immune Cells. Nat. Commun. 2017, 8, 1109.

(135) Kotchey, G. P.; Allen, B. L.; Vedala, H.; Yanamala, N.; Kapralov, A. A.; Tyurina, Y. Y.; Klein-Seetharaman, J.; Kagan, V. E.; Star, A. The Enzymatic Oxidation of Graphene Oxide. ACS Nano 2011, 5, 20982108.

(136) Zhang, C.; Chen, S.; Alvarez, P. J. J.; Chen, W. Reduced Graphene Oxide Enhances Horseradish Peroxidase Stability by Serving as Radical Scavenger and Redox Mediator. Carbon 2015, 94, 531-538.

(137) Li, Y.; Feng, L.; Shi, X.; Wang, X.; Yang, Y.; Yang, K.; Liu, T.; Yang, G.; Liu, Z. Surface Coating-Dependent Cytotoxicity and Degradation of Graphene Derivatives: Towards the Design of NonToxic, Degradable Nano-Graphene. Small 2014, 10, 1544-1554.

(138) Kurapati, R.; Bonachera, F.; Russier, J.; Sureshbabu, A. R.; Ménard-Moyon, C.; Kostarelos, K.; Bianco, A. Covalent Chemical Functionalization Enhances the Biodegradation of Graphene Oxide. $2 D$ Mater. 2018, 5, 015020.

(139) Kurapati, R.; Russier, J.; Squillaci, M. A.; Treossi, E.; MénardMoyon, C.; Del Rio-Castillo, A. E.; Vazquez, E.; Samorì, P.; Palermo, V.; Bianco, A. Dispersibility-Dependent Biodegradation of Graphene Oxide by Myeloperoxidase. Small 2015, 11, 3985-3994.

(140) Mukherjee, S. P.; Gliga, A. R.; Lazzaretto, B.; Brandner, B.; Fielden, M.; Vogt, C.; Newman, L.; Rodrigues, A. F.; Shao, W.; Fournier, P. M.; Toprak, M. S.; Star, A.; Kostarelos, K.; Bhattacharya, K.; Fadeel, B. Graphene Oxide Is Degraded by Neutrophils and the Degradation Products Are Non-Genotoxic. Nanoscale 2018, 10, 11801188.

(141) Shvedova, A. A.; Kapralov, A. A.; Feng, W. H.; Kisin, E. R.; Murray, A. R.; Mercer, R. R.; St. Croix, C. M.; Lang, M. A.; Watkins, S. C.; Konduru, N. V.; Allen, B. L.; Conroy, J.; Kotchey, G. P.; Mohamed, B. M.; Meade, A. D.; Volkov, Y.; Star, A.; Fadeel, B.; Kagan, V. E. Impaired Clearance and Enhanced Pulmonary Inflammatory/Fibrotic Response to Carbon Nanotubes in Myeloperoxidase-Deficient Mice. PLoS One 2012, 7, e30923.

(142) Kagan, V. E.; Kapralov, A. A.; St. Croix, C. M.; Watkins, S. C.; Kisin, E. R.; Kotchey, G. P.; Balasubramanian, K.; Vlasova, I. I.; Yu, J.; Kim, K.; Seo, W.; Mallampalli, R. K.; Star, A.; Shvedova, A. A. Lung Macrophages "Digest" Carbon Nanotubes Using a Superoxide/ Peroxynitrite Oxidative Pathway. ACS Nano 2014, 8, 5610-5621.

(143) Elgrabli, D.; Dachraoui, W.; Ménard-Moyon, C.; Liu, X. J.; Bégin, D.; Bégin-Colin, S.; Bianco, A.; Gazeau, F.; Alloyeau, D. Carbon Nanotube Degradation in Macrophages: Live Nanoscale Monitoring and Understanding of Biological Pathway. ACS Nano 2015, 9, 1011310124 .

(144) Bai, H.; Jiang, W.; Kotchey, G. P.; Saidi, W. A.; Bythell, B. J.; Jarvis, J. M.; Marshall, A. G.; Robinson, R. A. S.; Star, A. Insight into the Mechanism of Graphene Oxide Degradation via the Photo-Fenton Reaction. J. Phys. Chem. C 2014, 118, 10519-10529.

(145) Newman, L.; Lozano, N.; Zhang, M.; Iijima, S.; Yudasaka, M.; Bussy, C.; Kostarelos, K. Hypochlorite Degrades 2D Graphene Oxide Sheets Faster than 1D Oxidised Carbon Nanotubes and Nanohorns. npj 2D Mater Appl. 2017, 1, 39.
(146) Lalwani, G.; Xing, W.; Sitharaman, B. Enzymatic Degradation of Oxidized and Reduced Graphene Nanoribbons by Lignin Peroxidase. J. Mater. Chem. B 2014, 2, 6354-6362.

(147) Liu, L.; Zhu, C.; Fan, M.; Chen, C.; Huang, Y.; Hao, Q.; Yang, J.; Wang, H.; Sun, D. Oxidation and Degradation of Graphitic Materials by Naphthalene-Degrading Bacteria. Nanoscale 2015, 7, 13619-13628.

(148) Zhang, Y.; Bai, Y.; Jia, J.; Gao, N.; Li, Y.; Zhang, R.; Jiang, G.; Yan, B. Perturbation of Physiological Systems by Nanoparticles. Chem. Soc. Rev. 2014, 43, 3762-3809.

(149) Pelin, M.; Sosa, S.; Prato, M.; Tubaro, A. Occupational Exposure to Graphene Based Nanomaterials: Risk Assessment. Nanoscale 2018, 10, 15894-15903.

(150) Kenry, K.; Loh, K. P.; Lim, C. T. Molecular Interactions of Graphene Oxide with Human Blood Plasma Proteins. Nanoscale 2016, 8, 9425-9441.

(151) Mondal, S.; Thirupathi, R.; Rao, L. P.; Atreya, H. S. Unraveling the Dynamic Nature of Protein-graphene Oxide Interactions. RSC Adv. 2016, 6, 52539-52548.

(152) Eedy, D. J. Carbon-Fibre-Induced Airborne Irritant Contact Dermatitis. Contact Dermatitis 1996, 35, 362-363.

(153) Shvedova, A.; Castranova, V.; Kisin, E.; Schwegler-Berry, D.; Murray, A.; Gandelsman, V.; Maynard, A.; Baron, P. Exposure to Carbon Nanotube Material: Assessment of Nanotube Cytotoxicity Using Human Keratinocyte Cells. J. Toxicol. Environ. Health, Part A 2003, 66, 1909-1926.

(154) Pelin, M.; Fusco, L.; León, V.; Martín, C.; Criado, A.; Sosa, S.; Vázquez, E.; Tubaro, A.; Prato, M. Differential Cytotoxic Effects of Graphene and Graphene Oxide on Skin Keratinocytes. Sci. Rep. 2017, 7, 40572.

(155) Pelin, M.; Fusco, L.; Martín, C.; Sosa, S.; Frontiñán-Rubio, J.; González-Domínguez, J. M.; Durán-Prado, M.; Vázquez, E.; Prato, M.; Tubaro, A. Graphene and Graphene Oxide Induce ROS Production in Human HaCaT Skin Keratinocytes: The Role of Xanthine Oxidase and NADH Dehydrogenase. Nanoscale 2018, 10, 11820-11830.

(156) Erf, G. F.; Falcon, D. M.; Sullivan, K. S.; Bourdo, S. E. T Lymphocytes Dominate Local Leukocyte Infiltration in Response to Intradermal Injection of Functionalized Graphene-Based Nanomaterial. J. Appl. Toxicol. 2017, 37, 1317-1324.

(157) Ou, L.; Song, B.; Liang, H.; Liu, J.; Feng, X.; Deng, B.; Sun, T.; Shao, L. Toxicity of Graphene-Family Nanoparticles: A General Review of the Origins and Mechanisms. Part. Part. Fibre Toxicol. 2016, 13, 57.

(158) Roberts, J. R.; Mercer, R. R.; Stefaniak, A. B.; Seehra, M. S.; Geddam, U. K.; Chaudhuri, I. S.; Kyrlidis, A.; Kodali, V. K.; Sager, T.; Kenyon, A.; Bilgesu, S. A.; Eye, T.; Scabilloni, J. F.; Leonard, S. S.; Fix, N. R.; Schwegler-Berry, D.; Farris, B. Y.; Wolfarth, M. G.; Porter, D. W.; Castranova, V.; et al. Evaluation of Pulmonary and Systemic Toxicity Following Lung Exposure to Graphite Nanoplates: A Member of the Graphene-Based Nanomaterial Family. Part. Part. Fibre Toxicol. 2015, $13,34$.

(159) Schinwald, A.; Murphy, F. A.; Jones, A.; MacNee, W.; Donaldson, K. Graphene-Based Nanoplatelets: A New Risk to the Respiratory System as a Consequence of Their Unusual Aerodynamic Properties. ACS Nano 2012, 6, 736-746.

(160) Schinwald, A.; Murphy, F.; Askounis, A.; Koutsos, V.; Sefiane, K.; Donaldson, K.; Campbell, C. J. Minimal Oxidation and Inflammogenicity of Pristine Graphene with Residence in the Lung. Nanotoxicology 2014, 8, 824-832.

(161) Park, E.-J.; Lee, G.-H.; Han, B. S.; Lee, B.-S.; Lee, S.; Cho, M.H.; Kim, J.-H.; Kim, D.-W. Toxic Response of Graphene Nanoplatelets in Vivo and in Vitro. Arch. Toxicol. 2015, 89, 1557-1568.

(162) Park, E.-J.; Lee, S. J.; Lee, K.; Choi, Y. C.; Lee, B.-S.; Lee, G.-H.; Kim, D.-W. Pulmonary Persistence of Graphene Nanoplatelets May Disturb Physiological and Immunological Homeostasis. J. Appl. Toxicol. 2017, 37, 296-309.

(163) Shin, J. H.; Han, S. G.; Kim, J. K.; Kim, B. W.; Hwang, J. H.; Lee, J. S.; Lee, J. H.; Baek, J. E.; Kim, T. G.; Kim, K. S.; Lee, H. S.; Song, N. W.; Ahn, K.; Yu, I. J. 5-Day Repeated Inhalation and 28-Day PostExposure Study of Graphene. Nanotoxicology 2015, 9, 1023-1031. 
(164) Kim, J. K.; Shin, J. H.; Lee, J. S.; Hwang, J. H.; Lee, J. H.; Baek, J. E.; Kim, T. G.; Kim, B. W.; Kim, J. S.; Lee, G. H.; Ahn, K.; Han, S. G.; Bello, D.; Yu, I. J. 28-Day Inhalation Toxicity of Graphene Nanoplatelets in Sprague-Dawley Rats. Nanotoxicology 2016, 10, 891-901.

(165) Lee, J. K.; Jeong, A. Y.; Bae, J.; Seok, J. H.; Yang, J.-Y.; Roh, H. S.; Jeong, J.; Han, Y.; Jeong, J.; Cho, W.-S. The Role of Surface Functionalization on the Pulmonary Inflammogenicity and Translocation into Mediastinal Lymph Nodes of Graphene Nanoplatelets in Rats. Arch. Toxicol. 2017, 91, 667-676.

(166) Bengtson, S.; Knudsen, K. B.; Kyjovska, Z. O.; Berthing, T.; Skaug, V.; Levin, M.; Koponen, I. K.; Shivayogimath, A.; Booth, T. J.; Alonso, B.; Pesquera, A.; Zurutuza, A.; Thomsen, B. L.; Troelsen, J. T.; Jacobsen, N. R.; Vogel, U. Differences in Inflammation and Acute Phase Response but Similar Genotoxicity in Mice Following Pulmonary Exposure to Graphene Oxide and Reduced Graphene Oxide. PLoS One 2017, 12, e0178355.

(167) Bengtson, S.; Kling, K.; Madsen, A. M.; Noergaard, A. W.; Jacobsen, N. R.; Clausen, P. A.; Alonso, B.; Pesquera, A.; Zurutuza, A.; Ramos, R.; Okuno, H.; Dijon, J.; Wallin, H.; Vogel, U. No Cytotoxicity or Genotoxicity of Graphene and Graphene Oxide in Murine Lung Epithelial FE1 Cells in Vitro. Environ. Mol. Mutagen. 2016, 57, 469482.

(168) Poulsen, S. S.; Jackson, P.; Kling, K.; Knudsen, K. B.; Skaug, V.; Kyjovska, Z. O.; Thomsen, B. L.; Clausen, P. A.; Atluri, R.; Berthing, T.; Bengtson, S.; Wolff, H.; Jensen, K. A.; Wallin, H.; Vogel, U. MultiWalled Carbon Nanotube Physicochemical Properties Predict Pulmonary Inflammation and Genotoxicity. Nanotoxicology 2016, 10, $1263-1275$.

(169) Poulsen, S. S.; Saber, A. T.; Williams, A.; Andersen, O.; Købler, C.; Atluri, R.; Pozzebon, M. E.; Mucelli, S. P.; Simion, M.; Rickerby, D.; Mortensen, A.; Jackson, P.; Kyjovska, Z. O.; Mølhave, K.; Jacobsen, N. R.; Jensen, K. A.; Yauk, C. L.; Wallin, H.; Halappanavar, S.; Vogel, U. MWCNTs of Different Physicochemical Properties Cause Similar Inflammatory Responses, but Differences in Transcriptional and Histological Markers of Fibrosis in Mouse Lungs. Toxicol. Appl. Pharmacol. 2015, 284, 16-32.

(170) Vranic, S.; Rodrigues, A. F.; Buggio, M.; Newman, L.; White, M. R. H.; Spiller, D. G.; Bussy, C.; Kostarelos, K. Live Imaging of LabelFree Graphene Oxide Reveals Critical Factors Causing OxidativeStress-Mediated Cellular Responses. ACS Nano 2018, 12, 1373-1389. (171) Drasler, B.; Kucki, M.; Delhaes, F.; Buerki-Thurnherr, T.; Vanhecke, D.; Korejwo, D.; Chortarea, S.; Barosova, H.; Hirsch, C.; Petri-Fink, A.; Rothen-Rutishauser, B.; Wick, P. Single Exposure to Aerosolized Graphene Oxide and Graphene Nanoplatelets Did Not Initiate an Acute Biological Response in a 3D Human Lung Model. Carbon 2018, 137, 125-135.

(172) Shurin, M. R.; Yanamala, N.; Kisin, E. R.; Tkach, A. V.; Shurin, G. V.; Murray, A. R.; Leonard, H. D.; Reynolds, J. S.; Gutkin, D. W.; Star, A.; Fadeel, B.; Savolainen, K.; Kagan, V. E.; Shvedova, A. A. Graphene Oxide Attenuates Th2-Type Immune Responses, but Augments Airway Remodeling and Hyperresponsiveness in a Murine Model of Asthma. ACS Nano 2014, 8, 5585-5599.

(173) Lee, B.-J.; Kim, B.; Lee, K. Air Pollution Exposure and Cardiovascular Disease. Toxicol. Res. 2014, 30, 71-75.

(174) Du, Y.; Xu, X.; Chu, M.; Guo, Y.; Wang, J. Air Particulate Matter and Cardiovascular Disease: The Epidemiological, Biomedical and Clinical Evidence. J. Thorac. Dis. 2016, 8, E8-E19.

(175) Donaldson, K.; Duffin, R.; Langrish, J. P.; Miller, M. R.; Mills, N. L.; Poland, C. A.; Raftis, J.; Shah, A.; Shaw, C. A.; Newby, D. E. Nanoparticles and the Cardiovascular System: A Critical Review. Nanomedicine 2013, 8, 403-423.

(176) Contreras-Torres, F. F.; Rodríguez-Galván, A.; GuerreroBeltrán, C. E.; Martínez-Lorán, E.; Vázquez-Garza, E.; Ornelas-Soto, N.; García-Rivas, G. Differential Cytotoxicity and Internalization of Graphene Family Nanomaterials in Myocardial Cells. Mater. Sci. Eng., C 2017, 73, 633-642.
(177) Singh, S. K.; Singh, M. K.; Nayak, M. K.; Kumari, S.; Shrivastava, S.; Grácio, J. J. A.; Dash, D. Thrombus Inducing Property of Atomically Thin Graphene Oxide Sheets. ACS Nano 2011, 5, 4987-4996.

(178) Singh, S. K.; Singh, M. K.; Kulkarni, P. P.; Sonkar, V. K.; Grácio, J. J. A.; Dash, D. Amine-Modified Graphene: Thrombo-Protective Safer Alternative to Graphene Oxide for Biomedical Applications. ACS Nano 2012, 6, 2731-2740.

(179) Monasterio, B. G.; Alonso, B.; Sot, J.; García-Arribas, A. B.; GilCartón, D.; Valle, M.; Zurutuza, A.; Goñi, F. M. Coating Graphene Oxide with Lipid Bilayers Greatly Decreases Its Hemolytic Properties. Langmuir 2017, 33, 8181-8191.

(180) Cao, Y.; Li, J.; Liu, F.; Li, X.; Jiang, Q.; Cheng, S.; Gu, Y. Consideration of Interaction between Nanoparticles and Food Components for the Safety Assessment of Nanoparticles Following Oral Exposure: A Review. Environ. Toxicol. Pharmacol. 2016, 46, 206210.

(181) Pietroiusti, A.; Bergamaschi, E.; Campagna, M.; Campagnolo, L.; De Palma, G.; Iavicoli, S.; Leso, V.; Magrini, A.; Miragoli, M.; Pedata, P.; Palombi, L.; Iavicoli, I. The Unrecognized Occupational Relevance of the Interaction between Engineered Nanomaterials and the Gastro-Intestinal Tract: A Consensus Paper from a Multidisciplinary Working Group. Part. Fibre Toxicol. 2017, 14, 47.

(182) Abreu, M. T. Toll-like Receptor Signalling in the Intestinal Epithelium: How Bacterial Recognition Shapes Intestinal Function. Nat. Rev. Immunol. 2010, 10, 131-144.

(183) Mabbott, N. A.; Donaldson, D. S.; Ohno, H.; Williams, I. R.; Mahajan, A. Microfold (M) Cells: Important Immunosurveillance Posts in the Intestinal Epithelium. Mucosal Immunol. 2013, 6, 666-677.

(184) Ensign, L. M.; Cone, R.; Hanes, J. Oral Drug Delivery with Polymeric Nanoparticles: The Gastrointestinal Mucus Barriers. Adv. Drug Delivery Rev. 2012, 64, 557-570.

(185) Sun, H.; Chow, E. C.; Liu, S.; Du, Y.; Pang, K. S. The Caco-2 Cell Monolayer: Usefulness and Limitations. Expert Opin. Drug Metab. Toxicol. 2008, 4, 395-411.

(186) Dorier, M.; Brun, E.; Veronesi, G.; Barreau, F.; Pernet-Gallay, K.; Desvergne, C.; Rabilloud, T.; Carapito, C.; Herlin-Boime, N.; Carrière, M. Impact of Anatase and Rutile Titanium Dioxide Nanoparticles on Uptake Carriers and Efflux Pumps in Caco-2 Gut Epithelial Cells. Nanoscale 2015, 7, 7352-7360.

(187) van der Zande, M.; Undas, A. K.; Kramer, E.; Monopoli, M. P.; Peters, R. J.; Garry, D.; Antunes Fernandes, E. C.; Hendriksen, P. J.; Marvin, H. J. P.; Peijnenburg, A. A.; Bouwmeester, H. Different Responses of Caco-2 and MCF-7 Cells to Silver Nanoparticles Are Based on Highly Similar Mechanisms of Action. Nanotoxicology 2016, 10, 1431-1441.

(188) Vila, L.; García-Rodríguez, A.; Cortés, C.; Marcos, R.; Hernández, A. Assessing the Effects of Silver Nanoparticles on Monolayers of Differentiated Caco-2 Cells, as a Model of Intestinal Barrier. Food Chem. Toxicol. 2018, 116, 1-10.

(189) Susewind, J.; de Souza Carvalho-Wodarz, C.; Repnik, U.; Collnot, E.-M.; Schneider-Daum, N.; Griffiths, G. W.; Lehr, C.-M. A 3D Co-Culture of Three Human Cell Lines to Model the Inflamed Intestinal Mucosa for Safety Testing of Nanomaterials. Nanotoxicology 2016, 10, 53-62.

(190) In, J. G.; Foulke-Abel, J.; Estes, M. K.; Zachos, N. C.; Kovbasnjuk, O.; Donowitz, M. Human Mini-Guts: New Insights into Intestinal Physiology and Host-pathogen Interactions. Nat. Rev. Gastroenterol. Hepatol. 2016, 13, 633-642.

(191) Kim, H. J.; Huh, D.; Hamilton, G.; Ingber, D. E. Human Guton-a-Chip Inhabited by Microbial Flora That Experiences Intestinal Peristalsis-like Motions and Flow. Lab Chip 2012, 12, 2165-2174.

(192) Nguyen, T. H. D.; Lin, M.; Mustapha, A. Toxicity of Graphene Oxide on Intestinal Bacteria and Caco-2 Cells. J. Food Prot. 2015, 78, 996-1002.

(193) Ruiz, O. N.; Fernando, K. A. S.; Wang, B.; Brown, N. A.; Luo, P. G.; McNamara, N. D.; Vangsness, M.; Sun, Y.-P.; Bunker, C. E. Graphene Oxide: A Nonspecific Enhancer of Cellular Growth. ACS Nano 2011, 5, 8100-8107. 
(194) Kucki, M.; Rupper, P.; Sarrieu, C.; Melucci, M.; Treossi, E.; Schwarz, A.; León, V.; Kraegeloh, A.; Flahaut, E.; Vázquez, E.; Palermo, V.; Wick, P. Interaction of Graphene-Related Materials with Human Intestinal Cells: An in Vitro Approach. Nanoscale 2016, 8, 8749-8760.

(195) Guarnieri, D.; Sánchez-Moreno, P.; Del Rio Castillo, A. E.; Bonaccorso, F.; Gatto, F.; Bardi, G.; Martín, C.; Vázquez, E.; Catelani, T.; Sabella, S.; Pompa, P. P. Biotransformation and Biological Interaction of Graphene and Graphene Oxide during Simulated Oral Ingestion. Small 2018, 14, 1800227.

(196) Nicholson, J. K.; Holmes, E.; Kinross, J.; Burcelin, R.; Gibson, G.; Jia, W.; Pettersson, S. Host-Gut Microbiota Metabolic Interactions. Science 2012, 336, 1262-1267.

(197) Chen, H.; Zhao, R.; Wang, B.; Zheng, L.; Ouyang, H.; Wang, H.; Zhou, X.; Zhang, D.; Chai, Z.; Zhao, Y.; Feng, W. Acute Oral Administration of Single-Walled Carbon Nanotubes Increases Intestinal Permeability and Inflammatory Responses: Association with the Changes in Gut Microbiota in Mice. Adv. Healthcare Mater. 2018, 7, 1701313 .

(198) Kreyling, W. G.; Semmler-Behnke, M.; Takenaka, S.; Möller, W. Differences in the Biokinetics of Inhaled Nano- versus MicrometerSized Particles. Acc. Chem. Res. 2013, 46, 714-722.

(199) Hougaard, K. S.; Campagnolo, L.; Fadeel, B.; Gulumian, M.; Kagan, V. E.; Møller, P.; Jacobsen, N. R.; Savolainen, K. M. Developmental Toxicity of Engineered Nanomaterials. Reprod. Dev. Toxicol. 2017, 333-357.

(200) Ema, M.; Kobayashi, N.; Naya, M.; Hanai, S.; Nakanishi, J. Reproductive and Developmental Toxicity Studies of Manufactured Nanomaterials. Reprod. Toxicol. 2010, 30, 343-352.

(201) Liang, S.; Xu, S.; Zhang, D.; He, J.; Chu, M. Reproductive Toxicity of Nanoscale Graphene Oxide in Male Mice. Nanotoxicology 2015, 9, 92-105.

(202) Skovmand, A.; Jacobsen Lauvås, A.; Christensen, P.; Vogel, U.; Sørig Hougaard, K.; Goericke-Pesch, S. Pulmonary Exposure to Carbonaceous Nanomaterials and Sperm Quality. Part. Part. Fibre Toxicol. 2018, 15, 10.

(203) Xu, S.; Zhang, Z.; Chu, M. Long-Term Toxicity of Reduced Graphene Oxide Nanosheets: Effects on Female Mouse Reproductive Ability and Offspring Development. Biomaterials 2015, 54, 188-200.

(204) Chen, Y.; Hu, X.; Sun, J.; Zhou, Q. Specific Nanotoxicity of Graphene Oxide during Zebrafish Embryogenesis. Nanotoxicology 2016, 10, 42-52.

(205) Chwalibog, A.; Wierzbicki, M.; Sawosz, E.; Grodzik, M.; Hotowy, A.; Prasek, M.; Jaworski, S.; Sawosz, F. Carbon Nanoparticles Downregulate Expression of Basic Fibroblast Growth Factor in the Heart during Embryogenesis. Int. J. Nanomed. 2013, 8, 3427-3435.

(206) Muoth, C.; Aengenheister, L.; Kucki, M.; Wick, P.; BuerkiThurnherr, T. Nanoparticle Transport across the Placental Barrier: Pushing the Field Forward! Nanomedicine 2016, 11, 941-957.

(207) Huang, X.; Zhang, F.; Sun, X.; Choi, K.-Y.; Niu, G.; Zhang, G.; Guo, J.; Lee, S.; Chen, X. The Genotype-Dependent Influence of Functionalized Multiwalled Carbon Nanotubes on Fetal Development. Biomaterials 2014, 35, 856-865.

(208) Qi, W.; Bi, J.; Zhang, X.; Wang, J.; Wang, J.; Liu, P.; Li, Z.; Wu, W. Damaging Effects of Multi-Walled Carbon Nanotubes on Pregnant Mice with Different Pregnancy Times. Sci. Rep. 2015, 4, 4352.

(209) Campagnolo, L.; Massimiani, M.; Palmieri, G.; Bernardini, R.; Sacchetti, C.; Bergamaschi, A.; Vecchione, L.; Magrini, A.; Bottini, M.; Pietroiusti, A. Biodistribution and Toxicity of Pegylated Single Wall Carbon Nanotubes in Pregnant Mice. Part. Part. Fibre Toxicol. 2013, $10,21$.

(210) Philbrook, N. A.; Walker, V. K.; Afrooz, A. R. M. N.; Saleh, N. B.; Winn, L. M. Investigating the Effects of Functionalized Carbon Nanotubes on Reproduction and Development in Drosophila Melanogaster and CD-1 Mice. Reprod. Toxicol. 2011, 32, 442-448.

(211) Fu, C.; Liu, T.; Li, L.; Liu, H.; Liang, Q.; Meng, X. Effects of Graphene Oxide on the Development of Offspring Mice in Lactation Period. Biomaterials 2015, 40, 23-31.

(212) Kucki, M.; Aengenheister, L.; Diener, L.; Rippl, A. V.; Vranic, S.; Newman, L.; Vazquez, E.; Kostarelos, K.; Wick, P.; Buerki-Thurnherr,
T. Impact of Graphene Oxide on Human Placental Trophoblast Viability, Functionality and Barrier Integrity. 2D Mater. 2018, 5, 035014.

(213) Mendonça, M. C. P.; Soares, E. S.; de Jesus, M. B.; Ceragioli, H. J.; Ferreira, M. S.; Catharino, R. R.; da Cruz-Höfling, M. A. Reduced Graphene Oxide Induces Transient Blood-brain Barrier Opening: An in Vivo Study. J. Nanobiotechnol. 2015, 13, 78.

(214) Pietroiusti, A.; Massimiani, M.; Fenoglio, I.; Colonna, M.; Valentini, F.; Palleschi, G.; Camaioni, A.; Magrini, A.; Siracusa, G.; Bergamaschi, A.; Sgambato, A.; Campagnolo, L. Low Doses of Pristine and Oxidized Single-Wall Carbon Nanotubes Affect Mammalian Embryonic Development. ACS Nano 2011, 5, 4624-4633.

(215) Schmidt, A.; Morales-Prieto, D. M.; Pastuschek, J.; Fröhlich, K.; Markert, U. R. Only Humans Have Human Placentas: Molecular Differences between Mice and Humans. J. Reprod. Immunol. 2015, 108, $65-71$.

(216) Scaini, D.; Ballerini, L. Nanomaterials at the Neural Interface. Curr. Opin. Neurobiol. 2018, 50, 50-55.

(217) Rauti, R.; Lozano, N.; León, V.; Scaini, D.; Musto, M.; Rago, I.; Ulloa Severino, F. P.; Fabbro, A.; Casalis, L.; Vázquez, E.; Kostarelos, K.; Prato, M.; Ballerini, L. Graphene Oxide Nanosheets Reshape Synaptic Function in Cultured Brain Networks. ACS Nano 2016, 10, $4459-4471$

(218) Bramini, M.; Sacchetti, S.; Armirotti, A.; Rocchi, A.; Vázquez, E.; León Castellanos, V.; Bandiera, T.; Cesca, F.; Benfenati, F. Graphene Oxide Nanosheets Disrupt Lipid Composition, Ca ${ }^{2+}$ Homeostasis, and Synaptic Transmission in Primary Cortical Neurons. ACS Nano 2016, 10, 7154-7171.

(219) High, B.; Cole, A. A.; Chen, X.; Reese, T. S. Electron Microscopic Tomography Reveals Discrete Transcleft Elements at Excitatory and Inhibitory Synapses. Front. Synaptic Neurosci. 2015, 7, 9.

(220) Falchi, A. M.; Sogos, V.; Saba, F.; Piras, M.; Congiu, T.; Piludu, M. Astrocytes Shed Large Membrane Vesicles That Contain Mitochondria, Lipid Droplets and ATP. Histochem. Cell Biol. 2013, $139,221-231$.

(221) Antonyak, M. A.; Cerione, R. A. Microvesicles as Mediators of Intercellular Communication in Cancer. Methods Mol. Biol. 2014, 1165, $147-173$

(222) Gottipati, M. K.; Bekyarova, E.; Brenner, M.; Haddon, R. C.; Parpura, V. Changes in the Morphology and Proliferation of Astrocytes Induced by Two Modalities of Chemically Functionalized SingleWalled Carbon Nanotubes Are Differentially Mediated by Glial Fibrillary Acidic Protein. Nano Lett. 2014, 14, 3720-3727.

(223) Shams, H.; Holt, B. D.; Mahboobi, S. H.; Jahed, Z.; Islam, M. F.; Dahl, K. N.; Mofrad, M. R. K. Actin Reorganization through Dynamic Interactions with Single-Wall Carbon Nanotubes. ACS Nano 2014, 8, 188-197.

(224) Tian, X.; Yang, Z.; Duan, G.; Wu, A.; Gu, Z.; Zhang, L.; Chen, C.; Chai, Z.; Ge, C.; Zhou, R. Graphene Oxide Nanosheets Retard Cellular Migration via Disruption of Actin Cytoskeleton. Small 2017, 13,1602133 .

(225) Tu, Q.; Pang, L.; Chen, Y.; Zhang, Y.; Zhang, R.; Lu, B.; Wang, J. Effects of Surface Charges of Graphene Oxide on Neuronal Outgrowth and Branching. Analyst 2014, 139, 105-115.

(226) Fabbro, A.; Scaini, D.; León, V.; Vázquez, E.; Cellot, G.; Privitera, G.; Lombardi, L.; Torrisi, F.; Tomarchio, F.; Bonaccorso, F.; Bosi, S.; Ferrari, A. C.; Ballerini, L.; Prato, M. Graphene-Based Interfaces Do Not Alter Target Nerve Cells. ACS Nano 2016, 10, 615623.

(227) Kitko, K. E.; Hong, T.; Lazarenko, R. M.; Ying, D.; Xu, Y.-Q.; Zhang, Q. Membrane Cholesterol Mediates the Cellular Effects of Monolayer Graphene Substrates. Nat. Commun. 2018, 9, 796.

(228) Pampaloni, N. P.; Lottner, M.; Giugliano, M.; Matruglio, A.; D’Amico, F.; Prato, M.; Garrido, J. A.; Ballerini, L.; Scaini, D. SingleLayer Graphene Modulates Neuronal Communication and Augments Membrane Ion Currents. Nat. Nanotechnol. 2018, 13, 755-764.

(229) Li, N.; Zhang, X.; Song, Q.; Su, R.; Zhang, Q.; Kong, T.; Liu, L.; Jin, G.; Tang, M.; Cheng, G. The Promotion of Neurite Sprouting and 
Outgrowth of Mouse Hippocampal Cells in Culture by Graphene Substrates. Biomaterials 2011, 32, 9374-9382.

(230) Tang, M.; Song, Q.; Li, N.; Jiang, Z.; Huang, R.; Cheng, G. Enhancement of Electrical Signaling in Neural Networks on Graphene Films. Biomaterials 2013, 34, 6402-6411.

(231) Djilas, M.; Olès, C.; Lorach, H.; Bendali, A.; Dégardin, J.; Dubus, E.; Lissorgues-Bazin, G.; Rousseau, L.; Benosman, R.; Ieng, S.H.; Joucla, S.; Yvert, B.; Bergonzo, P.; Sahel, J.; Picaud, S. ThreeDimensional Electrode Arrays for Retinal Prostheses: Modeling, Geometry Optimization and Experimental Validation. J. Neural Eng. 2011, 8, 046020.

(232) Heim, M.; Rousseau, L.; Reculusa, S.; Urbanova, V.; Mazzocco, C.; Joucla, S.; Bouffier, L.; Vytras, K.; Bartlett, P.; Kuhn, A.; Yvert, B. Combined Macro-/Mesoporous Microelectrode Arrays for Low-Noise Extracellular Recording of Neural Networks. J. Neurophysiol. 2012, 108, $1793-1803$.

(233) Gaffaney, J. D.; Dunning, F. M.; Wang, Z.; Hui, E.; Chapman, E. R. Synaptotagmin C2B Domain Regulates Ca ${ }^{2+}$-Triggered Fusion in Vitro. J. Biol. Chem. 2008, 283, 31763-31775.

(234) Weerth, S. H.; Holtzclaw, L. A.; Russell, J. T. Signaling Proteins in Raft-like Microdomains Are Essential for Ca2+ Wave Propagation in Glial Cells. Cell Calcium 2007, 41, 155-167.

(235) Chiacchiaretta, M.; Bramini, M.; Rocchi, A.; Armirotti, A.; Giordano, E.; Vázquez, E.; Bandiera, T.; Ferroni, S.; Cesca, F.; Benfenati, F. Graphene Oxide Upregulates the Homeostatic Functions of Primary Astrocytes and Modulates Astrocyte-to-Neuron Communication. Nano Lett. 2018, 18, 5827-5838.

(236) Cole, M.; Lindeque, P.; Halsband, C.; Galloway, T. S. Microplastics as Contaminants in the Marine Environment: A Review. Mar. Pollut. Bull. 2011, 62, 2588-2597.

(237) Montagner, A.; Bosi, S.; Tenori, E.; Bidussi, M.; Alshatwi, A. A.; Tretiach, M.; Prato, M.; Syrgiannis, Z. Ecotoxicological Effects of Graphene-Based Materials. 2D Mater. 2017, 4, 012001.

(238) Liu, S.; Zeng, T. H.; Hofmann, M.; Burcombe, E.; Wei, J.; Jiang, R.; Kong, J.; Chen, Y. Antibacterial Activity of Graphite, Graphite Oxide, Graphene Oxide, and Reduced Graphene Oxide: Membrane and Oxidative Stress. ACS Nano 2011, 5, 6971-6980.

(239) Maleki Dizaj, S.; Mennati, A.; Jafari, S.; Khezri, K.; Adibkia, K. Antimicrobial Activity of Carbon-Based Nanoparticles. Adv. Pharm. Bull. 2015, 5, 19-23.

(240) Akhavan, O.; Ghaderi, E. Toxicity of Graphene and Graphene Oxide Nanowalls Against Bacteria. ACS Nano 2010, 4, 5731-5736.

(241) Efremova, L. V.; Vasilchenko, A. S.; Rakov, E. G.; Deryabin, D. G. Toxicity of Graphene Shells, Graphene Oxide, and Graphene Oxide Paper Evaluated with Escherichia Coli Biotests. BioMed Res. Int. 2015, 2015, 869361.

(242) Gurunathan, S.; Woong Han, J.; Abdal Daye, A.; Eppakayala, V.; Kim, J. Oxidative Stress-Mediated Antibacterial Activity of Graphene Oxide and Reduced Graphene Oxide in Pseudomonas Aeruginosa. Int. J. Nanomed. 2012, 7, 5901.

(243) Guo, Z.; Xie, C.; Zhang, P.; Zhang, J.; Wang, G.; He, X.; Ma, Y.; Zhao, B.; Zhang, Z. Toxicity and Transformation of Graphene Oxide and Reduced Graphene Oxide in Bacteria Biofilm. Sci. Total Environ. 2017, 580, 1300-1308.

(244) Combarros, R. G.; Collado, S.; Díaz, M. Toxicity of Graphene Oxide on Growth and Metabolism of Pseudomonas Putida. J. Hazard. Mater. 2016, 310, 246-252.

(245) Tu, Y.; Lv, M.; Xiu, P.; Huynh, T.; Zhang, M.; Castelli, M.; Liu, Z.; Huang, Q.; Fan, C.; Fang, H.; Zhou, R. Destructive Extraction of Phospholipids from Escherichia Coli Membranes by Graphene Nanosheets. Nat. Nanotechnol. 2013, 8, 594-601.

(246) Li, R.; Mansukhani, N. D.; Guiney, L. M.; Ji, Z.; Zhao, Y.; Chang, C. H.; French, C. T.; Miller, J. F.; Hersam, M. C.; Nel, A. E.; Xia, T. Identification and Optimization of Carbon Radicals on Hydrated Graphene Oxide for Ubiquitous Antibacterial Coatings. ACS Nano 2016, 10, 10966-10980.

(247) Lu, X.; Feng, X.; Werber, J. R.; Chu, C.; Zucker, I.; Kim, J.-H.; Osuji, C. O.; Elimelech, M. Enhanced Antibacterial Activity through the Controlled Alignment of Graphene Oxide Nanosheets. Proc. Natl. Acad. Sci. U. S. A. 2017, 114, E9793-E9801.

(248) Pandit, S.; Cao, Z.; Mokkapati, V. R. S. S.; Celauro, E.; Yurgens, A.; Lovmar, M.; Westerlund, F.; Sun, J.; Mijakovic, I. Vertically Aligned Graphene Coating Is Bactericidal and Prevents the Formation of Bacterial Biofilms. Adv. Mater. Interfaces 2018, 5, 1701331.

(249) Navarro, E.; Baun, A.; Behra, R.; Hartmann, N. B.; Filser, J.; Miao, A.-J.; Quigg, A.; Santschi, P. H.; Sigg, L. Environmental Behavior and Ecotoxicity of Engineered Nanoparticles to Algae, Plants, and Fungi. Ecotoxicology 2008, 17, 372-386.

(250) Tang, Y.; Tian, J.; Li, S.; Xue, C.; Xue, Z.; Yin, D.; Yu, S. Combined Effects of Graphene Oxide and $\mathrm{Cd}$ on the Photosynthetic Capacity and Survival of Microcystis Aeruginosa. Sci. Total Environ. 2015, 532, 154-161.

(251) Saiz-Jimenez, C. The Conservation of Subterranean Cultural Heritage; CRC Press: Leiden, The Netherlands, 2014.

(252) Cheng, C.; Li, S.; Thomas, A.; Kotov, N. A.; Haag, R. Functional Graphene Nanomaterials Based Architectures: Biointeractions, Fabrications, and Emerging Biological Applications. Chem. Rev. 2017, 117, $1826-1914$.

(253) Du, S.; Zhang, P.; Zhang, R.; Lu, Q.; Liu, L.; Bao, X.; Liu, H. Reduced Graphene Oxide Induces Cytotoxicity and Inhibits Photosynthetic Performance of the Green Alga Scenedesmus Obliquus. Chemosphere 2016, 164, 499-507.

(254) Zhao, J.; Cao, X.; Wang, Z.; Dai, Y.; Xing, B. Mechanistic Understanding toward the Toxicity of Graphene-Family Materials to Freshwater Algae. Water Res. 2017, 111, 18-27.

(255) Hu, X.; Ouyang, S.; Mu, L.; An, J.; Zhou, Q. Effects of Graphene Oxide and Oxidized Carbon Nanotubes on the Cellular Division, Microstructure, Uptake, Oxidative Stress, and Metabolic Profiles. Environ. Sci. Technol. 2015, 49, 10825-10833.

(256) Nogueira, P. F. M.; Nakabayashi, D.; Zucolotto, V. The Effects of Graphene Oxide on Green Algae Raphidocelis Subcapitata. Aquat. Toxicol. 2015, 166, 29-35.

(257) Pereira, M. M.; Mouton, L.; Yéprémian, C.; Couté, A.; Lo, J.; Marconcini, J. M.; Ladeira, L. O.; Raposo, N. R.; Brandão, H. M.; Brayner, R. Ecotoxicological Effects of Carbon Nanotubes and Cellulose Nanofibers in Chlorella Vulgaris. J. Nanobiotechnol. 2014, 12,15 .

(258) Dubinsky, Z.; Schofield, O. From the Light to the Darkness: Thriving at the Light Extremes in the Oceans. Hydrobiologia 2010, 639, 153-171.

(259) Garacci, M.; Barret, M.; Mouchet, F.; Sarrieu, C.; Lonchambon, P.; Flahaut, E.; Gauthier, L.; Silvestre, J.; Pinelli, E. Few Layer Graphene Sticking by Biofilm of Freshwater Diatom Nitzschia Palea as a Mitigation to Its Ecotoxicity. Carbon 2017, 113, 139-150.

(260) Lüttge, U.; Büdel, B. Resurrection Kinetics of Photosynthesis in Desiccation-Tolerant Terrestrial Green Algae (Chlorophyta) on Tree Bark. Plant Biol. 2010, 12, 437-444.

(261) Holzinger, A.; Karsten, U. Desiccation Stress and Tolerance in Green Algae: Consequences for Ultrastructure, Physiological and Molecular Mechanisms. Front. Plant Sci. 2013, 4, 327.

(262) Montagner, A.; Bosi, S.; Tenori, E.; Bidussi, M.; Alshatwi, A. A.; Tretiach, M.; Prato, M.; Syrgiannis, Z. Ecotoxicological Effects of Graphene-Based Materials. 2D Mater. 2017, 4, 012001.

(263) Wang, Y.; Chang, C. H.; Ji, Z.; Bouchard, D. C.; Nisbet, R. M.; Schimel, J. P.; Gardea-Torresdey, J. L.; Holden, P. A. Agglomeration Determines Effects of Carbonaceous Nanomaterials on Soybean Nodulation, Dinitrogen Fixation Potential, and Growth in Soil. ACS Nano 2017, 11, 5753-5765.

(264) Begum, P.; Fugetsu, B. Induction of Cell Death by Graphene in Arabidopsis Thaliana (Columbia Ecotype) T87 Cell Suspensions. J. Hazard. Mater. 2013, 260, 1032-1041.

(265) Wang, Q.; Zhao, S.; Zhao, Y.; Rui, Q.; Wang, D. Toxicity and Translocation of Graphene Oxide in Arabidopsis Plants under Stress Conditions. RSC Adv. 2014, 4, 60891-60901.

(266) Zhao, S.; Wang, Q.; Zhao, Y.; Rui, Q.; Wang, D. Toxicity and Translocation of Graphene Oxide in Arabidopsis Thaliana. Environ. Toxicol. Pharmacol. 2015, 39, 145-156. 
(267) Chen, L.; Wang, C.; Li, H.; Qu, X.; Yang, S.-T.; Chang, X.-L. Bioaccumulation and Toxicity of ${ }^{13}$ C-Skeleton Labeled Graphene Oxide in Wheat. Environ. Sci. Technol. 2017, 51, 10146-10153.

(268) Liu, S.; Wei, H.; Li, Z.; Li, S.; Yan, H.; He, Y.; Tian, Z. Effects of Graphene on Germination and Seedling Morphology in Rice. J. Nanosci. Nanotechnol. 2015, 15, 2695-2701.

(269) Anjum, N. A.; Singh, N.; Singh, M. K.; Sayeed, I.; Duarte, A. C.; Pereira, E.; Ahmad, I. Single-Bilayer Graphene Oxide Sheet Impacts and Underlying Potential Mechanism Assessment in Germinating Faba Bean (Vicia Faba L.). Sci. Total Environ. 2014, 472, 834-841.

(270) Chichiriccò, G.; Poma, A. Penetration and Toxicity of Nanomaterials in Higher Plants. Nanomaterials 2015, 5, 851-873.

(271) Begum, P.; Ikhtiari, R.; Fugetsu, B. Graphene Phytotoxicity in the Seedling Stage of Cabbage, Tomato, Red Spinach, and Lettuce. Carbon 2011, 49, 3907-3919.

(272) Candotto Carniel, F.; Gorelli, D.; Flahaut, E.; Fortuna, L.; Del Casino, C.; Cai, G.; Nepi, M.; Prato, M.; Tretiach, M. Graphene Oxide Impairs the Pollen Performance of Nicotiana Tabacum and Corylus Avellana Suggesting Potential Negative Effects on the Sexual Reproduction of Seed Plants. Environ. Sci.: Nano 2018, 5, 1608-1617. (273) Zhang, W.; Wang, C.; Li, Z.; Lu, Z.; Li, Y.; Yin, J.-J.; Zhou, Y.-T.; Gao, X.; Fang, Y.; Nie, G.; Zhao, Y. Unraveling Stress-Induced Toxicity Properties of Graphene Oxide and the Underlying Mechanism. Adv. Mater. 2012, 24, 5391-5397.

(274) Jung, S.-K.; Qu, X.; Aleman-Meza, B.; Wang, T.; Riepe, C.; Liu, Z.; Li, Q.; Zhong, W. Multi-Endpoint, High-Throughput Study of Nanomaterial Toxicity in Caenorhabditis Elegans. Environ. Sci. Technol. 2015, 49, 2477-2485.

(275) Zhao, Y.; Wu, Q.; Wang, D. An Epigenetic Signal Encoded Protection Mechanism Is Activated by Graphene Oxide to Inhibit Its Induced Reproductive Toxicity in Caenorhabditis Elegans. Biomaterials 2016, 79, 15-24.

(276) Yang, R.; Ren, M.; Rui, Q.; Wang, D. A Mir-231-Regulated Protection Mechanism against the Toxicity of Graphene Oxide in Nematode Caenorhabditis Elegans. Sci. Rep. 2016, 6, 32214.

(277) Ren, M.; Zhao, L.; Lv, X.; Wang, D. Antimicrobial Proteins in the Response to Graphene Oxide in Caenorhabditis Elegans. Nanotoxicology 2017, 11, 578-590.

(278) Zanni, E.; De Bellis, G.; Bracciale, M. P.; Broggi, A.; Santarelli, M. L.; Sarto, M. S.; Palleschi, C.; Uccelletti, D. Graphite Nanoplatelets and Caenorhabditis Elegans : Insights from an in Vivo Model. Nano Lett. 2012, 12, 2740-2744.

(279) Dziewięcka, M.; Karpeta-Kaczmarek, J.; Augustyniak, M.; Majchrzycki, Ł.; Augustyniak-Jabłokow, M. A. Evaluation of in Vivo Graphene Oxide Toxicity for Acheta Domesticus in Relation to Nanomaterial Purity and Time Passed from the Exposure. J. Hazard. Mater. 2016, 305, 30-40.

(280) Pretti, C.; Oliva, M.; Pietro, R. Di; Monni, G.; Cevasco, G.; Chiellini, F.; Pomelli, C.; Chiappe, C. Ecotoxicity of Pristine Graphene to Marine Organisms. Ecotoxicol. Environ. Saf. 2014, 101, 138-145.

(281) Guo, X.; Dong, S.; Petersen, E. J.; Gao, S.; Huang, Q.; Mao, L. Biological Uptake and Depuration of Radio-Labeled Graphene by Daphnia Magna. Environ. Sci. Technol. 2013, 47, 12524-12531.

(282) Souza, J. P.; Venturini, F. P.; Santos, F.; Zucolotto, V. Chronic Toxicity in Ceriodaphnia Dubia Induced by Graphene Oxide. Chemosphere 2018, 190, 218-224.

(283) De Marchi, L.; Neto, V.; Pretti, C.; Figueira, E.; Brambilla, L.; Rodriguez-Douton, M. J.; Rossella, F.; Tommasini, M.; Furtado, C.; Soares, A. M. V. M.; Freitas, R. Physiological and Biochemical Impacts of Graphene Oxide in Polychaetes: The Case of Diopatra Neapolitana. Comp. Biochem. Physiol., Part C: Toxicol. Pharmacol. 2017, 193, 50-60.

(284) Zhang, P.; Selck, H.; Tangaa, S. R.; Pang, C.; Zhao, B. Bioaccumulation and Effects of Sediment-Associated Gold- and Graphene Oxide Nanoparticles on Tubifex Tubifex. J. Environ. Sci. 2017, 51, 138-145.

(285) Hu, C.; Wang, Q.; Zhao, H.; Wang, L.; Guo, S.; Li, X. Ecotoxicological Effects of Graphene Oxide on the Protozoan Euglena Gracilis. Chemosphere 2015, 128, 184-190.
(286) Mesarič, T.; Sepčič, K.; Piazza, V.; Gambardella, C.; Garaventa, F.; Drobne, D.; Faimali, M. Effects of Nano Carbon Black and SingleLayer Graphene Oxide on Settlement, Survival and Swimming Behaviour of Amphibalanus Amphitrite Larvae. Chem. Ecol. 2013, 29, 643-652.

(287) Grillo, R.; Rosa, A. H.; Fraceto, L. F. Engineered Nanoparticles and Organic Matter: A Review of the State-of-the-Art. Chemosphere 2015, 119, 608-619.

(288) Castro, V. L.; Clemente, Z.; Jonsson, C.; Silva, M.; Vallim, J. H.; de Medeiros, A. M. Z.; Martinez, D. S. T. Nanoecotoxicity Assessment of Graphene Oxide and Its Relationship with Humic Acid. Environ. Toxicol. Chem. 2018, 37, 1998-2012.

(289) Maes, H. M.; Stibany, F.; Giefers, S.; Daniels, B.; Deutschmann, B.; Baumgartner, W.; Schäffer, A. Accumulation and Distribution of Multiwalled Carbon Nanotubes in Zebrafish (Danio Rerio). Environ. Sci. Technol. 2014, 48, 12256-12264.

(290) Liu, X. T.; Mu, X. Y.; Wu, X. L.; Meng, L. X.; Guan, W. B.; Ma, Y. Q.; Sun, H.; Wang, C. J.; Li, X. F. Toxicity of Multi-Walled Carbon Nanotubes, Graphene Oxide, and Reduced Graphene Oxide to Zebrafish Embryos. Biomed. Environ. Sci. 2014, 27, 676-683.

(291) Chen, L.; Hu, P.; Zhang, L.; Huang, S.; Luo, L.; Huang, C. Toxicity of Graphene Oxide and Multi-Walled Carbon Nanotubes against Human Cells and Zebrafish. Sci. China: Chem. 2012, 55, 22092216.

(292) Souza, J. P.; Baretta, J. F.; Santos, F.; Paino, I. M. M.; Zucolotto, V. Toxicological Effects of Graphene Oxide on Adult Zebrafish (Danio Rerio). Aquat. Toxicol. 2017, 186, 11-18.

(293) Zhang, X.; Zhou, Q.; Zou, W.; Hu, X. Molecular Mechanisms of Developmental Toxicity Induced by Graphene Oxide at Predicted Environmental Concentrations. Environ. Sci. Technol. 2017, 51, 78617871.

(294) Mouchet, F.; Landois, P.; Flahaut, E.; Pinelli, E.; Gauthier, L. Assessment of the Potential in Vivo Ecotoxicity of Double-Walled Carbon Nanotubes (DWNTs) in Water, Using the Amphibian Ambystoma Mexicanum. Nanotoxicology 2007, 1, 149-156.

(295) Mouchet, F.; Landois, P.; Sarremejean, E.; Bernard, G.; Puech, P.; Pinelli, E.; Flahaut, E.; Gauthier, L. Characterisation and in Vivo Ecotoxicity Evaluation of Double-Wall Carbon Nanotubes in Larvae of the Amphibian Xenopus Laevis. Aquat. Toxicol. 2008, 87, 127-137.

(296) Mouchet, F.; Landois, P.; Puech, P.; Pinelli, E.; Flahaut, E.; Gauthier, L. Carbon Nanotube Ecotoxicity in Amphibians: Assessment of Multiwalled Carbon Nanotubes and Comparison with DoubleWalled Carbon Nanotubes. Nanomedicine 2010, 5, 963-974.

(297) Muzi, L.; Mouchet, F.; Cadarsi, S.; Janowska, I.; Russier, J.; Ménard-Moyon, C.; Risuleo, G.; Soula, B.; Galibert, A.-M.; Flahaut, E.; Pinelli, E.; Gauthier, L.; Bianco, A. Examining the Impact of MultiLayer Graphene Using Cellular and Amphibian Models. 2D Mater. 2016, 3, 025009.

(298) Mottier, A.; Mouchet, F.; Laplanche, C.; Cadarsi, S.; Lagier, L.; Arnault, J.-C.; Girard, H. A.; León, V.; Vázquez, E.; Sarrieu, C.; Pinelli, E.; Gauthier, L.; Flahaut, E. Surface Area of Carbon Nanoparticles: A Dose Metric for a More Realistic Ecotoxicological Assessment. Nano Lett. 2016, 16, 3514-3518.

(299) Lagier, L.; Mouchet, F.; Laplanche, C.; Mottier, A.; Cadarsi, S.; Evariste, L.; Sarrieu, C.; Lonchambon, P.; Pinelli, E.; Flahaut, E.; Gauthier, L. Surface Area of Carbon-Based Nanoparticles Prevails on Dispersion for Growth Inhibition in Amphibians. Carbon 2017, 119, $72-81$.

(300) Auffan, M.; Tella, M.; Santaella, C.; Brousset, L.; Paillès, C.; Barakat, M.; Espinasse, B.; Artells, E.; Issartel, J.; Masion, A.; Rose, J.; Wiesner, M. R.; Achouak, W.; Thiéry, A.; Bottero, J.-Y. An Adaptable Mesocosm Platform for Performing Integrated Assessments of Nanomaterial Risk in Complex Environmental Systems. Sci. Rep. 2015, 4, 5608.

(301) Bour, A.; Mouchet, F.; Silvestre, J.; Gauthier, L.; Pinelli, E. Environmentally Relevant Approaches to Assess Nanoparticles Ecotoxicity: A Review. J. Hazard. Mater. 2015, 283, 764-777.

(302) Mottier, A.; Mouchet, F.; Pinelli, E.; Gauthier, L.; Flahaut, E. Environmental Impact of Engineered Carbon Nanoparticles: From 
Releases to Effects on the Aquatic Biota. Curr. Opin. Biotechnol. 2017, $46,1-6$.

(303) Bour, A.; Mouchet, F.; Verneuil, L.; Evariste, L.; Silvestre, J.; Pinelli, E.; Gauthier, L. Toxicity of $\mathrm{CeO} 2$ Nanoparticles at Different Trophic Levels - Effects on Diatoms, Chironomids and Amphibians. Chemosphere 2015, 120, 230-236.

(304) Bour, A.; Mouchet, F.; Cadarsi, S.; Silvestre, J.; Verneuil, L.; Baqué, D.; Chauvet, E.; Bonzom, J.-M.; Pagnout, C.; Clivot, H.; Fourquaux, I.; Tella, M.; Auffan, M.; Gauthier, L.; Pinelli, E. Toxicity of $\mathrm{CeO} 2$ Nanoparticles on a Freshwater Experimental Trophic Chain: A Study in Environmentally Relevant Conditions through the Use of Mesocosms. Nanotoxicology 2016, 10, 245-255.

(305) Hu, X.; Kang, J.; Lu, K.; Zhou, R.; Mu, L.; Zhou, Q. Graphene Oxide Amplifies the Phytotoxicity of Arsenic in Wheat. Sci. Rep. 2015, 4, 6122 .

(306) Wang, D.; Wang, G.; Zhang, G.; Xu, X.; Yang, F. Using Graphene Oxide to Enhance the Activity of Anammox Bacteria for Nitrogen Removal. Bioresour. Technol. 2013, 131, 527-530.

(307) Du, J.; Hu, X.; Zhou, Q. Graphene Oxide Regulates the Bacterial Community and Exhibits Property Changes in Soil. RSC Adv. 2015, 5, 27009-27017.

(308) Xiong, T.; Yuan, X.; Wang, H.; Leng, L.; Li, H.; Wu, Z.; Jiang, L.; $\mathrm{Xu}, \mathrm{R}$.; Zeng, G. Implication of Graphene Oxide in Cd-Contaminated Soil: A Case Study of Bacterial Communities. J. Environ. Manage. 2018, 205, 99-106.

(309) Ghosal, D.; Ghosh, S.; Dutta, T. K.; Ahn, Y. Current State of Knowledge in Microbial Degradation of Polycyclic Aromatic Hydrocarbons (PAHs): A Review. Front. Microbiol. 2016, 7, 1369.

(310) Xu, Y.; Zhou, N.-Y. Microbial Remediation of AromaticsContaminated Soil. Front. Environ. Sci. Eng. 2017, 11, 1.

(311) Tortella, G. R.; Diez, M. C.; Durán, N. Fungal Diversity and Use in Decomposition of Environmental Pollutants. Crit. Rev. Microbiol. 2005, 31, 197-212.

(312) Schreiner, K. M.; Filley, T. R.; Blanchette, R. A.; Bowen, B. B.; Bolskar, R. D.; Hockaday, W. C.; Masiello, C. A.; Raebiger, J. W. WhiteRot Basidiomycete-Mediated Decomposition of $\mathrm{C}_{60}$ Fullerol. Environ. Sci. Technol. 2009, 43, 3162-3168.

(313) Xie, J.; Ming, Z.; Li, H.; Yang, H.; Yu, B.; Wu, R.; Liu, X.; Bai, Y.; Yang, S.-T. Toxicity of Graphene Oxide to White Rot Fungus Phanerochaete Chrysosporium. Chemosphere 2016, 151, 324-331.

(314) Yang, H.; Feng, S.; Ma, Q.; Ming, Z.; Bai, Y.; Chen, L.; Yang, S.T. Influence of Reduced Graphene Oxide on the Growth, Structure and Decomposition Activity of White-Rot Fungus Phanerochaete Chrysosporium. RSC Adv. 2018, 8, 5026-5033.

(315) Ankley, G. T.; Bennett, R. S.; Erickson, R. J.; Hoff, D. J.; Hornung, M. W.; Johnson, R. D.; Mount, D. R.; Nichols, J. W.; Russom, C. L.; Schmieder, P. K.; Serrrano, J. A.; Tietge, J. E.; Villeneuve, D. L. Adverse Outcome Pathways: A Conceptual Framework to Support Ecotoxicology Research and Risk Assessment. Environ. Toxicol. Chem. 2010, 29, 730-741.

(316) Leist, M.; Ghallab, A.; Graepel, R.; Marchan, R.; Hassan, R.; Bennekou, S. H.; Limonciel, A.; Vinken, M.; Schildknecht, S.; Waldmann, T.; Danen, E.; van Ravenzwaay, B.; Kamp, H.; Gardner, I.; Godoy, P.; Bois, F. Y.; Braeuning, A.; Reif, R.; Oesch, F.; Drasdo, D.; et al. Adverse Outcome Pathways: Opportunities, Limitations and Open Questions. Arch. Toxicol. 2017, 91, 3477-3505.

(317) Groso, A.; Petri-Fink, A.; Magrez, A.; Riediker, M.; Meyer, T. Management of Nanomaterials Safety in Research Environment. Part. Part. Fibre Toxicol. 2010, 7, 40.

(318) Imhof, C.; Clark, K.; Meyer, T.; Schmid, K.; Riediker, M. Research and Development-where People Are Exposed to Nanomaterials. J. Occup. Health 2015, 57, 179-188.

(319) Spinazzè, A.; Cattaneo, A.; Campagnolo, D.; Bollati, V.; Bertazzi, P. A.; Cavallo, D. M. Engineered Nanomaterials Exposure in the Production of Graphene. Aerosol Sci. Technol. 2016, 50, 812-821. (320) Lee, J. H.; Han, J. H.; Kim, J. H.; Kim, B.; Bello, D.; Kim, J. K.; Lee, G. H.; Sohn, E. K.; Lee, K.; Ahn, K.; Faustman, E. M.; Yu, I. J. Exposure Monitoring of Graphene Nanoplatelets Manufacturing Workplaces. Inhalation Toxicol. 2016, 28, 281-291.
(321) Lo, L.-M.; Hammond, D.; Bartholomew, I.; Almaguer, D.; Heitbrink, W.; Topmiller, J. Engineering Controls for Nano-Scale Graphene Platelets During Manufacturing and Handling Processes. Department of Health and Human Services Centers for Disease Control and Prevention National Institute for Occupational Safety and Health, 2011.

(322) Heitbrink, W. A.; Lo, L.-M.; Dunn, K. H. Exposure Controls for Nanomaterials at Three Manufacturing Sites. J. Occup. Environ. Hyg. 2015, 12, 16-28.

(323) Rebitzer, G.; Ekvall, T.; Frischknecht, R.; Hunkeler, D.; Norris, G.; Rydberg, T.; Schmidt, W.-P.; Suh, S.; Weidema, B. P.; Pennington, D. W. Life Cycle Assessment: Part 1: Framework, Goal and Scope Definition, Inventory Analysis, and Applications. Environ. Int. 2004, 30, 701-720.

(324) Arvidsson, R.; Kushnir, D.; Molander, S.; Sandén, B. A. Energy and Resource Use Assessment of Graphene as a Substitute for Indium Tin Oxide in Transparent Electrodes. J. Cleaner Prod. 2016, 132, 289297.

(325) Arvidsson, R.; Molander, S. Prospective Life Cycle Assessment of Epitaxial Graphene Production at Different Manufacturing Scales and Maturity. J. Ind. Ecol. 2017, 21, 1153-1164.

(326) Pizza, A.; Metz, R.; Hassanzadeh, M.; Bantignies, J.-L. Life Cycle Assessment of Nanocomposites Made of Thermally Conductive Graphite Nanoplatelets. Int. J. Life Cycle Assess. 2014, 19, 1226-1237.

(327) Arvidsson, R.; Kushnir, D.; Sandén, B. A.; Molander, S. Prospective Life Cycle Assessment of Graphene Production by Ultrasonication and Chemical Reduction. Environ. Sci. Technol. 2014, 48, 4529-4536.

(328) Rosenbaum, R. K.; Bachmann, T. M.; Gold, L. S.; Huijbregts, M. A. J.; Jolliet, O.; Juraske, R.; Koehler, A.; Larsen, H. F.; MacLeod, M.; Margni, M.; McKone, T. E.; Payet, J.; Schuhmacher, M.; van de Meent, D.; Hauschild, M. Z. USEtox-the UNEP-SETAC Toxicity Model: Recommended Characterisation Factors for Human Toxicity and Freshwater Ecotoxicity in Life Cycle Impact Assessment. Int. J. Life Cycle Assess. 2008, 13, 532-546.

(329) Salieri, B.; Righi, S.; Pasteris, A.; Olsen, S. I. Freshwater Ecotoxicity Characterisation Factor for Metal Oxide Nanoparticles: A Case Study on Titanium Dioxide Nanoparticle. Sci. Total Environ. 2015, 505, 494-502.

(330) Ettrup, K.; Kounina, A.; Hansen, S. F.; Meesters, J. A. J.; Vea, E. B.; Laurent, A. Development of Comparative Toxicity Potentials of TiO ${ }_{2}$ Nanoparticles for Use in Life Cycle Assessment. Environ. Sci. Technol. 2017, 51, 4027-4037.

(331) Meesters, J. A. J.; Koelmans, A. A.; Quik, J. T. K.; Hendriks, A. J.; van de Meent, D. Multimedia Modeling of Engineered Nanoparticles with SimpleBox4nano: Model Definition and Evaluation. Environ. Sci. Technol. 2014, 48, 5726-5736.

(332) Salieri, B.; Turner, D. A.; Nowack, B.; Hischier, R. Life Cycle Assessment of Manufactured Nanomaterials: Where Are We? NanoImpact 2018, 10, 108-120.

(333) Kostarelos, K. Translating Graphene and 2D Materials into Medicine. Nat. Rev. Mater. 2016, 1, 16084.

(334) Valsami-Jones, E.; Lynch, I. How Safe Are Nanomaterials? Science 2015, 350, 388-389.

(335) Yang, K.; Li, Y.; Tan, X.; Peng, R.; Liu, Z. Behavior and Toxicity of Graphene and Its Functionalized Derivatives in Biological Systems. Small 2013, 9, 1492-1503.

(336) Seabra, A. B.; Paula, A. J.; de Lima, R.; Alves, O. L.; Durán, N. Nanotoxicity of Graphene and Graphene Oxide. Chem. Res. Toxicol. 2014, 27, 159-168.

(337) Costa, P. M.; Fadeel, B. Emerging Systems Biology Approaches in Nanotoxicology: Towards a Mechanism-Based Understanding of Nanomaterial Hazard and Risk. Toxicol. Appl. Pharmacol. 2016, 299, $101-111$.

(338) Nel, A.; Xia, T.; Meng, H.; Wang, X.; Lin, S.; Ji, Z.; Zhang, H. Nanomaterial Toxicity Testing in the 21 st Century: Use of a Predictive Toxicological Approach and High-Throughput Screening. Acc. Chem. Res. 2013, 46, 607-621. 
(339) Fadeel, B.; Farcal, L.; Hardy, B.; Vázquez-Campos, S.; Hristozov, D.; Marcomini, A.; Lynch, I.; Valsami-Jones, E.; Alenius, H.; Savolainen, K. Advanced Tools for the Safety Assessment of Nanomaterials. Nat. Nanotechnol. 2018, 13, 537-543.

(340) Brockmeier, E. K.; Hodges, G.; Hutchinson, T. H.; Butler, E.; Hecker, M.; Tollefsen, K. E.; Garcia-Reyero, N.; Kille, P.; Becker, D.; Chipman, K.; Colbourne, J.; Collette, T. W.; Cossins, A.; Cronin, M.; Graystock, P.; Gutsell, S.; Knapen, D.; Katsiadaki, I.; Lange, A.; Marshall, S.; et al. The Role of Omics in the Application of Adverse Outcome Pathways for Chemical Risk Assessment. Toxicol. Sci. 2017, $158,252-262$.

(341) Wittwehr, C.; Aladjov, H.; Ankley, G.; Byrne, H. J.; de Knecht, J.; Heinzle, E.; Klambauer, G.; Landesmann, B.; Luijten, M.; MacKay, C.; Maxwell, G.; Meek, M. E.; Paini, A.; Perkins, E.; Sobanski, T.; Villeneuve, D.; Waters, K. M.; Whelan, M. How Adverse Outcome Pathways Can Aid the Development and Use of Computational Prediction Models for Regulatory Toxicology. Toxicol. Sci. 2017, 155, 326-336.

(342) Worth, A.; Aschberger, K.; Bofill, D. A.; Bessems, J.; Gerloff, K.; Graepel, R.; Joossens, E.; Lamon, L.; Palosaari, T.; Richarz, A. Evaluation of the Availability and Applicability of Computational Approaches in the Safety Assessment of Nanomaterials Final Report of the Nanocomput Project. JRC Sci. Technol. Report. 2017, DOI: $10.2760 / 248139$.

(343) Burello, E. Review of (Q)SAR Models for Regulatory Assessment of Nanomaterials Risks. NanoImpact 2017, 8, 48-58.

(344) Grosse, Y.; Loomis, D.; Guyton, K. Z.; Lauby-Secretan, B.; El Ghissassi, F.; Bouvard, V.; Benbrahim-Tallaa, L.; Guha, N.; Scoccianti, C.; Mattock, H.; Straif, K. Carcinogenicity of Fluoro-Edenite, Silicon Carbide Fibres and Whiskers, and Carbon Nanotubes. Lancet Oncol. 2014, 15, 1427-1428.

(345) Kurapati, R.; Mukherjee, S. P.; Martín, C.; Bepete, G.; Vázquez, E.; Pénicaud, A.; Fadeel, B.; Bianco, A. Degradation of Single-Layer and Few-Layer Graphene by Neutrophil Myeloperoxidase. Angew. Chem., Int. Ed. 2018, 57, 11722-11727.

(346) Kurapati, R.; Kostarelos, K.; Prato, M.; Bianco, A. Biomedical Uses for 2D Materials Beyond Graphene: Current Advances and Challenges Ahead. Adv. Mater. 2016, 28, 6052-6074.

(347) Guiney, L. M.; Wang, X.; Xia, T.; Nel, A. E.; Hersam, M. C. Assessing and Mitigating the Hazard Potential of Two-Dimensional Materials. ACS Nano 2018, 12, 6360-6377.

(348) Faria, M.; Björnmalm, M.; Thurecht, K. J.; Kent, S. J.; Parton, R. G.; Kavallaris, M.; Johnston, A. P. R.; Gooding, J. J.; Corrie, S. R.; Boyd, B. J.; Thordarson, P.; Whittaker, A. K.; Stevens, M. M.; Prestidge, C. A.; Porter, C. J. H.; Parak, W. J.; Davis, T. P.; Crampin, E. J.; Caruso, F. Minimum Information Reporting in Bio-nano Experimental Literature. Nat. Nanotechnol. 2018, 13, 777-785. 